\title{
C-F bond activation under transition-metal-free conditions
}

\author{
Han-Jun $\mathrm{Ai}^{1,2 \dagger}$, Xingxing $\mathrm{Ma}^{3,4 \dagger}$, Qiuling Song ${ }^{3,4^{*}}$ \& Xiao-Feng $\mathrm{Wu}^{1,2^{*}}$ \\ ${ }^{1}$ Leibniz-Institut fur Katalyse e.V. an der Universität Rostock, Rostock 18059, Germany; \\ ${ }^{2}$ Dalian National Laboratory for Clean Energy, Dalian Institute of Chemical Physics, Chinese Academy of Sciences, Dalian 116023, China; \\ ${ }^{3}$ Key Laboratory of Molecule Synthesis and Function Discovery, Fujian Province University, College of Chemistry, Fuzhou University, \\ Fuzhou 350108, China; \\ ${ }^{4}$ Institute of Next Generation Matter Transformation, College of Material Sciences \& Engineering, Huaqiao University, \\ Xiamen 361021, China
}

Received April 25, 2021; accepted June 4, 2021; published online August 30, 2021

\begin{abstract}
The unique properties of fluorine-containing organic compounds make fluorine substitution attractive for the development of pharmaceuticals and various specialty materials, which have inspired the evolution of diverse $\mathrm{C}-\mathrm{F}$ bond activation techniques. Although many advances have been made in functionalizations of activated $\mathrm{C}-\mathrm{F}$ bonds utilizing transition metal complexes, there are fewer approaches available for nonactivated $\mathrm{C}-\mathrm{F}$ bonds due to the difficulty in oxidative addition of transition metals to the inert C-F bonds. In this regard, using Lewis acid to abstract the fluoride and light/radical initiator to generate the radical intermediate have emerged as powerful tools for activating those inert C-F bonds. Meanwhile, these transition-metal-free processes are greener, economical, and for the pharmaceutical industry, without heavy metal residues. This review provides an overview of recent $\mathrm{C}-\mathrm{F}$ bond activations and functionalizations under transition-metal-free conditions. The key mechanisms involved are demonstrated and discussed in detail. Finally, a brief discussion on the existing limitations of this field and our perspective are presented.
\end{abstract}

transition-metal-free, fluorinated compounds, $\mathrm{C}-\mathrm{F}$ activation, coupling

Citation: Ai HJ, Ma X, Song Q, Wu XF. C-F bond activation under transition-metal-free conditions. Sci China Chem, 2021, 64: 1630-1659, https://doi.org/ $10.1007 / \mathrm{s} 11426-021-1040-2$

\section{Introduction}

Fluorinated organic compounds are distinguished in diverse fields such as materials science, polymer chemistry, pesticides and pharmaceuticals [1-6]. This is mainly due to the unique nature of $\mathrm{C}-\mathrm{F}$ bonds, which can dramatically change the reactivity and properties of parent molecules [7,8]. However, these features also make the $\mathrm{C}-\mathrm{F}$ bond the strongest $\mathrm{C}$-heteroatom bond linkages in nature [8]. Beyond that, the kinetic issues are also responsible for the inertness of the $\mathrm{C}-\mathrm{F}$ bond, and thus in most cases, fluorine is not a good

$\dagger$ These authors contributed equally to this work.

*Corresponding authors (email: qsong@hqu.edu.cn; xiao-feng.wu@catalysis.de) leaving group [9]. The above reasons lead to these molecules not easily modified, extraordinarily long-lived and potentially toxic. The study of processes that allow fluorine to be substituted by other atoms or functional groups is an academic challenge and has great promise for applications.

Realizing the importance of $\mathrm{C}-\mathrm{F}$ bond activation, chemists have put great efforts into $\mathrm{C}-\mathrm{F}$ bond conversion and functionalization to explore milder reaction conditions and more efficient transformations. This review will mainly discuss transition-metal-free $\mathrm{C}-\mathrm{F}$ bond activations to form the $\mathrm{C}-\mathrm{H}$, $\mathrm{C}-\mathrm{C}, \mathrm{C}-\mathrm{Si}, \mathrm{C}-\mathrm{N}, \mathrm{C}-\mathrm{B}, \mathrm{C}-\mathrm{P}, \mathrm{C}-\mathrm{O}, \mathrm{C}-\mathrm{S}$ bonds and so on. The numerous examples of transition-metal-catalyzed $\mathrm{C}-\mathrm{F}$ bond activations have already been well documented and reviewed [10-20]. 
In general, the most obvious advantage of the transitionmetal-free $\mathrm{C}-\mathrm{F}$ bond activation methods is the cheapness and greenness, and in the field of drug development, without heavy metal residues. What is more, the approach of transition metals for $\mathrm{C}-\mathrm{F}$ bond cleavage typically underwent oxidative addition of the $\mathrm{C}-\mathrm{F}$ bond to an electron-rich metal center [21] or involves the homolytic splitting of $\mathrm{C}-\mathrm{F}$ bonds via a single-electron transfer process [22]. However, the substrate scope is mainly limited to activated aromatic and vinylic $\mathrm{C}\left(\mathrm{sp}^{2}\right)-\mathrm{F}$ bond, or/and strong nucleophilic reagents; [10-20], the $\mathrm{C}-\mathrm{F}$ bond transformations of nonactivated aliphatic fluorides have received less attention [23,24]. Contingent on the activation mode, three strategies might be distinguished for the $\mathrm{C}-\mathrm{F}$ bond cleavage under transitionmetal-free conditions: (1) using Lewis acid to directly abstract the fluoride; (2) in the presence of strong bases or/and strong nucleophilic reagents, fluorine are nucleophilically substituted; (3) through $\mathrm{C}-\mathrm{F}$ bond homolysis, $\mathrm{C}-\mathrm{F}$ bond activation via a radical-based pathway has emerged as a promising and conceptually novel strategy in recent years. Under transition-metal-free conditions, the $\mathrm{C}-\mathrm{F}$ bondbreaking event proceeds gently and selectively with light or radical initiator.

It is necessary to understand the bond dissociation energies (BDE) of different $\mathrm{C}-\mathrm{F}$ bonds to study the activation of $\mathrm{C}-\mathrm{F}$ bonds. As shown in Scheme 1 [25-27], the greater the degree of unsaturation or the more substituents, the higher the BDEs (Scheme 1, left). The BDE of the C-F bond of alkyl fluorides is also related to the amount of fluorine on the carbon. For example, the BDE of $\mathrm{CF}_{4}$ and $\mathrm{CH}_{3} \mathrm{~F}$ are 547 and $460 \mathrm{~kJ} / \mathrm{mol}$, respectively (Scheme 1, middle). In general, the BDEs of $\mathrm{C}\left(\mathrm{sp}^{2}\right)-\mathrm{F}$ bonds are higher than those of $\mathrm{C}\left(\mathrm{sp}^{3}\right)-\mathrm{F}$ bonds. However, the BDEs of $\mathrm{C}-\mathrm{F}$ bonds of polyfluoroarenes are much lower (Scheme 1, right). This review will cover the activation of most of those $\mathrm{C}-\mathrm{F}$ bonds, and the key mechanisms involved will be demonstrated and discussed in detail. The literature in this review is mostly from 2015 to the present and includes some selected examples during 2000 2015. A small amount of earlier literature is selected to demonstrate the historical advances of the related topics.

\section{Hydrodefluorination}

Catalytic hydrodefluorination (HDF) is an exciting process with great application potential. Not only because it offers a strategy for converting cheaply available perfluorinated or polyfluorinated hydrocarbon into more valuable fluorinated compounds, but also because it is considered as a solution to address the super long-lived greenhouse gas: chlorofluorocarbons, hydrofluorocarbons, and perfluorocarbons (for example, the atmospheric lifetime of $\mathrm{CF}_{4}$ can exceed 50,000 years) [28]. Therefore, the current trend in HDF is

\begin{tabular}{|c|c|c|c|c|c|}
\hline C-F bonds & BDEs (kJ / mol) & C-F bonds & BDEs (kJ / mol) & C-F bonds & BDEs (kJ / mol) \\
\hline$\sim^{\mathrm{F}}$ & 405 & $\mathrm{CF}_{3}=\mathrm{F}$ & 547 & & \\
\hline $\mathrm{Et}=\mathrm{F}$ & 467 & $\mathrm{CHF}_{2}=\mathrm{F}$ & 534 & $\mathrm{C}_{6} \mathrm{~F}_{5}=\mathrm{F}$ & 485 \\
\hline${ }^{i} \operatorname{Pr}=F$ & 483 & $\mathrm{CH}_{2} \mathrm{~F}=\mathrm{F}$ & 496 & $\mathrm{C}_{6} \mathrm{H}_{5-\mathrm{x}} \mathrm{F}_{\mathrm{x}}=\mathrm{F}$ & - \\
\hline $\mathrm{Cy}=\mathrm{F}$ & 491 & $\mathrm{CH}_{3}=\mathrm{F}$ & 460 & & \\
\hline${ }^{t} \mathrm{Bu}=\mathrm{F}$ & 496 & $\mathrm{PhCF}_{2}=\mathrm{F}$ & 481 & $\mathrm{C}_{6} \mathrm{H}_{5}=\mathrm{F}$ & 526 \\
\hline & 518 & $\mathrm{PhCHF}=\mathrm{F}$ & - & & \\
\hline$\equiv-F$ & 521 & $\mathrm{PhCH}_{2}=\mathrm{F}$ & 413 & & \\
\hline
\end{tabular}

Scheme 1 Overview of the BDEs of various C-F bonds (color online).

towards the use of milder solutions for the decomposition of stable $\mathrm{C}-\mathrm{F}$ bonds and preparing more valuable products from readily available polyfluorinated compounds.

\subsection{Silicon electrophiles}

Under transition-metal-free conditions to break the high activation barrier of the $\mathrm{C}-\mathrm{F}$ bond, an exceptionally potent Lewis acid with high fluoride affinity is normally required. The formation of more stable main-group element-fluorine bonds is thought to be the key driving force for this transition. The earliest work on the use of Lewis acid to seize fluoride from fluoroalkane was reported by Krause and Lampe [29] in 1977. They observed the redistribution of $\mathrm{Si}-$ $\mathrm{H} / \mathrm{C}-\mathrm{F}$ by mass spectrometry in the gas phase upon collision of $\mathrm{SiH}_{3}{ }^{+}$with $\mathrm{CF}_{4}$. Subsequently, the Ozerov's group [30-32] further demonstrated that silicon cations $\left(\mathrm{R}_{3} \mathrm{Si}^{+}\right)$, as the extremely strong Lewis acids, could readily abstract fluorides from fluorocarbons by reporting the catalytic $\mathrm{HDF}$ of $\mathrm{C}$ $\left(\mathrm{sp}^{3}\right)-\mathrm{F}$ bonds at room temperature. Initially, they employed $\mathrm{Et}_{3} \mathrm{Si}^{+}\left[\mathrm{B}\left(\mathrm{C}_{6} \mathrm{~F}_{5}\right)_{4}\right]^{-}$as catalyst and $\mathrm{Et}_{3} \mathrm{SiH}$ as the $\mathrm{H}$ source (Scheme 2). Under this catalytic system, silylium ions reacted with almost any $\mathrm{C}\left(\mathrm{sp}^{3}\right)-\mathrm{F}$ bond [30], while $\mathrm{C}\left(\mathrm{sp}^{2}\right)-\mathrm{F}$ bond was retained. They speculated that the inertness of 3 might be due to the relative difficulty of generating perfluoroalkyl cations. Moreover, the catalytic efficiency is highly dependent on the nature of the counter anion, and the authors identified that although $\left[\mathrm{B}\left(\mathrm{C}_{6} \mathrm{~F}_{5}\right)_{4}\right]^{-}$is experimentally compatible with triethylsilylium, it is ostensibly not resistant to carbocations and high levels of Brønsted acidity; however, decomposition of $\left[\mathrm{B}\left(\mathrm{C}_{6} \mathrm{~F}_{5}\right)_{4}\right]^{-}$by silylium ions limited the turnover numbers (TONs).

The choice of halogenated monocarboranes [33], which are compatible with the highest levels of Brønsted Lewis [34], as the supporting anions dramatically improved the longevity of the catalysis (Scheme 3 ) and allowed the TON of the catalytic HDF up to 2,700 [31]. The high efficiency of the process offers the possibility of large-scale restoration applications. In addition to this, although the metathesis of the $\mathrm{Si}-\mathrm{H} / \mathrm{C}-\mathrm{F}$ is thermodynamically favorable, this article illustrates that maintaining the Lewis acidity in the reaction system is the key to the overall catalytic HDF reaction. 


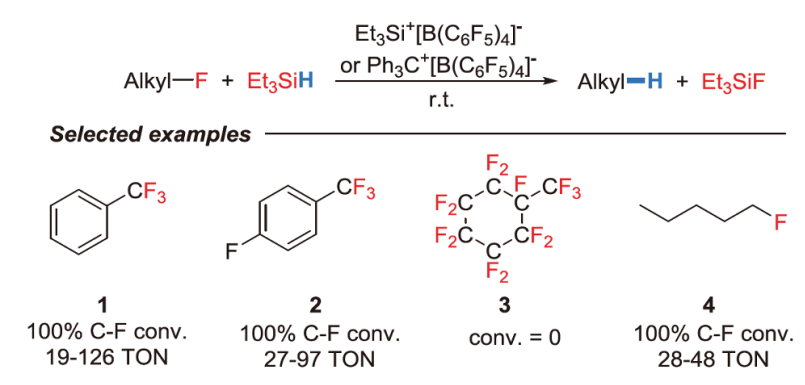

Scheme 2 The first catalytic HDF of $\mathrm{C}\left(\mathrm{sp}^{3}\right)-\mathrm{F}$ bonds (color online).
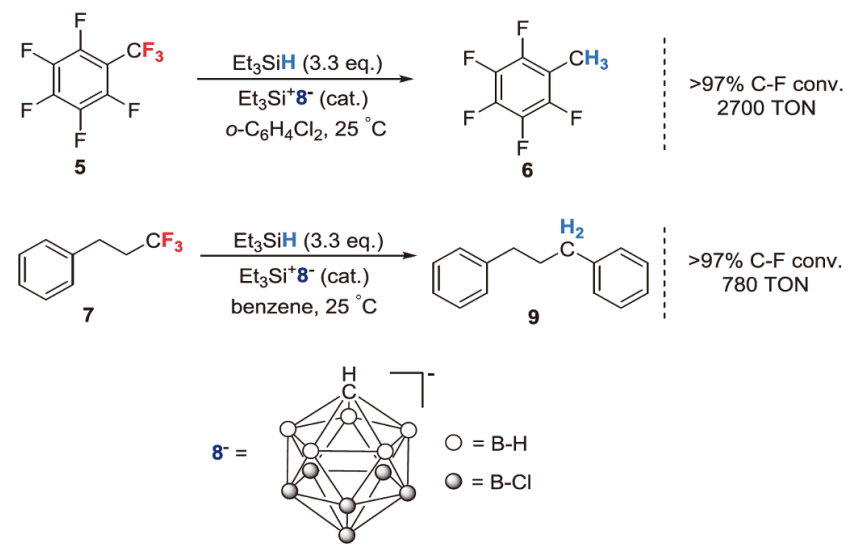

Scheme 3 Silylium-carborane catalyzed HDF (color online).

Similarly, Müller and co-workers [35] designed a hydridebridged disilyl cation $\mathbf{1 0}^{+}$for HDF (Scheme 4). Trifluoromethyl benzene 1 could be converted entirely to toluene under mild conditions when a catalytic amount of $\mathbf{1 0}^{+}\left[\mathrm{B}\left(\mathrm{C}_{6} \mathrm{~F}_{5}\right)_{4}\right]^{-}$was used. The success of this catalytic cycle is based on the fact that by treatment with an excess of $\mathrm{Et}_{3} \mathrm{SiH}, \mathbf{1 2}^{+}$can be converted to $\mathbf{1 0}^{+}$.

Subsequently, Stephan and co-workers [36] reported F/H exchange between alkyl fluorides and $\mathrm{Et}_{3} \mathrm{SiH}$ employing the commercially available strong organoborane Lewis acid $\mathrm{B}\left(\mathrm{C}_{6} \mathrm{~F}_{5}\right)_{3}[37,38]$ as the catalyst (Scheme 5). Through $\eta^{1}$ coordination to activate silane $[39,40]$, primary $(\mathbf{1 3}, \mathbf{1 4})$, secondary (15) and tertiary (16) alkyl fluorides can be successfully converted into the corresponding alkanes. Notably, due to the lower reactivity of the $\mathrm{B}\left(\mathrm{C}_{6} \mathrm{~F}_{5}\right)_{3} / \mathrm{Et}_{3} \mathrm{SiH}$ system compared with silylium ions, the $\mathrm{CF}_{3}$ group in $\mathbf{1 7}$ did not react, resulting in selective HDF of the fluoromethoxy fragment. The slower reaction in this case is attributed to the presence of the heteroatom, which often prevents the reaction by their nucleophilicity and the potential of donor-acceptor interactions.

Their subsequent research [41] shows that besides common Lewis acids, electrophilic $\mathrm{P}^{\mathrm{III}}$ compounds can also achieve catalytic HDF reactions (Scheme 6). Quantitative reduction of $\mathrm{C}-\mathrm{F}$ bonds was observed for primary (18),

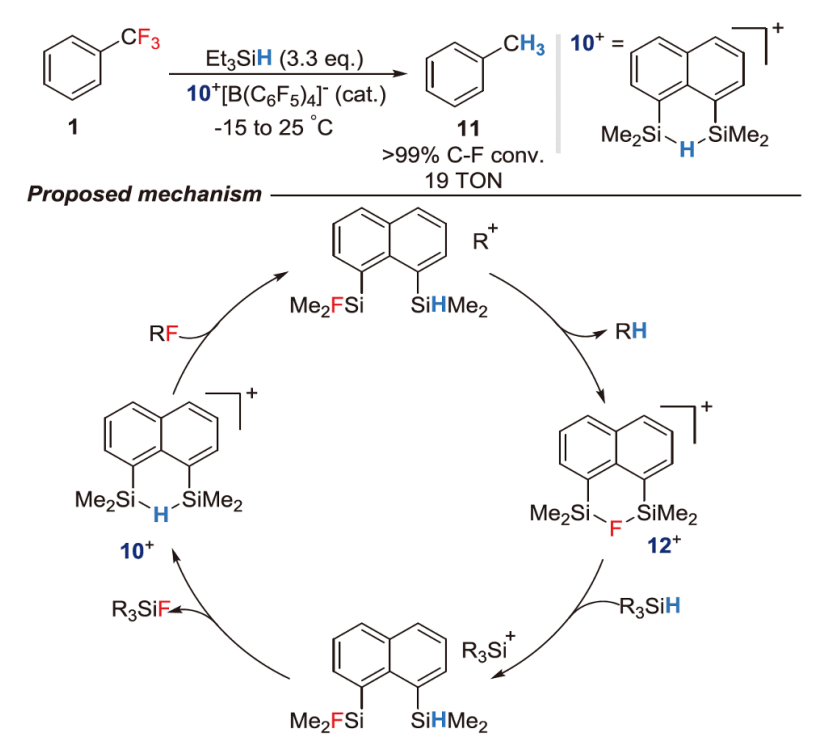

Scheme 4 Hydride-bridged disilyl cation for catalytic HDF (color online).

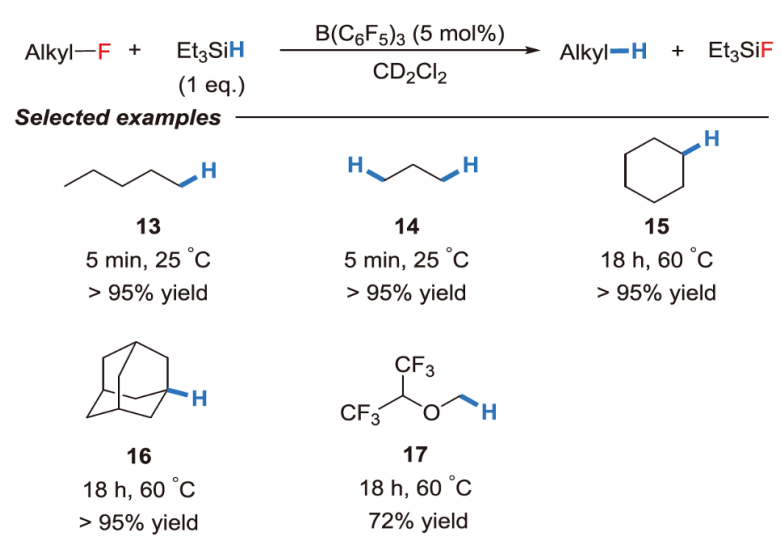

Scheme $5 \quad B\left(C_{6} \mathrm{~F}_{5}\right)_{3}$-catalyzed HDF of alkyl fluorides (color online).

secondary (19), or tertiary fluoroalkanes $(\mathbf{2 0})$. The $\mathrm{CF}_{3}$ group in $\mathrm{PhCF}_{3}$ (21) was converted to $\mathrm{CH}_{3}$ group. The $\mathrm{CHF}_{2}$ or $\mathrm{CH}_{2} \mathrm{~F}$ containing intermediates were not detected even when a limited amount of $\mathrm{Et}_{3} \mathrm{SiH}$ was used. That is probably because the $\mathrm{BDE}$ of $\mathrm{CHF}_{2}$ and $\mathrm{CH}_{2} \mathrm{~F}$ are lower than $\mathrm{CF}_{3}$ (vide supra).

Recently, Ogoshi's group [42] reported a pentacoordinated hydrosilicates (tetrabutylammonium difluorotriphenylsilicate, TBAT) catalyzed HDF reaction (Scheme 7). Employing hydrosilane as the $\mathrm{H}$ source, polyfluoroarenes were converted into the HDF or twofold-HDF products such as $\mathbf{2 2}$, 23, 24 and 25 with high regioselectivity. The higher the electron density, the lower the reactivity and regioselectivity of the substrates. (26). Notably, functional groups are generally incompatible with strong Lewis acids, such as nitro group (27), cyano groups (28), ester (29), amides (30), chlororide (31) and pyridine (32) are tolerated in this cata- 

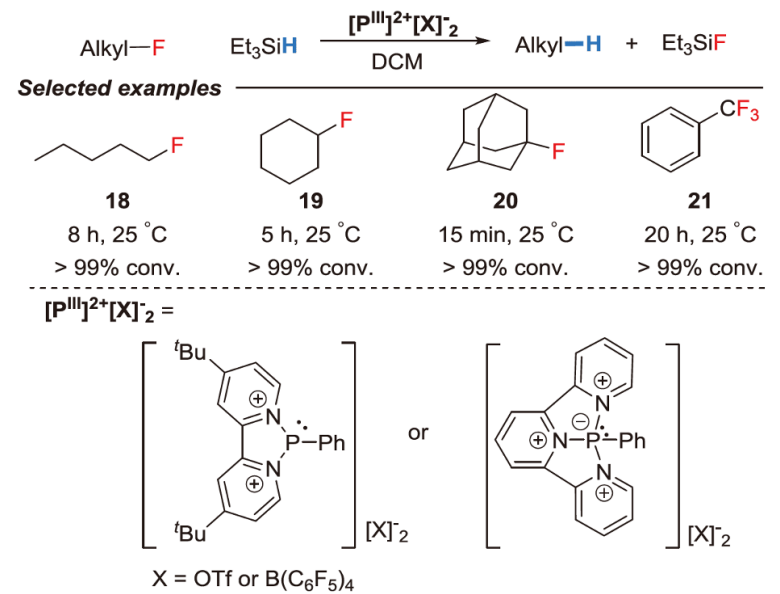

Scheme $6 \mathrm{P}^{\mathrm{III}}$ Lewis acid catalyzed HDF of alkyl fluorides (color online).

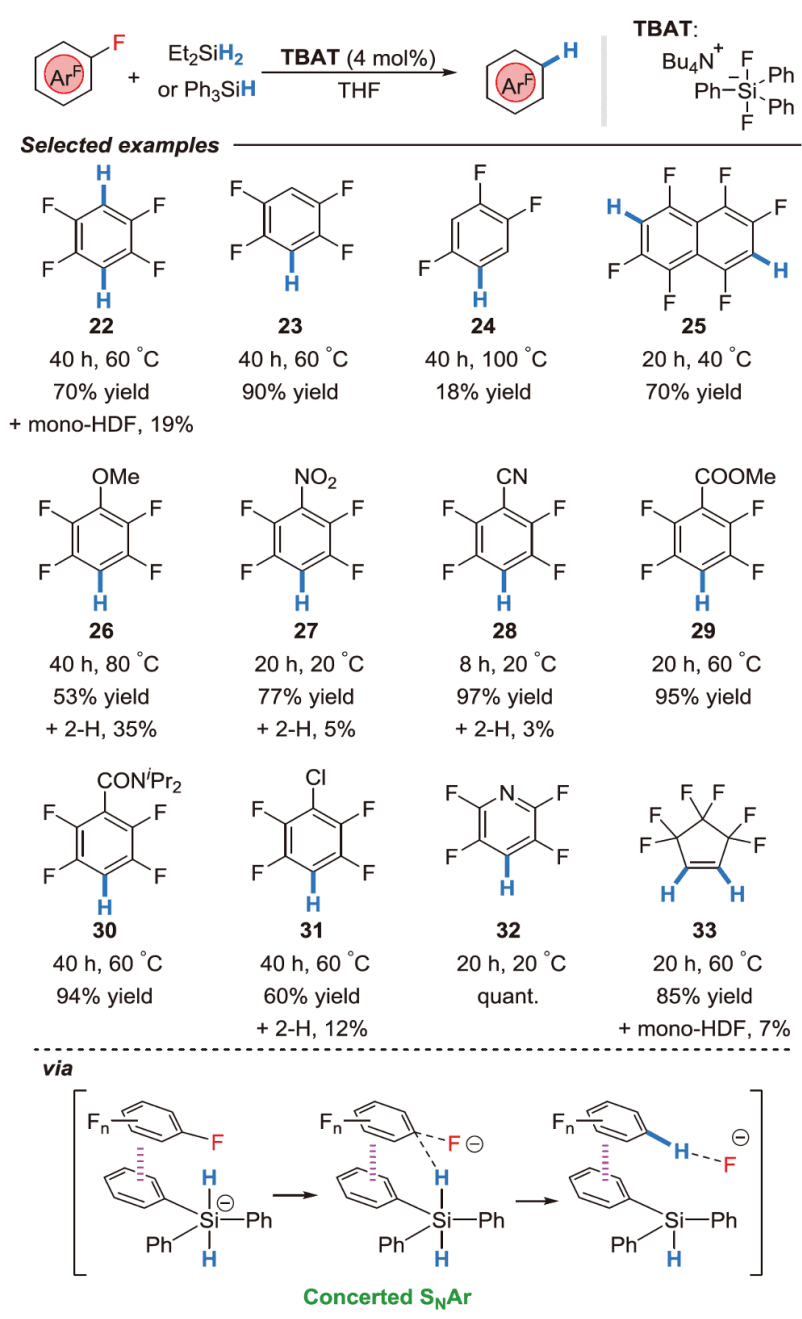

Scheme 7 TBAT-catalyzed HDF by concerted $\mathrm{S}_{\mathrm{N}} \mathrm{Ar}$ (color online).

lytic system. Furthermore, octafluorocyclopentene could be transformed, whereby only the HDF of the alkenyl fluoride proceeded to afford 33. Unlike the method which utilizes silylium cations as strong Lewis acids to abstract the fluoride (vide supra), this transformation underwent a pentacoordinated hydrosilicates-catalyzed concerted nucleophilic aromatic substitution $\left(\mathrm{CS}_{\mathrm{N}} \mathrm{Ar}\right)$ process. The key to this success is that the eliminated fluoride can be regenerated to the hydrosilicate and complete the catalytic cycle.

The heterogeneous catalytic $\mathrm{C}-\mathrm{F}$ activation of fluoromethanes was achieved by Kemnitz and Braun's group $[43,44]$ with nanoscopic aluminium chlorofluoride (ACF) as the catalyst (Scheme 8). ACF is an amorphous chlorinecontaining aluminum fluoride $\mathrm{AlCl} \mathrm{F}_{3}-x(x \approx 0.05-0.3)$ and is considered to be the strongest solid Lewis acid with acidity comparable to that of $\mathrm{SbF}_{5}$ [45-47]. In a glass ampule under mild reaction conditions, $\mathrm{CH}_{3} \mathrm{~F}(\mathbf{3 4})$ yielded $\mathrm{Et}_{3} \mathrm{SiF}_{\mathrm{F}}$ and $\mathrm{CH}_{4}$ with a TON up to 400 . For $\mathrm{CH}_{2} \mathrm{~F}_{2}(35)$, the TON can be increased to 112. The HDF of $\mathrm{CHF}_{3}(\mathbf{3 6})$ was also achieved, albeit at elevated temperatures and prolonged reaction time. A surface-bound silylium-ion-like species is considered as the crucial intermediate in the $\mathrm{C}-\mathrm{F}$ bond cleavage step for this heterogeneous catalytic process.

\subsection{Aluminum electrophiles}

In addition to silylium ions, aluminium ions can also be applied to catalytic HDF reactions. For example, Rosenthal, Krossing and co-workers [48] reported the room-temperature HDF of 1-fluorohexane 37 with $\mathrm{Ph}_{3} \mathrm{C}^{+}\left[\mathrm{B}\left(\mathrm{C}_{6} \mathrm{~F}_{5}\right)_{4}\right]^{-}$as the percatalyst (Scheme 9). However, it is less efficient than the silylium ion-based protocols and limited to monofluorinated hydrocarbons. And choosing Reed's halogenated carborane $\left[\mathrm{CHB}_{11} \mathrm{H}_{5} \mathrm{Br}_{6}\right]^{-}$as the supporting counteranion, the defluorinative alkylation competed strongly with the HDF reaction via the alkyl transfer from ${ }^{i} \mathrm{Bu}_{2} \mathrm{AlH}$ [49].

It is worthy of mention that Ehm and Lentz's group [50] developed an organocatalytic HDF with alkanes as the electrophiles (Scheme 10). This research demonstrated that

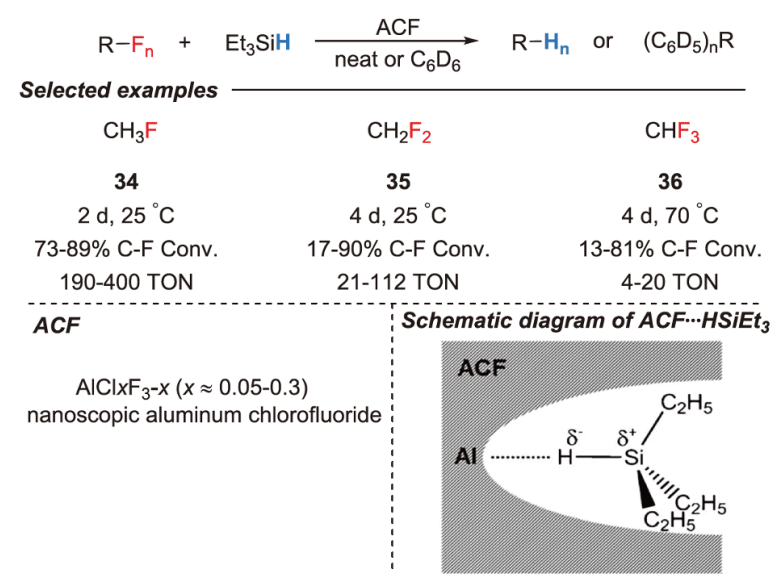

Scheme 8 ACF-catalyzed HDF of fluoromethanes (color online). 
donor solvents activate the aluminium hydride bond, lower the barrier for HDF significantly, and switch the product preference from $Z$ to $E$. Polyfluorinated compounds such as 41, 42, 43, 44 underwent HDF to deliver corresponding products when ${ }^{i} \mathrm{Bu}_{2} \mathrm{AlH}$ was employed as the $\mathrm{H}$ source.

In a practical contribution, Crimmin and co-workers [51] recently reported the selective HDF of hexafluoropropene $\mathbf{4 5}$ to industrially relevant hydrofluoroolefins (a new class of refrigerants) (Scheme 11). The use of $\mathrm{AlH}_{3} \cdot \mathrm{NMe}_{3}$ leads to the efficient and highly selective formation of $\mathbf{4 6}$ (HFO1234yf) under mild conditions. The mechanism experiment and the DFT calculations suggest that a concerted $\mathrm{S}_{\mathrm{N}} \mathrm{V}$ mechanism occurs with high substrate bias for $\mathrm{H} / \mathrm{F}$-exchange at the terminal position of $\mathbf{4 5}$.

\subsection{Germylium electrophiles}

In analogy to the HDF using silicon and aluminium electrophiles, it has been shown that germane can be employed as the hydrogen source or the per-catalyst. Different from Crimmin's work to synthesize the HFO-1234yf, Kemnitz and Braun's group [52] reported a consecutive transformation of it (46 or 47) with germane as the H source (Scheme 12). Treatment of $\mathbf{4 7}$ with $\mathrm{Et}_{3} \mathrm{GeH}$ in the presence of $\mathrm{ACF}$ (vide supra) under photolytic condition, the HDF products 49 and 50 were generated. Note that the irradiation is for the hydrogermylation step of the double bond $(\mathbf{4 8 , 5 0})$, and the presence of ACF in the reaction mixture is for HDF.

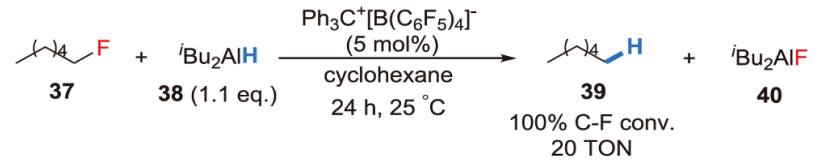

Scheme 9 Catalytic HDF with aluminium ions (color online).

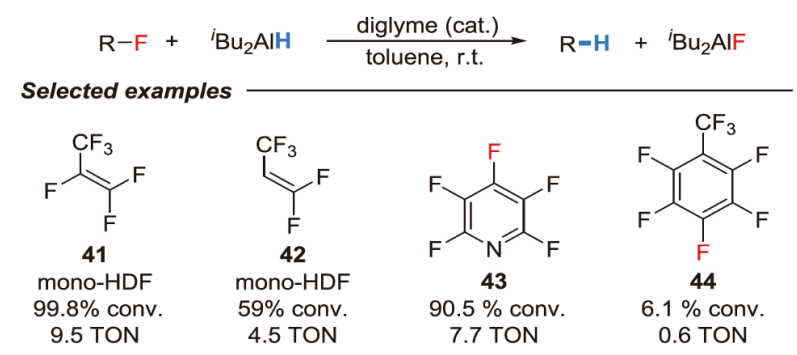

Scheme 10 Organocatalytic HDF with alanes (color online).

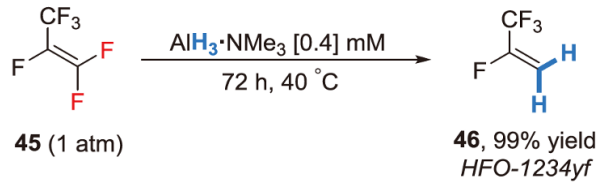

Scheme 11 Selective HDF of hexafluoropropene (color online).
Subsequently, Weinert's group [53] reported a roomtemperature HDF of acid fluorides $(\mathbf{5 1}, \mathbf{5 2})$ and alkyl fluorides $(\mathbf{5 3}, \mathbf{5 4})$. It postulated germylium cation $\mathrm{Ph}_{3} \mathrm{Ge}^{+}$as the active catalyst, generated in situ from germane $\mathrm{Ph}_{3} \mathrm{GeH}$ and a trityl salt $\mathrm{Ph}_{3}^{+}\left[\mathrm{B}\left(\mathrm{C}_{6} \mathrm{~F}_{5}\right)_{4}\right]^{-}$(Scheme 13). Interestingly, there is no decarbonylation or over-reduction of the acid fluorides.

\subsection{Photoinduced HDF}

In recent years, photocatalytic HDF has emerged as a promising, conceptually novel strategy to activate $\mathrm{C}-\mathrm{F}$ bonds selectively. Unlike strong Lewis acid for $\mathrm{C}-\mathrm{F}$ activation via fluoride abstraction, the photoredox protocol undergoes a single electron transfer (SET) process. Therefore, it's compatible with more functional groups and atoms which are incompatible with strong Lewis acids.

In 2016, Zhang and co-workers [54] reported a polyfluoroarene-arene (" $\pi$-hole- $\pi$ " [55]) interaction promoted photocatalytic HDF (Scheme 14). Using pyrene-based photocatalyst (PC1), $N, N$-diisopropylethylamine (DIPEA) as the base, common polyfluoroaromatics including hexafluorobenzene (HFB, 55), pentafluorobenzene (PFB, 56), pentafluoropyridine (57), and octafluoronaphthalene (58) could be successfully converted in good yields. And this catalytic system is compatible with an array of functional groups such as ester (59), $\mathrm{CF}_{3}(\mathbf{6 0})$, ether (61), and aryl (62). Notably, the 1,2,4,5-TFB (56) is also possible to be synthesized directly from HFB via one-pot di-HDF when a twofold base was used (see ref. for details). In addition to this, 24,250 TON was obtained when $0.002 \mathrm{~mol} \%$ of PC1 was used for the HDF of HFB. The authors proposed a weak " $\pi$ hole- $\pi$ " interaction promoted inner-sphere electron transfer (ET) reaction pathway based on the mechanism studies. Interestingly, the size and the shape of the photocatalyst for this conversion can be fine-tuned to enhance the overall catalytic

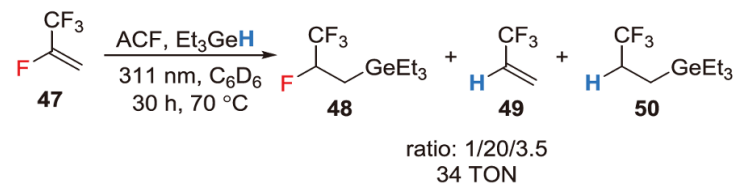

Scheme 12 Germane as the H source of ACF-catalyzed HDF (color online).

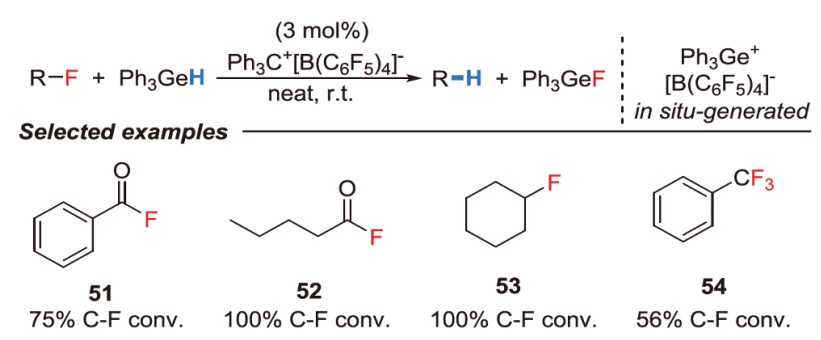

Scheme 13 Germane promoted catalytic HDF (color online). 


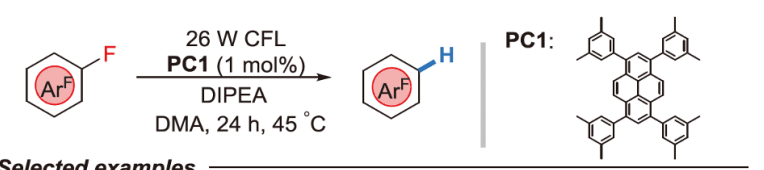

Selected examp or $48,5 \%$ yield
up 24,250 TON up 24,250 TON<smiles>CCOC(=O)c1c(F)ccc(F)c1F</smiles><smiles>Fc1cc(F)c(F)cc1F</smiles>

$56,80 \%$

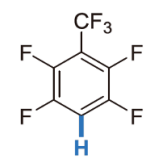

$60,75 \%$

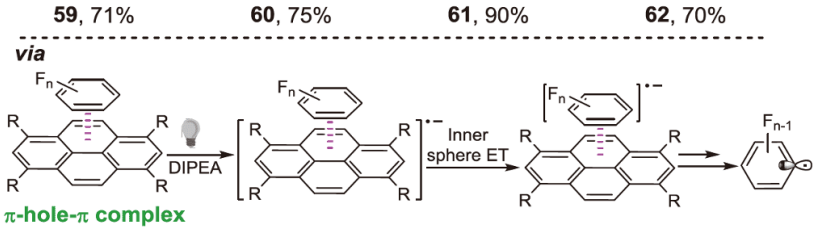

Scheme $14 \pi$-Hole- $\pi$ interaction promoted photocatalytic HDF (color online).

activity.

Recently, Jui's group [56] developed an elegant photoinduced selective HDF of unactivated trifluoro methylarenes (Scheme 15). The reaction conditions involved using cesium formate (3 equiv.) along with PC2 (2 mol\%) as the photocatalyst delivering the mono-HDF product 63 in $75 \%$ yield, without detectable over-reduction products. Functional groups such as cyano, amino (64), ether, hydroxyl (65), triflate (67), and alkyl (68) are compatible. Trifluoromethyl substituted pyridines 69,70 were also successfully transformed in this protocol, and the yields of pyridine-based substrates were bolstered by substituting electron-donating groups (EDG) at the ortho-position. The authors proposed that this strategy is achieved through an endergonic electron transfer event that provides access to arene radical anions that lie outside the catalyst reduction potential.

Subsequently, Gouverneur and co-workers' research [57] on selective mono-HDF of trifluoromethylarenes with electron-withdrawing groups (EWG) further significantly expanded the application scope (Scheme 16). Functionalities on the aromatic ring, including cyano $(\mathbf{7 1}, \mathbf{7 2})$, fluorine (72), ester (73), unprotected sulfonamide (74), were tolerated. The authors also examined complex molecules of biological relevance. For example, the doubly trifluoromethylated cannabinoid receptor agonist $B A Y$ 59-3074 reacted exclusively at the arene (75). Bicalutamide, a drug used to treat prostate cancer, underwent HDF affording $\mathbf{7 6}$ in 50\% yield and high $\mathrm{CF}_{2} \mathrm{H} / \mathrm{CH}_{2} \mathrm{~F}$ selectivity. Remarkable, an analogue of $\mathrm{En}$ obosarm, which has three trifluoromethylaryl groups, further demonstrated the chemoselectivity of the protocol. The HDF occurred at a single site with excellent $\mathrm{CF}_{2} \mathrm{H} / \mathrm{CH}_{2} \mathrm{~F}$ se-

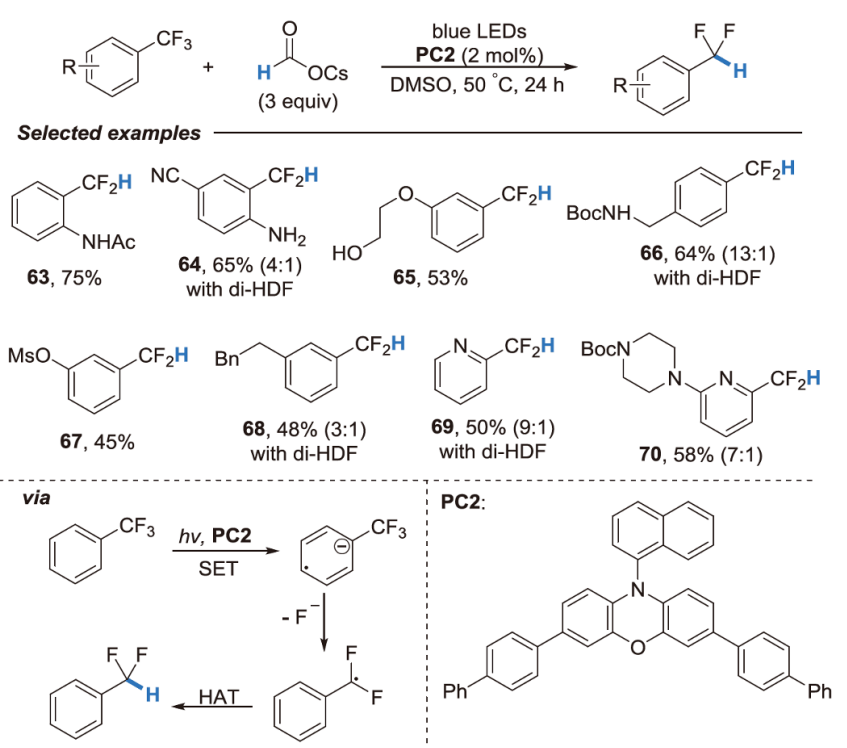

Scheme 15 Selective photocatalytic HDF of trifluoromethylarenes (color online).

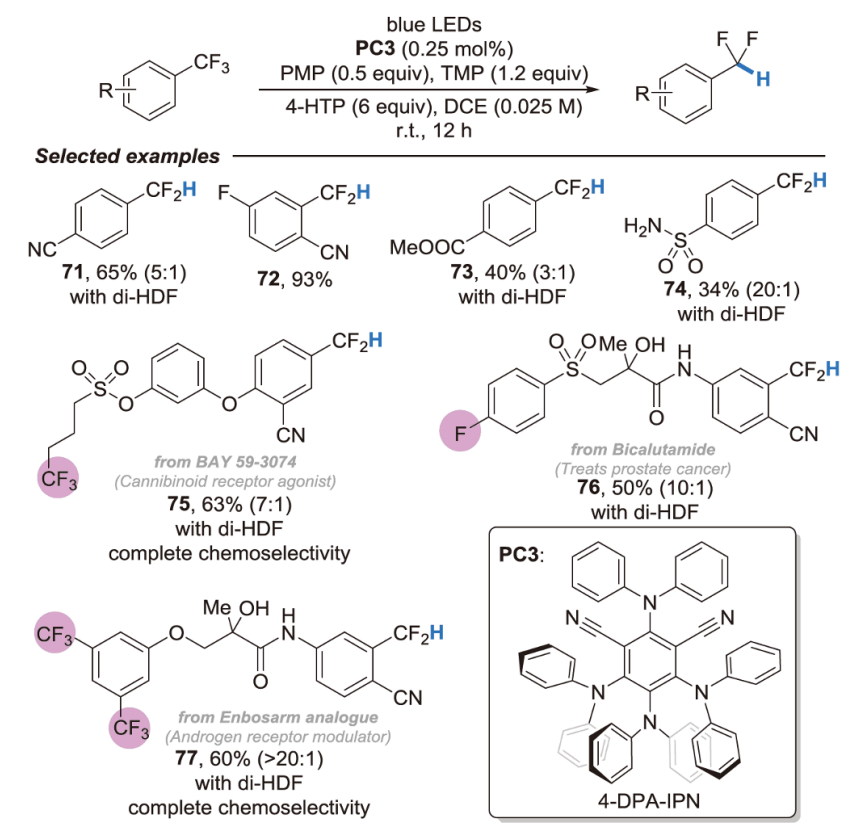

Scheme 16 Organophotoredox HDF of trifluoromethylarenes with EWG (color online).

lectivity, leaving the 3,5-bis-trifluoromethylarene motif untouched (77). The mechanistic studies suggest that the photocatalyst is reduced by the hydrogen atom donors and returned to its native oxidation state by the trifluoromethylarene that acts as an oxidant.

\subsection{Other electrophiles}

In addition to the nucleophilic reagents mentioned above, there are some other interesting approaches to HDF. Simi- 
larly, for the selective HDF of bis(trifluoromethyl)arenes, Prakash's group [58] used $\mathrm{Mg}$ powder to activate the C-F bond. It is worth mentioning that no mixture of bis- $\mathrm{CF}_{2} \mathrm{H}$, $\mathrm{CF}_{2} \mathrm{H} / \mathrm{CH}_{2} \mathrm{~F}$, or $\mathrm{CF}_{2} \mathrm{H} / \mathrm{CH}_{3}$-containing compounds was detected in the reactions (78-80) (Scheme 17). The reason explains that those $\mathrm{CF}_{2} \mathrm{H}$-containing aromatics are less likely to accept an electron from $\mathrm{Mg}^{0}$ due to the lower electronwithdrawing ability than $\mathrm{CF}_{3}$-containing aromatics. Therefore, bis(trifluoromethyl)arenes are prone to accept an electron for the $\mathrm{HDF}$ than the corresponding $\mathrm{CF}_{2} \mathrm{H} / \mathrm{CF}_{3}$ containing analogues. Once a $\mathrm{CF}_{2} \mathrm{H} / \mathrm{CF}_{3}$-containing compound accepts an electron, the $\mathrm{CF}_{2} \mathrm{H}$ group preferentially undergoes $\mathrm{HDF}$ due to lower $\mathrm{BDG}$ than the $\mathrm{CF}_{3}$ group. The scope also showed good compatibility with hydroxyl (81), aldehyde (82), and acetyl (83). However, the complete HDF products were delivered when extended $\pi$-system or nitrilecontaining substrates were used $(\mathbf{8 5}, \mathbf{8 6})$, reflecting some shortcomings of this protocol. The modifications of the drugs were successfully proceeded and provided mono-HDF products in moderate yields $(\mathbf{8 6}, \mathbf{8 7})$.

Gallium hydrides such as ${ }^{i} \mathrm{Bu}_{2} \mathrm{GaH}, \mathrm{LiGaH}_{4}$, and $\mathrm{Me}_{3} \mathrm{~N} \cdot \mathrm{GaH}_{3}$ can also be used as the electrophiles of HDF reactions (Scheme 18), which are illustrated by Ehm and Lentz et al. [59]. Employing diglyme as the donor (vide supra), quantitative conversion to the HDF products could be observed for hexafluoropropene (88) and 1,1,3,3,3-pentafluoropropene (89), 94\% conversion of pentafluoropyridine (90) and $49 \%$ of octafluorotoluene (91). Compared with their previous work (Scheme 10), the gallium hydrides are a better $\mathrm{H}$ source than aluminium hydrides.

An accidental example from García's group [60] showed that phosphine $\mathrm{Et}_{3} \mathrm{P}$ could act as a fluoride acceptor to pro-

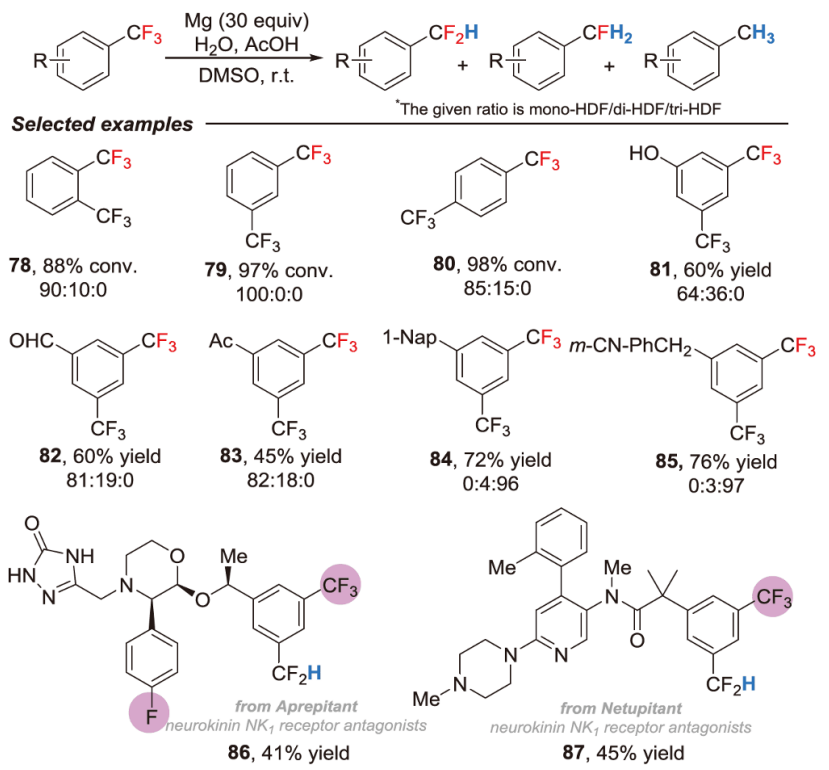

Scheme 17 Selective HDF of bis(trifluoromethyl)arenes promoted by Mg powder (color online). mote the HDF reaction (Scheme 19). Note that the mechanism initially proposed was amended by their subsequent work [61]. In this conversion, the source of the hydrogen was the water in the reaction rather than $\mathrm{Et}_{3} \mathrm{P}$. Under the given system, polyfluoroarenes underwent single or $d i$-HDF to quantitatively provide the relatively stable fluorinated arenes (92-95). EWGs such as trifluoromethyl, acetyl, and cyano were tolerated well (96-98). To achieve this transformation, $\mathrm{Et}_{3} \mathrm{P}$ first nucleophilic attacks the polyfluoroaromatic ring with F-migration forming 99 and yielding intermediate $\mathbf{1 0 0 .}$ Then it reacts with water and produces ionic pair $\mathbf{1 0 2}$ via the transition state 101. The highest barrier corresponds to the first step; thus, it is considered the rate-determining step of the reaction.

In analogy to previous protocols which utilized ${ }^{i} \mathrm{Bu}_{2} \mathrm{AlH}$ or $\mathrm{LiGaH}_{4}$ (vide supra), Weaver and co-workers [62] reported the HDF of perfluoroarenes with $\mathrm{NaBH}_{4}$ (Scheme 20). The advantage of this reaction is that it is compatible with both EDG and EWG substituted substrates (103-114). Specifically, the use of $\mathrm{NaBH}_{4}$ rather than more potent reductant $\mathrm{LiAlH}_{4}$ allowed for the incorporation of ester (103) and amide (104). Both alkenyl (108) and alkynyl (109) sub-

$$
\mathrm{R}-\mathrm{F}+\underset{1.1 \text { equiv }}{\mathrm{LiGaH}_{4}} \stackrel{\text { diglyme (cat.) }}{\text { toluene, r.t. }} \mathrm{R}=\mathrm{H}
$$

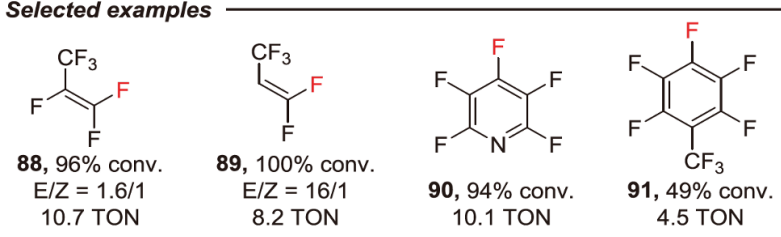

Scheme 18 Gallium hydrides for HDF (color online).

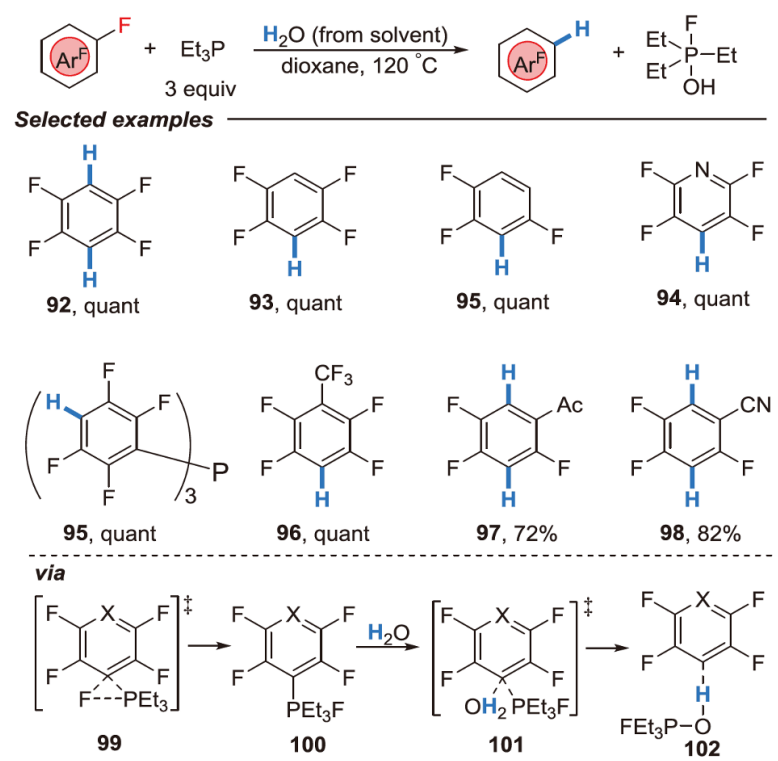

Scheme $19 \mathrm{HDF}$ with $\mathrm{Et}_{3} \mathrm{P}$ and $\mathrm{H}_{2} \mathrm{O}$ (color online). 
stituents were sufficiently activated to facilitate HDF. Unsurprisingly, substrates such as hexafluorobenzene or octafluoronaphthalene underwent di-HDF in the expected reaction site $(\mathbf{1 1 2}, \mathbf{1 1 3})$.

Very recently, Wang's group [63] published their research on the sequential $\mathrm{C}-\mathrm{F}$ functionalizations of trifluoroacetamides and acetates (Scheme 21). This breakthrough provides a straightforward manner for the selective formation of mono- and difluoroalkyl from readily accessible trifluoroacetic acid derivatives. They posited a discrete two-

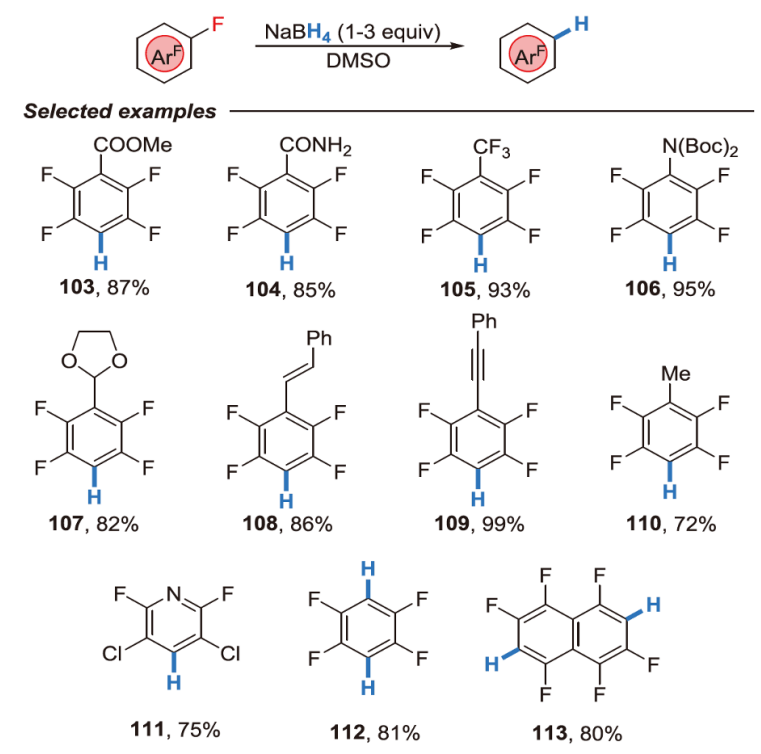

Scheme 20 HDF with $\mathrm{NaBH}_{4}$ (color online).

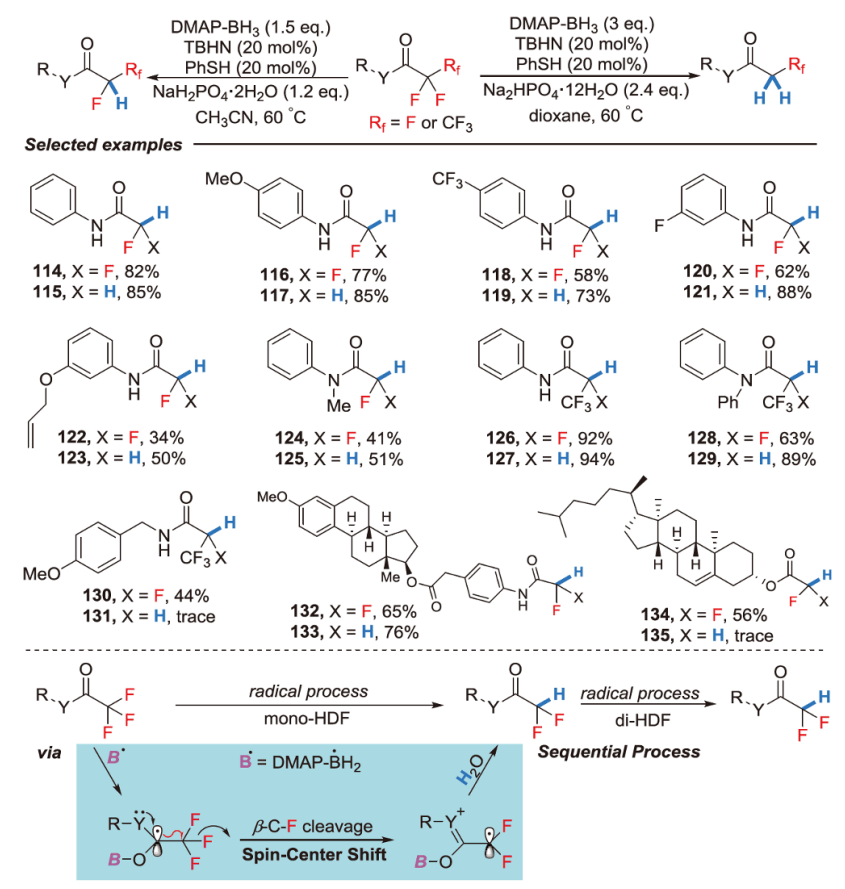

Scheme 21 Sequential HDF via spin-center shifts (color online). stage process with each stage involving a spin-center shift (SCS) [64-66] based on radical process, which involves 1,2radical delocalization and leaving group elimination for the $\mathrm{C}-\mathrm{F}$ bond cleavage. In this reaction system, 4-dimethylaminopyridine (DMAP)- $\mathrm{BH}_{2} \cdot$ as a radical initiato generated by hydrogen atom abstraction from $\mathrm{DMAP}-\mathrm{BH}_{3}$ using ditert-butyl hyponitrite (TBHN), to promote the defluorination. Thiophenol $(\mathrm{PhSH})$ was used as a polarity reversal catalyst. And in the presence of $\mathrm{NaH}_{2} \mathrm{PO}_{4} \cdot 2 \mathrm{H}_{2} \mathrm{O}$ (1.2 equiv.) as the base, various mono-HDF products were obtained. When increased the amounts of $\mathrm{DMAP}^{-\mathrm{BH}_{3}}$ ( 3 equiv.) and $\mathrm{Na}_{2} \mathrm{HPO}_{4} \cdot 12 \mathrm{H}_{2} \mathrm{O}$ (2.4 equiv.) in 1,4-dioxane, di-HDF products were selectively produced. Both methods showed broad substrates scope and good chemoselectivity (114123). Tertiary amides and pentafluoropropanamides reacted well under both methods (124-131). Only the $\mathrm{C}-\mathrm{F}$ bonds $\alpha$ to carbonyl group were selectively reduced, whereas the $\mathrm{CF}_{3}$ group was inert, suggesting that cleavage of $\mathrm{C}-\mathrm{F}$ bond might be assisted by the adjacent carbonyl moiety. Complex molecules containing $\beta$-estradiol or cholesterol framework were also successfully converted into corresponding products (132-134).

\section{Defluorinative $\mathrm{C}-\mathrm{C}$ bond formation}

\subsection{Aromatic $\mathrm{C}-\mathrm{F}$ bond cleavage}

The Grignard reagent is one of the most frequently used organometallic reagents to form $\mathrm{C}-\mathrm{C}$ bonds and occupies an important position in synthetic chemistry. Since Kumada et al. [67] reported the first Ni-catalyzed cross-coupling of fluorobenzene with Grignard reagent, investigations into this type of reaction gradually emerged. In a transition-metal-free manner, Cao's group [68,69] presented their studies of crosscoupling of polyfluoroarenes and gemdifluoroalkenes with Grignard reagents (Scheme 22). First, they utilized pyridine as the directed group. The $\mathrm{C}-\mathrm{F}$ bond ortho to the pyridine nitrogen could be selective cleaved (136-139). Three years later, they found that the steric effect played a very important role in stereoselective alkylation of gem-difluoroalkenes. The tertiary and secondary alkylation proceeded smoothly, affording alkyl-substituted fluoroalkenes in good to excellent yields with excellent $Z$ stereoselectivity (140-143).

Similarly, Li and co-workers [70,71] reported alkylation and arylation of fluorinated arenes with Grignard reagents (Scheme 23). By choosing the aldazine-N atom as the directing group, fluorinated aryl aldehydes were regioselectively synthesized (144-147). Subsequently, they described a simple method on direct nucleophilic substitution of polyfluoroarenes under mild conditions. Both alkyl and aryl Grignard reagents, as well as a variety of polyfluoroarenes, were amenable (148-151). It is worth noting that they ob- 

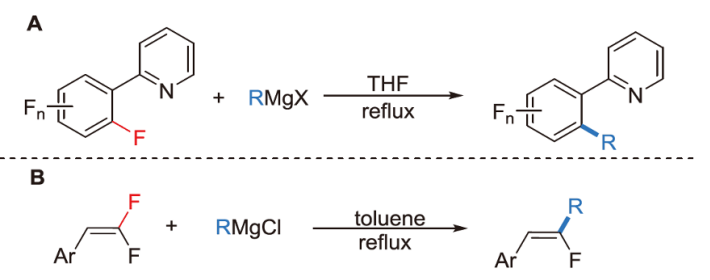<smiles>CC(C)(C)OC(=O)OCc1ccccn1</smiles>

$136,89 \%$

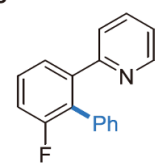

$137,83 \%$

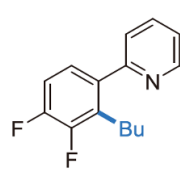

$138,83 \%$

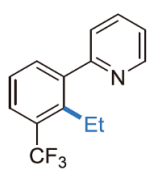

$139,89 \%$

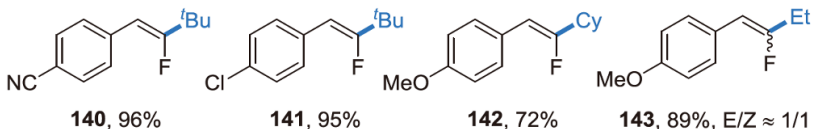

Scheme 22 Cao's approach on cross-coupling of polyfluoroarenes and gem-difluoroalkenes with Grignard reagents (color online). 作 B (Ar ${ }^{\mathrm{F}}+\mathrm{RMgCl} \underset{\text { r.t. } 24 \mathrm{~h}}{\mathrm{THF}}$ Arf $^{\mathrm{R}}$ Selected examples

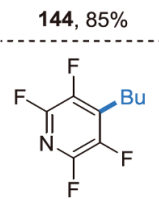

$148,99 \%$

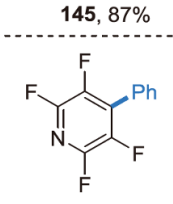

$149,85 \%$

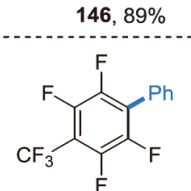

$150,85 \%$

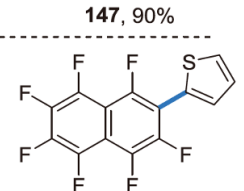

$151,60 \%$
Scheme 23 Li's approach on alkylation and arylation of fluorinated arenes with Grignard reagents (color online).

served this reaction was dramatically suppressed in the presence of the Ni catalyst, which was distinct from other Grignard reactions.

The demand for transition-metal-free polymerization methods stimulated the development of innovative $\mathrm{C}-\mathrm{F}$ functionalization protocols. Watson's group [72,73] and Iyoda's group [74] showed that Fluoride ion could activate the $\mathrm{C}-\mathrm{Si}$ bond of silylalkynes, thereby allowing for the polymerization with polyfluoroarenes (Scheme 24). The high molecular weight poly(phenylene ethynylene)s (PPEs) can be obtained by the simple $\mathrm{S}_{\mathrm{N}} \mathrm{Ar}$ reaction and the only side product is fluorotrimethylsilane (TMSF, gas).

The direct cross-coupling of polyfluoarenes and alkynes is possible. However, stoichiometric amount of strong base is usually required. For example, Cao's group [75,76], Zhang's group [77], and Kondo's group [78] reported those baseassisted alkynylations of the polyfluoroarenes (Scheme 25). The key to this type of transformation is the generation of the acetylene anion. Thus, the strong base is indispensable under transition-metal-free conditions.

For intramolecular reactions, Siegel and co-workers $[79,80]$ gave an impressive contribution (Scheme 26). They showed that phenyl cation, which is generated from otherwise unreactive aryl fluorides, allows extension of the Friedel-Crafts reaction to intramolecular aryl couplings. The reaction is activated by an intermediate silyl cation. The methodology allows the high-yield formation of a range of tailored polycyclic aromatic hydrocarbons and graphene fragments. This strategy could also lead to formal $\mathrm{C}-\mathrm{H}$ activation and the new $\mathrm{C}\left(\mathrm{sp}^{2}\right)-\mathrm{C}\left(\mathrm{sp}^{3}\right)$ bonds formation.

The intramolecular HF elimination reaction (cove-region closure process) is also the key transformation in the rational synthesis of bucky-bowl, nanographenes, and nanoribbons. Amsharov's group [81-84] demonstrated this type of transformation by utilizing $\gamma-\mathrm{Al}_{2} \mathrm{O}_{3}$ (Scheme 27). Only when the hydrogen atoms in the precursor structure are spatially adjacent can fluorine promote the desired ring closure (156159). The high efficiency of the approach provided the desired products with high purity without any additional purification procedures.

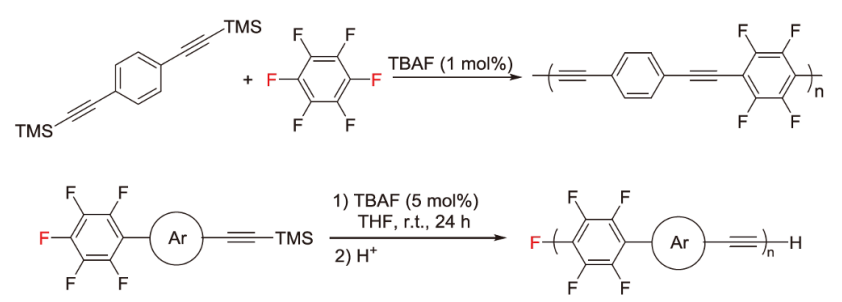

Scheme 24 Fluoride ion-catalyzed polymerization via F-Si bond formation (color online).
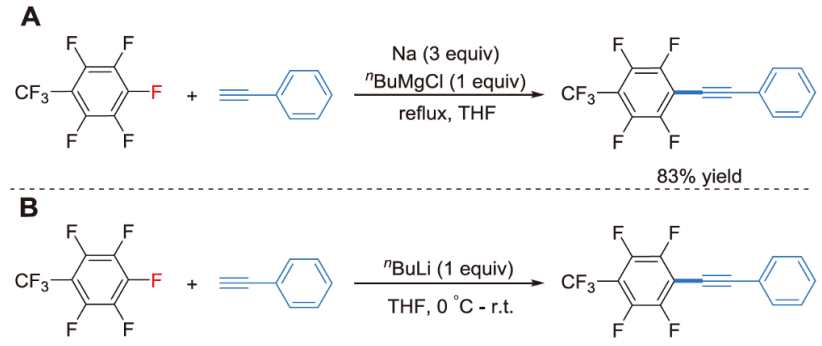

$83 \%$ yield
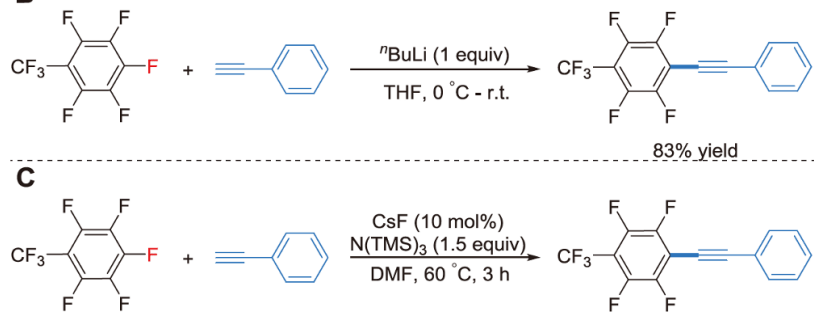

$83 \%$ yield
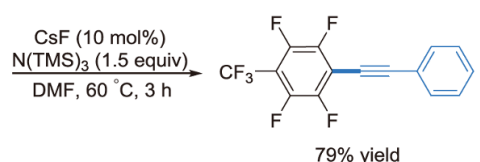

Scheme 25 Base-assisted Sonogashira-type coupling of polyfluoroarenes (color online). 


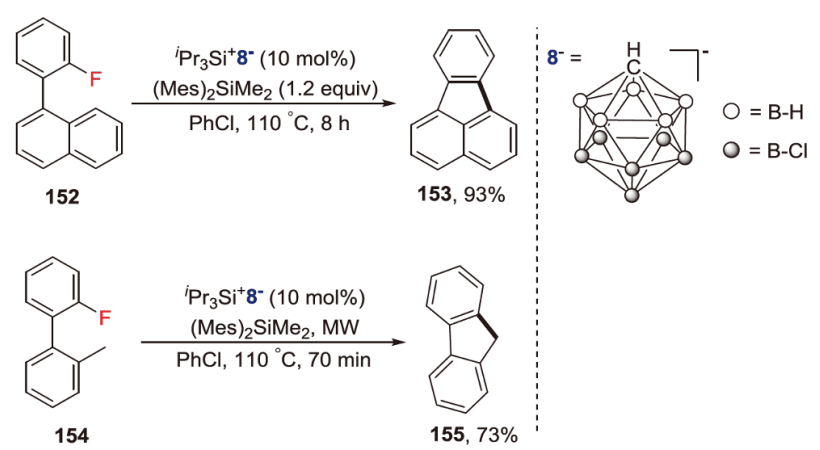

Scheme 26 Intramolecular Friedel-Crafts coupling of fluoroarenes (color online).

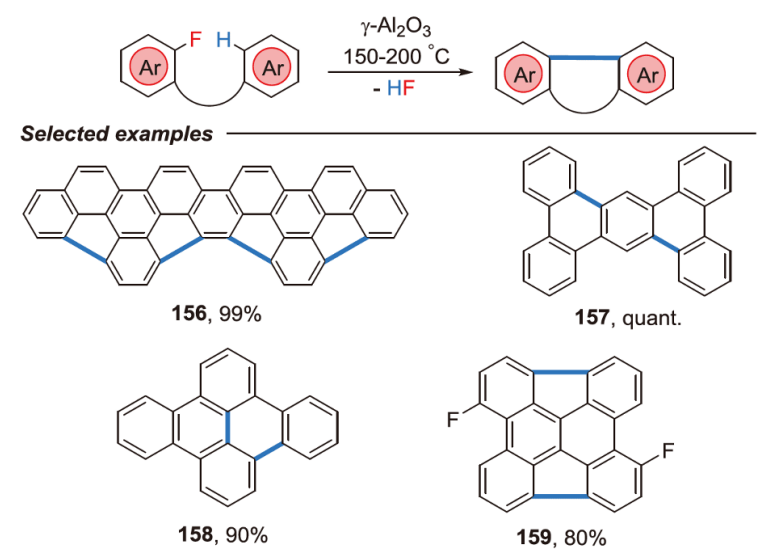

Scheme 27 Cove-region closure process with $\gamma-\mathrm{Al}_{2} \mathrm{O}_{3}$ (color online).

Interestingly, Ichikawa and co-workers [85] demonstrated that the construction of benzene-fused triphenylene frameworks is regioswichable (Scheme 28). On treatment with $\mathrm{AlCl}_{3}$ or $\gamma-\mathrm{Al}_{2} \mathrm{O}_{3}$, benzo[f]tetraphenes and benzo $[g]$ chrysenes could be selectively synthesized via aromatic $\mathrm{C}-\mathrm{F}$ bond cleavage of 2-(biphenyl-2-yl)-1-fluoronaphthalenes(cyclization precursors). The authors proposed that the high selectivity is due to the generation of fluorine-stabilized intermediary arenium ions.

Nelson's group [86] presented that the arenium ions can be stabilized by $\beta$-silicon and inserted into $\mathrm{sp}^{2}$ and $\mathrm{sp}^{3} \mathrm{C}-\mathrm{H}$ bond (Scheme 29). Phenyl halonium salt of undecachlorinated monocarba-closo-dodecaborate anion $(\mathbf{1 6 0})$ as pre-catalyst, $\beta$-silylated aryl fluorides as phenyl cation precursors, the weaker $\mathrm{C}-\mathrm{X}$ bonds that have less steric encumbrance than the $\mathrm{C}-\mathrm{F}$ bond did not undergo ionization (161). The alkanes could also be phenylated in moderated yield (164). Remarkably, the methane gas functionalization was successful (165) under the conditions. The key to this success is the $\beta$-silicon group stabilization lowers the barrier for fluoride abstraction and temper the $\sigma$-electrophilicity of the resulting phenyl cations, thus generated the key reactive intermediate ( $\beta$-silicon stabilized arenium).

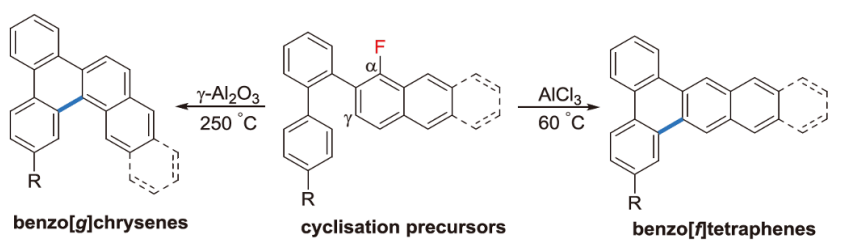

Scheme 28 Regioswichable synthesis of polycyclic aromatic hydrocarbons (color online).

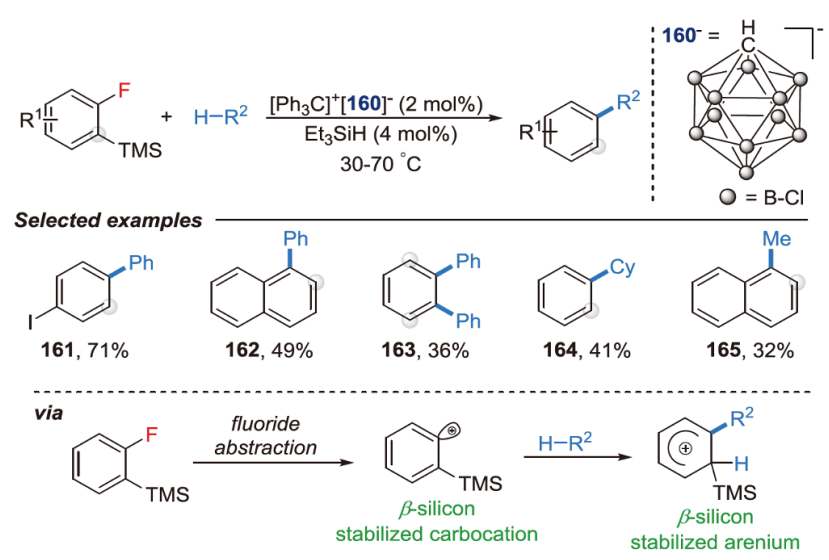

Scheme 29 Arylation of hydrocarbons with $\beta$-silicon stabilized arenium ions (color online).

\subsection{Benzylic and allylic C-F bonds cleavage}

The Friedel-Crafts type reaction, classical $\mathrm{C}-\mathrm{C}$ bond formation reaction, is an important tool for building $\mathrm{C}-\mathrm{C}$ bonds. Ozerov's group [30-32] and Müller's group [35] already noticed this type of reaction in their silylium ion-catalyzed HDF contributions (vide supra). When (3,3,3-trifluoropropyl)benzene 7 was treated with the silylium ion catalyst in benzene as the solvent, and no HDF product was observed (Scheme 3). Instead, the defluorinative FriedelCrafts type $\mathrm{C}-\mathrm{C}$ bond formation product 9 was obtained as the major product. A well-conceived approach on benzylation and alkylation of aryl and alkyl $\mathrm{CF}_{3}$ groups was then reported by Stephan et al. [87] (Scheme 30). This metal-free procedure involves sequential benzylation or alkylation and HDF reactions. The difluorocarbocation is generated by the Lewis acid catalyst $\left[\left(\mathrm{C}_{6} \mathrm{~F}_{5}\right)_{3} \mathrm{PF}\right]\left[\mathrm{B}\left(\mathrm{C}_{6} \mathrm{~F}_{5}\right)_{4}\right]$ and then undergoes Friedel-Crafts type electrophilic aromatic substitution. The resulting intermediate reacts with silane through HDF to deliver the target molecules and release $\mathrm{H}_{2}$. With this protocol, various arenes or trifluoromethyl aryl species such as $\mathrm{C}_{6} \mathrm{D}_{6}$, 1,2,3,4,5-pentamethylbenzene, halobenzene, and naphthalene, were converted into diarenes in moderate to high yields $(\mathbf{1 6 6}-\mathbf{1 7 1})$. The alkyl $\mathrm{CF}_{3}$ substrates have analogous reactivity and delivered the corresponding products 172-174 in good yields. The coordination of the pyrazole derivative to the phosphonium Lewis acid catalyst makes the 


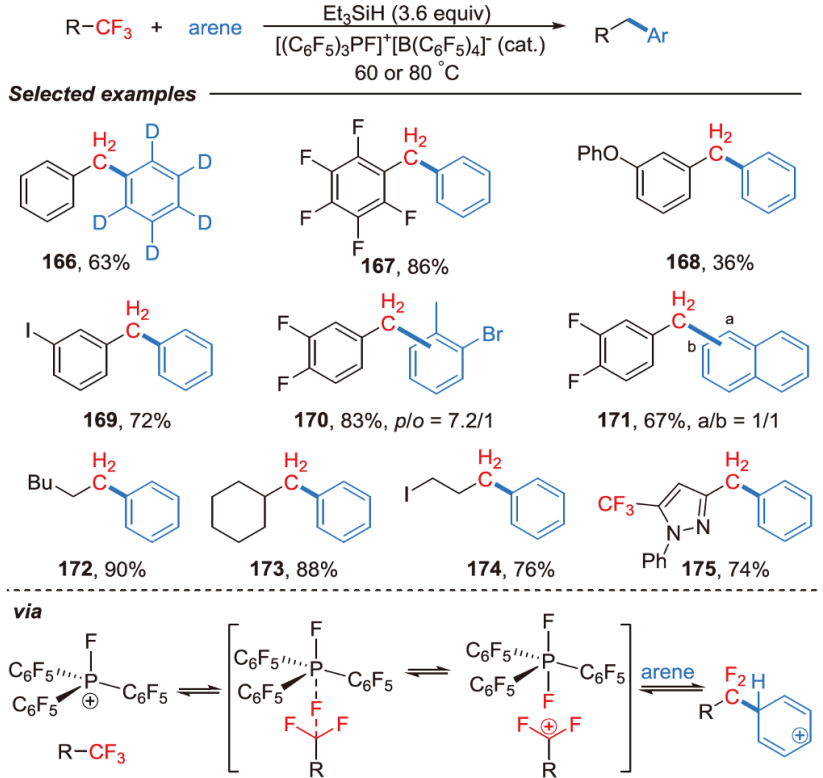

Scheme 30 Catalytic benzylation and alkylation of trifluoromethyl groups (color online).

$\mathrm{N}$ atom incompatible under this catalytic system. However, the addition of 1 equivalent of $\mathrm{B}\left(\mathrm{C}_{6} \mathrm{~F}_{5}\right)_{3}$ to the reaction mixture, resulting in the clean formation of the benzylated product $\mathbf{1 7 5}$ in $74 \%$ yield. This observation suggests that coordination of the pyrazole to the more accessible borane allows $\mathrm{C}-\mathrm{F}$ activation and electrophilic substitution.

Similarly, for converting the stable $\mathrm{CF}_{3}$ group, Yoshida's group [88] developed a boron tribromide-mediated FriedelCrafts-type acylation of arenes with benzotrifluoride (Scheme 31). Through the three-times defluorobromination of benzotrifluoride to generate the tribromide, then reacted with methanol and arene, various diaryl ketones (176-178) and aromatic esters (179-182) were synthesized. This protocol provided an alternative method for the formation of diaryl ketone. The success of this transformation is that consistent with the relative Lewis acid strengths of the boron halides, the order of catalyst activity is $\mathrm{BI}_{3}>\mathrm{BBr}_{3}>\mathrm{BCl}_{3}>$ $\mathrm{BF}_{3}$. Thus, complete bromodefluorination of $\mathrm{C}\left(\mathrm{sp}^{3}\right)-\mathrm{F}$ bonds can be achieved using stoichiometric amounts of $\mathrm{BBr}_{3}$ $[89,90]$.

The strategy for the formation of TMSF to break the C-F bond can be extended to trifluoromethylarenes. In 2016, Hosoya's group [91] reported a single C-F bond cleavage of trifluoromethylarenes with an ortho-silyl group (Scheme 32). The activation of the hydrosilyl group with a trityl cation in the presence of nucleophiles allowed for selective $\mathrm{C}-\mathrm{F}$ bond functionalization (183-189). The authors proposed that the reaction was triggered by hydride abstraction or underwent a concerted pathway involving hydride abstraction and $\mathrm{C}-\mathrm{F}$ bond cleavage.

Subsequently, Bandar and co-workers [92] developed a

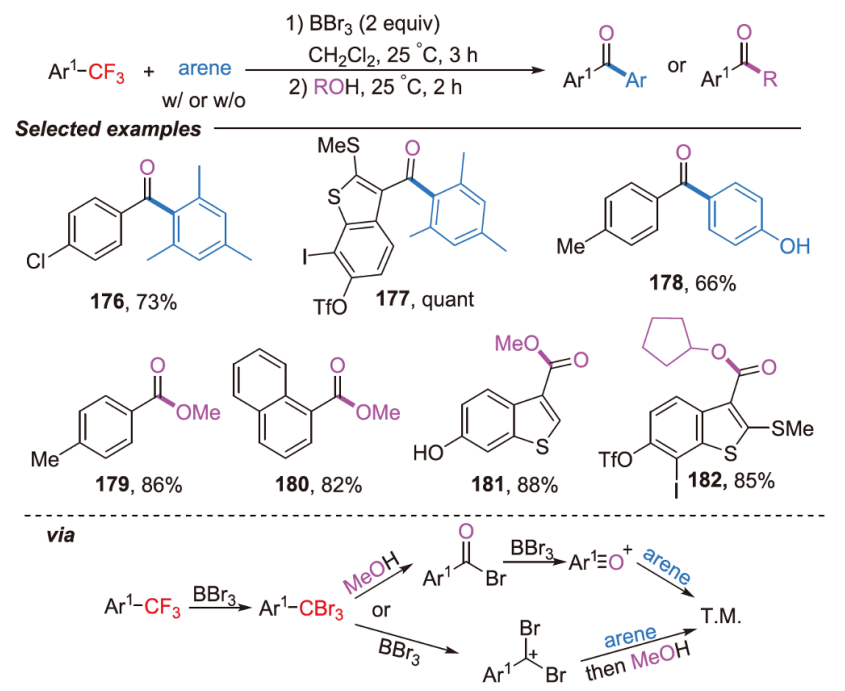

Scheme $31 \mathrm{BBr}_{3}$-mediated $\mathrm{C}-\mathrm{F}$ cleavage of aryl $\mathrm{CF}_{3}$ groups (color online).

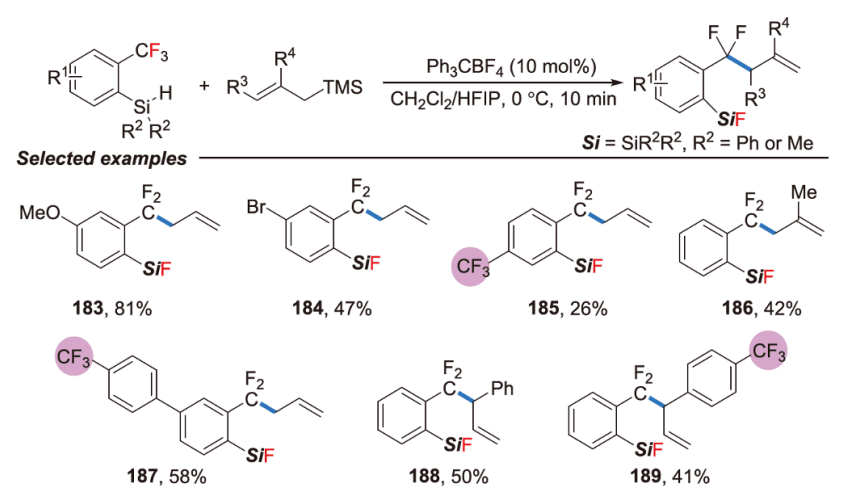

Scheme 32 ortho-Silyl group-assisted $\mathrm{C}-\mathrm{F}$ bond cleavage of trifluoromethylarenes (color online).

fluoride-initiated coupling reaction between trifluoromethylarenes and allylsilanes to access allylated $\alpha, \alpha$ difluorobenzylic compounds (Scheme 33). This strategy employed fluoride ions as promoters to break the $\mathrm{C}-\mathrm{Si}$ bond and then facilitated the cleavage of the $\mathrm{C}-\mathrm{F}$ bond. The 1,3bis(trifluoromethyl)arenes underwent allylation of one trifluoromethyl group in high yield (190). Heterocyclic substrates were similarly effective $(\mathbf{1 9 1}, \mathbf{1 9 2})$. 2-Substituted allyltrimethylsilanes yielded disubstituted alkene product 193 in good yield. Unlike the Hosoya's work, the initial mechanistic studies suggested a base-induced single electron transfer pathway is involved in this transformation. Thus, the allyl radical species that are generated only in the presence of a suitable trifluoromethylarene substrate (194-196). In addition to using fluoride ion, this transformation can actually be achieved with a hemilabile ligand coordinated P(III) complex developed by Stephan's group [93] (not shown).

The radical-based process for C-F bond cleavage was reported by Jui's group $[56,94]$ (Scheme 34). They developed 
photocatalytic selective functionalization of the $\mathrm{C}-\mathrm{F}$ bonds in trifluoromethylaromatic systems. First, they choose $\mathrm{N}$ phenylphenothiazine (PTH), introduced by Read de Alaniz and Hawker's group [95], as the organic photoredox catalyst, which would generate the highly reducing excited state $\mathrm{PTH}^{*}\left(E_{1 / 2} *=-2.10 \mathrm{~V} v s\right.$. SCE $)$. The catalytic system can activate the 1,3-bistrifluoromethylbenzene $\left(E_{1 / 2}^{0}=-2.07 \mathrm{~V}\right.$ $v s$. SCE) via single electron transfer (SET) and deliver the radicals, which are captured by the alkenes and provide the target molecule (T.M.) via HAT. Although the protocol is limited to substrates with EWG, it is a significant breakthrough in the field of transition-metal-free $\mathrm{C}-\mathrm{F}$ bond cleavage. Subsequently, the authors found that the PC2, developed by Miyake's group [96] for organic atom-transfer radical polymerization, was a more effective catalyst for this type of reaction. It enabled a wider range of substrates to be applied.

Activation of the allylic $\mathrm{C}-\mathrm{F}$ bond could be accomplished through treatment with a Lewis acid (Scheme 35), which was demonstrated by Ichikawa's group [97]. In the presence of

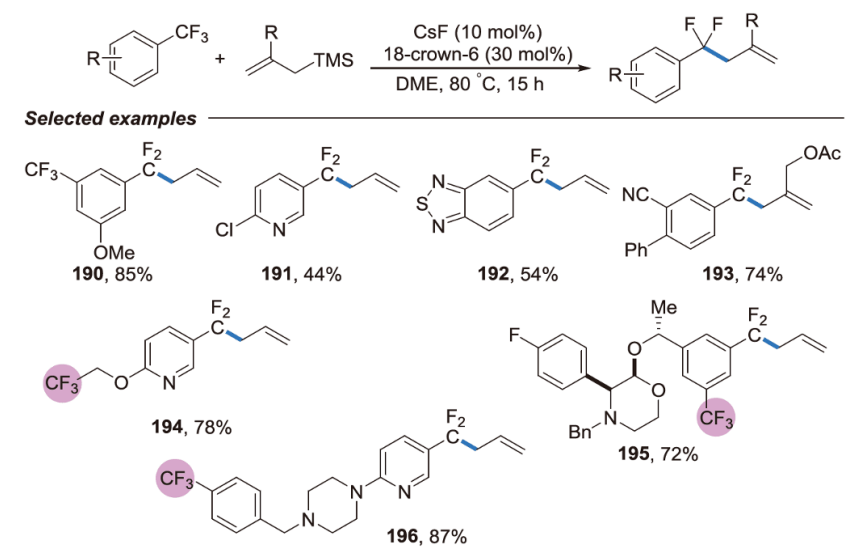

Scheme 33 Fluoride ion-catalyzed defluoroallylation of trifluoromethylarenes (color online).

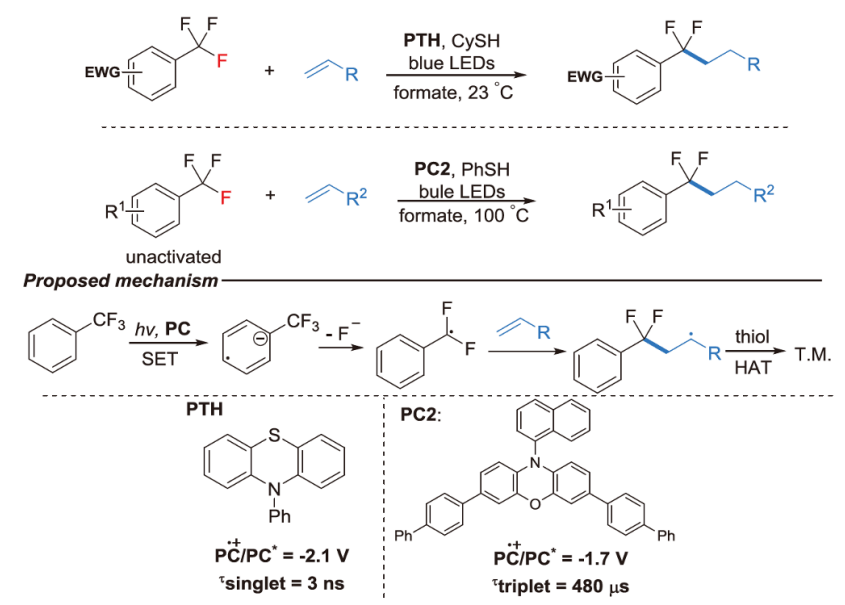

Scheme 34 Light-driven C-F bond cleavage to form $\mathrm{C}-\mathrm{C}$ bond (color online). stoichiometric $\mathrm{EtAlCl}_{2}$, the (trifluoromethyl)alkenes readily eliminated $\mathrm{F}^{-}$and underwent cationic substitution with arenes to produce 3,3-difluoroallylated arenes (197-202) in good yields. The silyl ether $\mathbf{2 0 2}$ was isolated after the quenching procedure. The regioselectivity showed in this reaction suggests a Friedel-Crafts-type mechanism for the $\mathrm{C}-\mathrm{C}$ bond-forming step. The fluorine stabilized allylic $\mathrm{CF}_{2}$ cations might be the key intermediate in this transformation.

An example on enantioselective $\mathrm{C}-\mathrm{C}$ bond formation via C-F bond cleavage was achieved by Shibata et al. [98-101] by using (DHQD) $)_{2}$ PHAL, a bis(cinchona alkaloid), as the catalyst (Scheme 36). Their elegant and detailed research demonstrated that with the assistance of (DHQD) $)_{2}$ PHAL, the allylic monofluorides could be kinetically split and reacted enantioselectively with the TMS reagents, such as RuppertPrakash reagent $\left(\mathrm{TMSCF}_{3}\right)$, alkynylsilanes, and (tetrazolyl) methylsilanes.

\subsection{Vinylic and aliphatic $\mathrm{C}-\mathrm{F}$ bond cleavage}

Ichikawa's group [102-106] developed a series of FriedelCrafts-type cyclization of 1,1-difluoroallenes to synthesize helicenes and polycyclic aromatic hydrocarbons (PAHs)

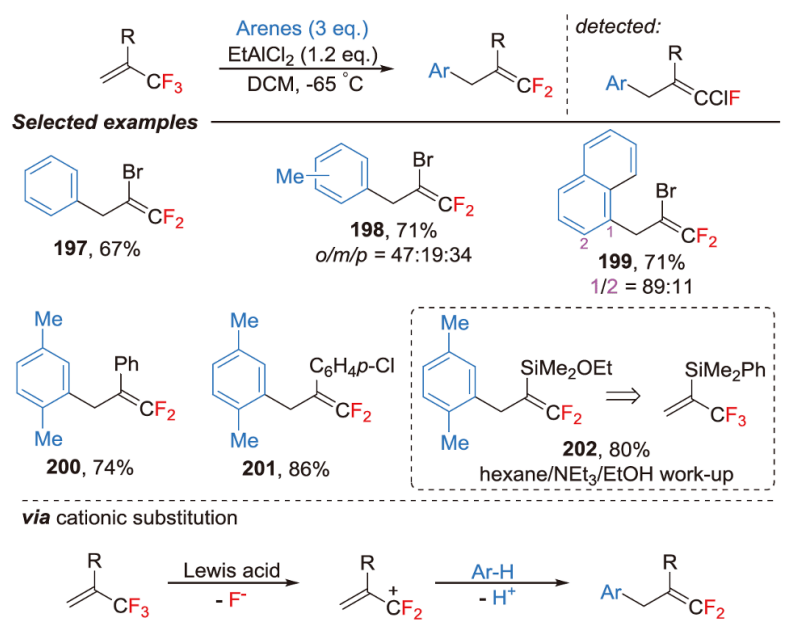

Scheme 35 Lewis acid promoted allylic C-F bond activation (color online).

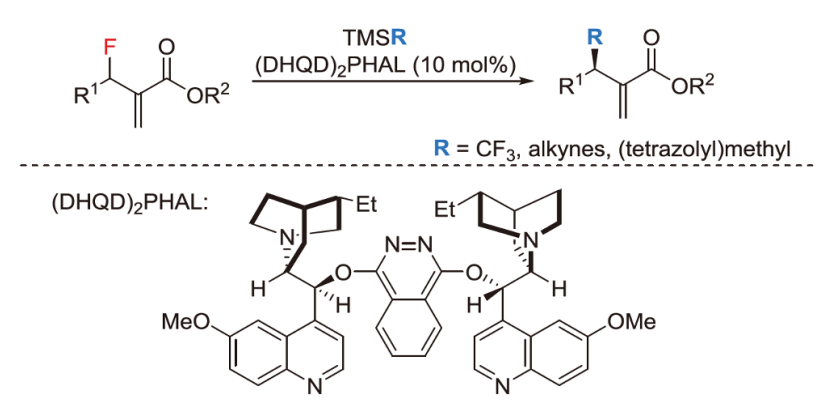

Scheme 36 Enantioselective $\mathrm{C}-\mathrm{C}$ bond formation via (DHQD) ${ }_{2} \mathrm{PHAL}$ (color online). 
(Scheme 37). Utilizing highly acidic reagents such as magic acid $\left(\mathrm{FSO}_{3} \mathrm{H} \cdot \mathrm{SbF}_{5}\right)$ and $\mathrm{TfOH}$, or Lewis acid $\mathrm{TiF}_{4}$, the 1,1difluoroalkenes were successfully converted into desired products via the generation of the $\mathrm{CF}_{2}$ cations. After the dehydrogenation step, PTHs were then obtained. The authors' research suggested that the $\mathrm{CF}_{2}$ cations can be accessed easier by introducing an electron-rich group (alkene) to 1,1-difluoroalkenes (Scheme 37, A and B). Remarkably, the catalytic process was also developed. With $\mathrm{InBr}_{3}$ as the catalyst, the 1,1-difluoroallene (Scheme 37, D) was transformed into the allylic $\mathrm{CF}_{2}$ cation, which undergoes FriedelCrafts-type cyclization and 1,2-migration, ring expansion to provide the product.

Shibata's group [107,108] developed another type of intramolecular ring-closure reaction (Scheme 38). They described the $\mathrm{B}\left(\mathrm{C}_{6} \mathrm{~F}_{5}\right)_{3}$-catalyzed intramolecular cascade Friedel-Crafts defluorinative cyclization of inert $\mathrm{C}\left(\mathrm{sp}^{3}\right)-\mathrm{F}$ bonds. It is worth noting that in the absence of a hydrogenbonding donor solvent HFIP, the aliphatic gem-difluorides preferentially engage in a defluorination/elimination process that provides monofluorinated alkenes (not shown).

Aluminum as a Lewis acid could also facilitate the coupling of the silylalkynes with the C-F bonds [49]. Young's group [109] demonstrated that ACF (vide supra) performed well in of catalytic incorporation of alkynes into aliphatic $\mathrm{C}-\mathrm{F}$ positions (Scheme 39). This method applies to primary, secondary, and tertiary fluorides. The fluorophilicity of the

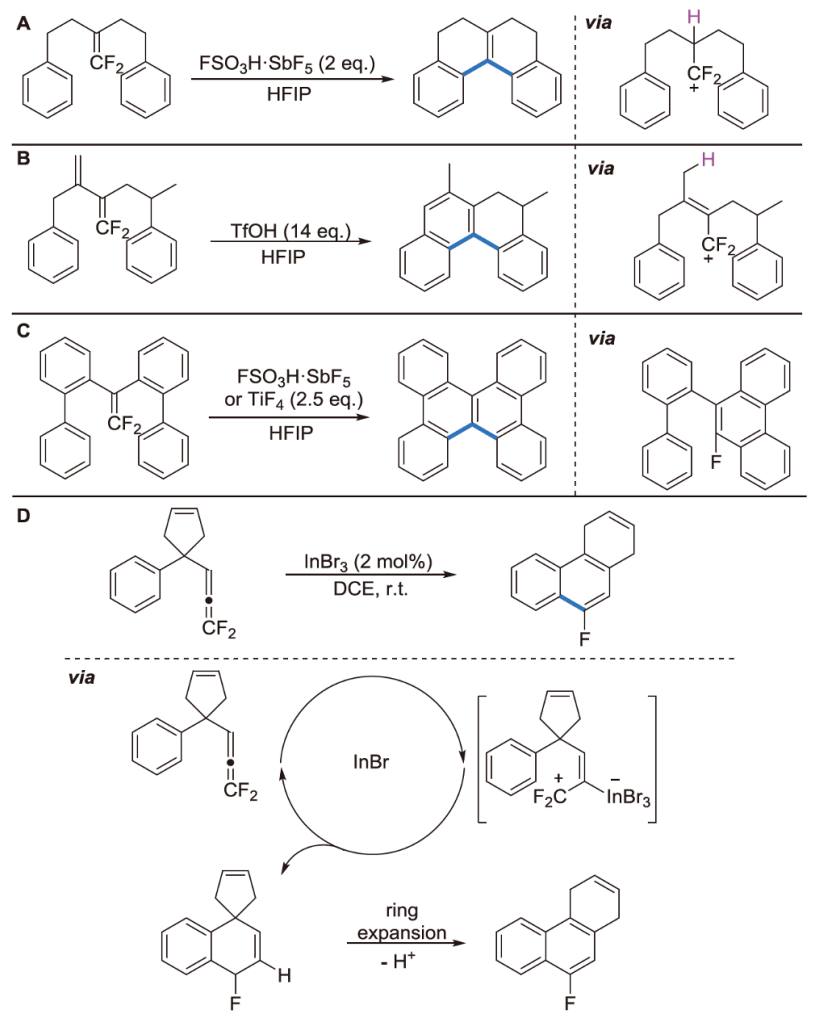

Scheme 37 Domino synthesis of PAHs from 1,1-difluoroallenes (color online).
$\mathrm{Al}$ catalyst gives rise to fluorine selectivity over other halogens.

Interestingly, Tobisu's group [110] reported a phosphinecatalyzed intermolecular acylfluorination of alkynes using acyl fluorides as the fluorinating reagents (Scheme 40). Halogen groups (207) were compatible in the reactions. The ratio of isomers was determined under thermodynamic. However, when substrates with a 2-pyridine group were used, the products were obtained with high $Z$ selectivity (208-210). Different electron-withdrawing groups (EWG) such as a tert-butyl ester (211), or benzoyl (212) were tolerated. The late-stage functionalization of pharmaceuticals containing a carboxylic acid functionality was successful and delivered corresponding monofluoroalkene derivative $\mathbf{2 1 3}$ in $51 \%$ yield.

The ring-fluorinated thiophene derivatives could be synthesized from $\mathrm{CF}_{3}$-cyclopropanes via $\mathrm{C}-\mathrm{F}$ bond activation under transition-metal-free conditions (Scheme 41), established by Ichikawa and co-workers [111]. With Lewis acid
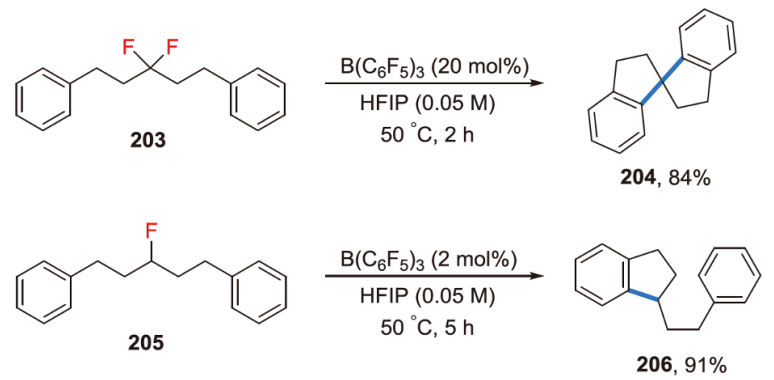

Scheme 38 Intramolecular Friedel-Crafts coupling of $\mathrm{C}\left(\mathrm{sp}^{3}\right)-\mathrm{F}$ bonds (color online).

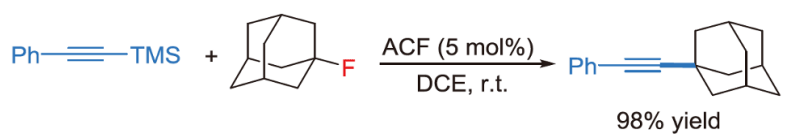

Scheme 39 ACF-catalyzed cross-coupling of silylalkynes with alkyl fluoride (color online).

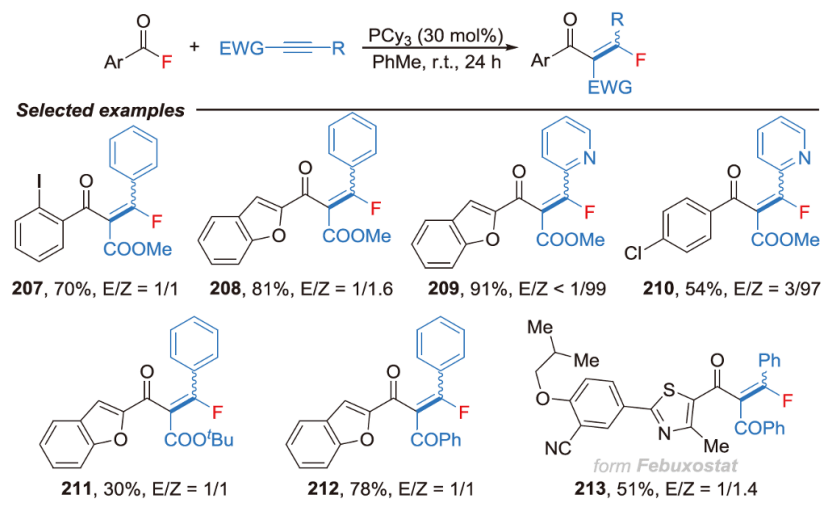

Scheme 40 Phosphine-catalyzed intermolecular acylfluorination of alkynes (color online). 
$\mathrm{Et}_{2} \mathrm{AlCl}$ as the promoter, the $\mathrm{CF}_{3}$-cyclopropanes eliminated $\mathrm{F}^{-}$to form $\mathrm{CF}_{2}$ cations, which were stabilized by the vinyl or cyclopropyl group. In the presence of thiocarboxylic acids or thiols, the $\mathrm{CF}_{2}$ cations underwent sulfanylation. They provided the products, which could be transformed to fluorodihydrothiophene (without $\mathrm{H}^{+}$) or difluorinated tetrahydrothiophenes $\left(\right.$ with $\mathrm{H}^{+}$) under alkaline conditions via 5-endotrig cyclization.

In an inspiring contribution, Wang and co-workers [63] recently reported the chemoselectivity-controllable $\mathrm{C}-\mathrm{F}$ functionalizations via sequential generation of difluoro- and monofluoroalkyl radicals (vide supra). The coupling of trifluoroacetamides with alkenes could be step by step (stage A or stage B) toward the synthesize of difluorinated or monofluorinated molecules (Scheme 42). This example showcases the potential of radical-involved $\mathrm{C}-\mathrm{F}$ bond activation to build up partially fluorinated complex molecules.

\section{Silyldefluorination}

Organosilanes are valuable synthetic intermediates and are

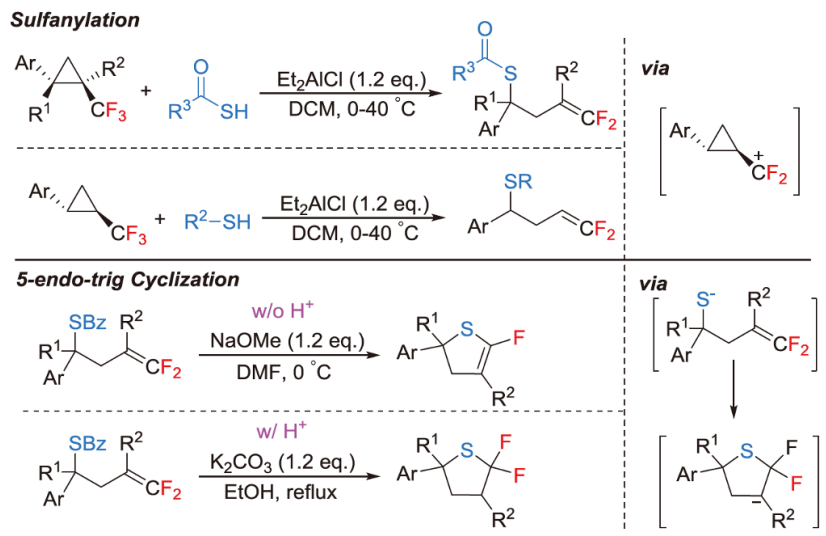

Scheme $41 \mathrm{C}-\mathrm{F}$ bond activation of $\mathrm{CF}_{3}$-cyclopropanes (color online).

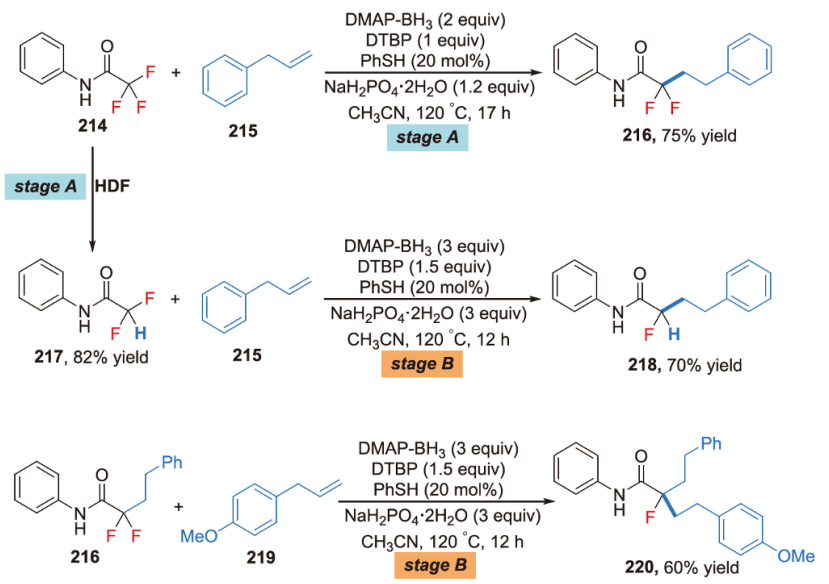

Scheme 42 Sequential C-F functionalization via spin-center shifts (color online). of great importance in medicine and materials science as well $[112,113]$. Compared with the classical methods for their synthesis which rely on stoichiometric aryl Grignard or aryl lithium compounds with chlorosilanes [114], greener protocols are highly desirable.

An early report by Roesky and co-workers [115] demonstrated that the Lewis base stabilized silylene (222, 224) reacted efficiently with polyfluoroarenes and delivered the corresponding $\mathrm{C}-\mathrm{Si}$ bond formation products (Scheme 43). Their subsequent research [116] found that these two silylene were also applicable to $\mathrm{PhN}=\mathrm{C}\left(\mathrm{CF}_{3}\right)_{2}$ (not shown). The discovery illustrates that the low valent silicon, a congener of carbon, mimics transition metal complexes.

A more general method of silyldefluorination of fluoroarenes was developed by Wgrthwein, Studer et al. [117] (Scheme 44). Utilized silyl lithium reagents, such as $\mathrm{PhMe}_{2} \mathrm{SiLi}, \mathrm{Ph}_{2}{ }^{t} \mathrm{BuSiLi}$, and $\mathrm{Ph}_{2} \mathrm{HSiLi}$, the fluoroarenes could be successfully converted into the corresponding silylated products (226-228). The non-activated fluorobenzene afforded dimethyldiphenylsilane (229). The electron-rich para-phenoxy derivative provided desired product 230 along with $15 \%$ of its meta isomer, which was formed via the corresponding aryne intermediate. The pyrrole-substituted fluorobenzene and para-fluoro-2-methyl-
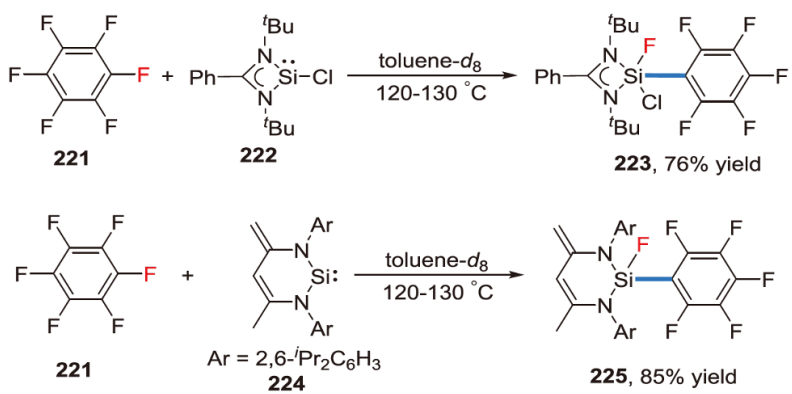

Scheme 43 C-Si bond formation with three-coordinate silylene (color online).

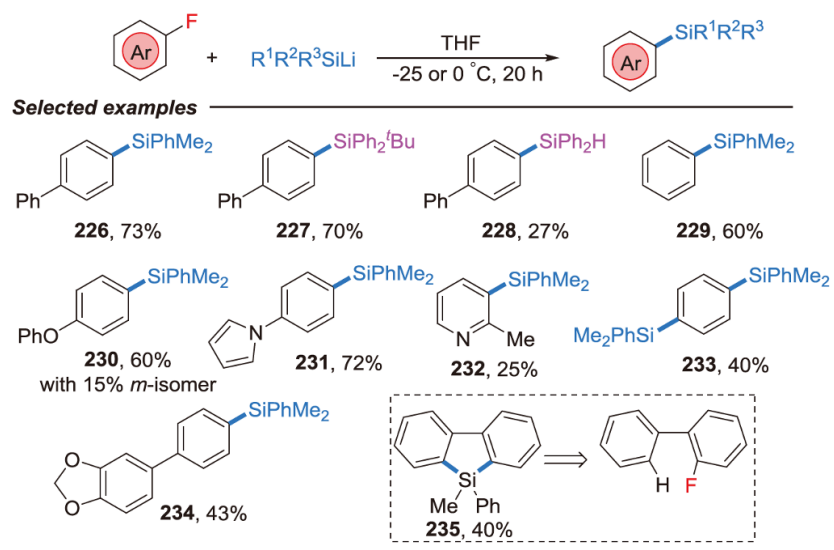

Scheme 44 Silyldefluorination of fluoroarenes by concerted $\mathrm{S}_{\mathrm{N}} \mathrm{Ar}$ (color online). 
pyridine delivered 23, 232 in $72 \%$ and $25 \%$ yield, respectively. It is worth noting that the ortho-fluorobiphenyl directly provided the 9-silafluorene $\mathbf{2 3 5}$ under the standard conditions. Unlike the classical nucleophilic aromatic substitution, this transformation also occurred on more electronrich aryl fluorides. Based on the experimental phenomena and DFT calculations, the authors proposed a concerted nucleophilic aromatic substitution mechanism.

Almost at the same time, Martin's group [118] reported a base-mediated defluorosilylation of $\mathrm{C}\left(\mathrm{sp}^{2}\right)-\mathrm{F}$ and $\mathrm{C}\left(\mathrm{sp}^{3}\right)-\mathrm{F}$ bonds (Scheme 45). The choice of $\mathrm{Et}_{3} \mathrm{SiBPin}$ offered the opportunity to functionalize the resulting aryl-Si bond selectively. Notably, no ortho- or meta-silyation products were even observed in the crude reaction mixtures (236), thus arguing against an aryne intermediates-attended process. The countercation $\mathrm{Li}^{+}$was critical for this transformation. With LiHMDS as the base, the defluorosilylation of unactivated $\mathrm{C}$ $\left(\mathrm{sp}^{2}\right)-\mathrm{F}$ bonds $(\mathbf{2 3 6}-\mathbf{2 4 0}), \mathrm{C}\left(\mathrm{sp}^{3}\right)-\mathrm{F}$ bonds (241-242), and the late-stage functionalization (243-244) performed well, constituting a complementary approach to existing $\mathrm{C}-\mathrm{Si}$ bond-forming protocols.

In industrially relevant research, Crimmin and co-workers [119] reported the silyldefluorination of the fluoroolefins HFO-1234yf, HFO-1234ze, and HFO-1336mzz (Scheme 46). The $\mathrm{PhMe}_{2} \mathrm{Si}-\mathrm{Li} \cdot \mathrm{THF}$ was synthesized by direct metalation of $\mathrm{PhMe}_{2} \mathrm{SiCl}$ with Li metal and it contains 1.5 THF molecules per silicon atom. Under mild conditions, this lithium silyl reagent reacted with $\mathrm{sp}^{2}$ or $\mathrm{sp}^{3} \mathrm{C}-\mathrm{F}$ bonds to deliver the fluorinated organosilanes in high yields with good selectivity. This route holds promise to be the means of upgrading and recycling fourth-generation refrigerants.

\section{Defluorinative $\mathrm{C}-\mathrm{N}$ bond formation}

Research on the catalytic formation of $\mathrm{C}-\mathrm{N}$ bonds has been

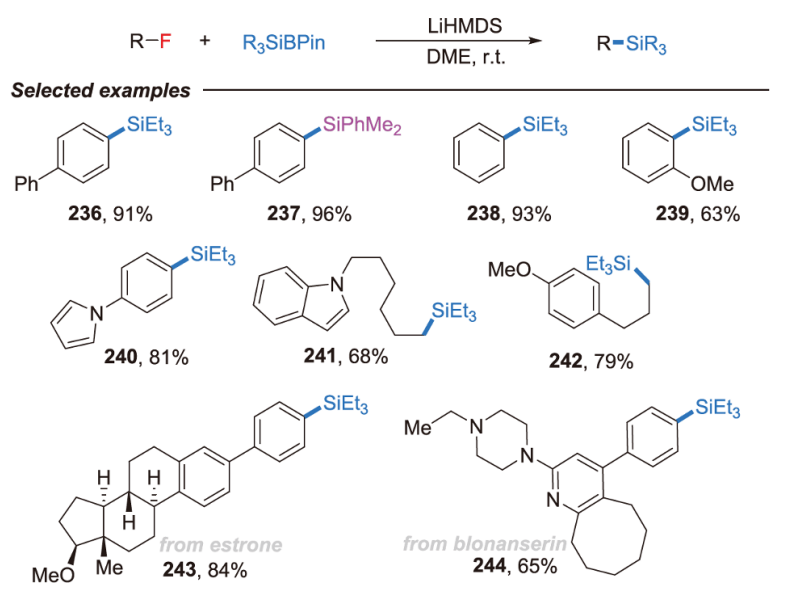

Scheme 45 Base-mediated defluorosilylation of $\mathrm{C}\left(\mathrm{sp}^{2}\right)-\mathrm{F}$ and $\mathrm{C}\left(\mathrm{sp}^{3}\right)-\mathrm{F}$ bonds (color online).

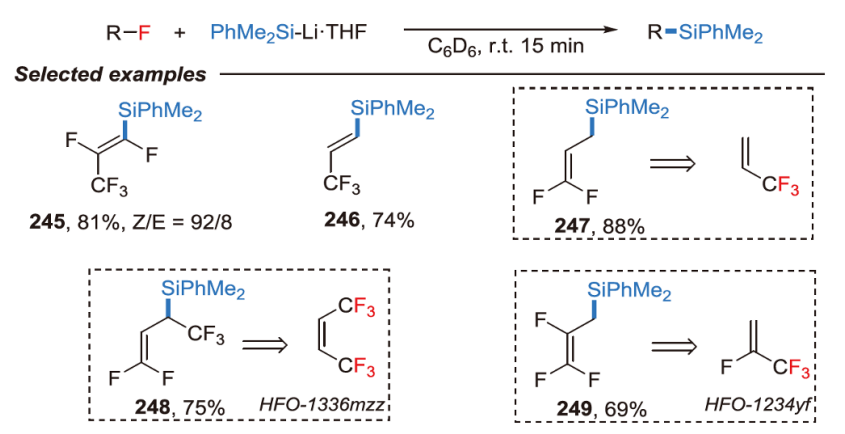

Scheme 46 Silyldefluorination of industrially relevant fluoroolefins (color online).

the topic with intensive studies [120]. The majority of C-N bond-forming transformations involve coupling aryl halides with nitrogen-containing compounds in the presence of a transition-metal catalyst, in which expensive catalysts and complicated ligands are usually required [121-125]. Despite a wealth of protocols for the formation of $\mathrm{C}-\mathrm{N}$ bonds that have been developed, including Ullmann, Chan-Lam and Buchwald-Hartwig reactions etc. [126-129], cleavage of C$\mathrm{F}$ bonds without the involvement of transition-metal to construct $\mathrm{C}-\mathrm{N}$ bonds is still challenging due to the stability of the $\mathrm{C}-\mathrm{F}$ bonds.

\subsection{Aromatic nucleophilic substitution reaction $\left(S_{N} A r\right)$}

The aromatic nucleophilic substitution reaction $\left(\mathrm{S}_{\mathrm{N}} \mathrm{Ar}\right)$ of aryl halides with aliphatic amines is a straightforward protocol for the preparation of aromatic amines [130,131]. Besides, this method has been applied in the synthesis of drugs and natural products as well. In 1997, MacDonald's group [132] utilized the diversomer technology to prepare Ciprofloxacin and several structurally related quinolones derivatives involving some $S_{N} A r$ of aryl fluorides with aliphatic amines (Scheme 47). And this is the first library of the quinolone antibacterial agents prepared by solid phase organic synthesis. First, the cyclization of the resin-bound enamide prepared by multi-step reaction in a solution of tetramethylquanidine (TMG) and dichloromethane (DCM) provided the corresponding resin-bound quinolones. One $\mathrm{C}-\mathrm{F}$ bond cleavage occurred, achieving aminodefluorination in the absence of transition-metal catalysis. The quinolones were then reacted with various solutions of piperazine compounds in $N$-methylpyrrolidinone (NMP). The crude products $(\mathbf{2 5 0}-\mathbf{2 5 5})$ were obtained with high purity via secondary $\mathrm{C}-\mathrm{N}$ bonding.

In addition, Seto and co-workers [133] reported a protocol for total synthesis of 7-\{4-[2-(butoxy)ethoxy]phenyl $\}-N-(4-$ $\{[$ methyl(tetrahydro-2 $H$-pyran-4-yl)amino]methyl $\}$ phenyl)1-propyl-2,3-dihydro-1H-1-benzazepine-4-carboxamide (259), an orally active CC chemokine receptor 5 (CCR5) antagonist (Scheme 48). The $\mathrm{S}_{\mathrm{N}} \mathrm{Ar}$ of aryl fluorides with 

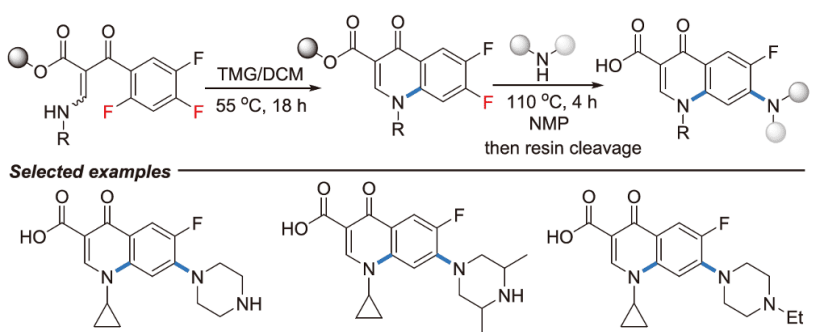

$250,13 \%$ yield; $96 \%$ purity
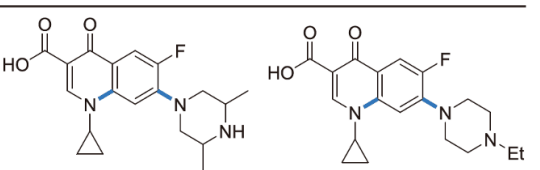

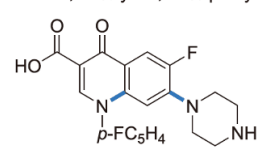

$253,6 \%$ yield; $86 \%$ purity
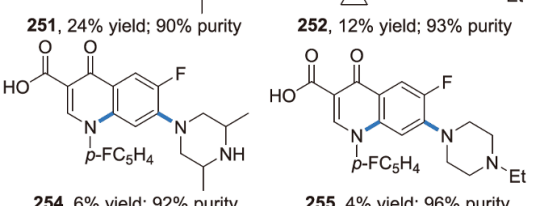

$255,4 \%$ yield; $96 \%$ purity
Scheme 47 A solid phase approach to quinolones using the diversomer technology (color online).

amines was involved in this transformation (Scheme 48, path 1). Detailly, the reaction of the 5-bromo-2-fluorobenzaldehyde $\mathbf{2 5 6}$ with amine $\mathbf{2 5 7}$ delivered the cyclization precursor 258 under basic conditions. Subsequently, compound 259 was prepared through a multi-step process. Furthermore, Ito's group [134] developed an alternative strategy of producing 259 using inexpensive materials (Scheme 48, path 2). Hydrolysis of 1-propylpyrrolidin-2-one $\left(\mathbf{2 5 8}^{\prime}\right)$ with $4 \mathrm{~N} \mathrm{NaOH}$ yielded 4-(propylamino)butanoic acid with high conversion. Then the reaction mixture was neutralized with concentrated $\mathrm{HCl}$ and sodium carbonate and 5-bromo-2fluorobenzaldehyde (256) was added. The resulting reaction mixture was refluxed to afford $\mathbf{2 5 8 ^ { \prime }}$ 'smoothly in a one-pot small-scale reaction. Eventually, the same product 259 could be obtained by a multi-step reaction as well.

With regard to fluoroquinolone antibacterial agents, in 2004, Choi and co-workers [135] disclosed a procedure for the synthesis of new fluoroquinolone antibacterial agents bearing pyrrolidine ring at the C-7 position (Scheme 49). Considering the importance of pyrrolidine ring in vitro antibacterial activities and pharmacokinetic profiles, optically inactive pyrrolidine derivative $\mathbf{2 6 0}$ was selected as the benchmark substrate to react with various fluoroquinolone compounds 261, 263, 265, 267 to provide the desired fluoroquinolone antibacterial agents 262, 264, 266, 268 in good to excellent yields by $\mathrm{S}_{\mathrm{N}} \mathrm{Ar}$.

In addition to the biologically active molecules mentioned above, various heterocyclic aromatic compounds, such as pyridines and quinolines are ubiquitous in natural products, pharmaceuticals, and pesticides [136-138]. There are several aminopyridine-based drug molecules, which are used to treat disorders. For the construction of such compounds, nucleophilic aromatic substitution reaction of 2-fluoropyridine with amines through $\mathrm{C}-\mathrm{F}$ bond activation is a straightforward and practical method. Singaram and co-workers [139] reported an efficacious lithium amides-promoted amination of 2fluoropyridine in the absence of transition-metal (Scheme

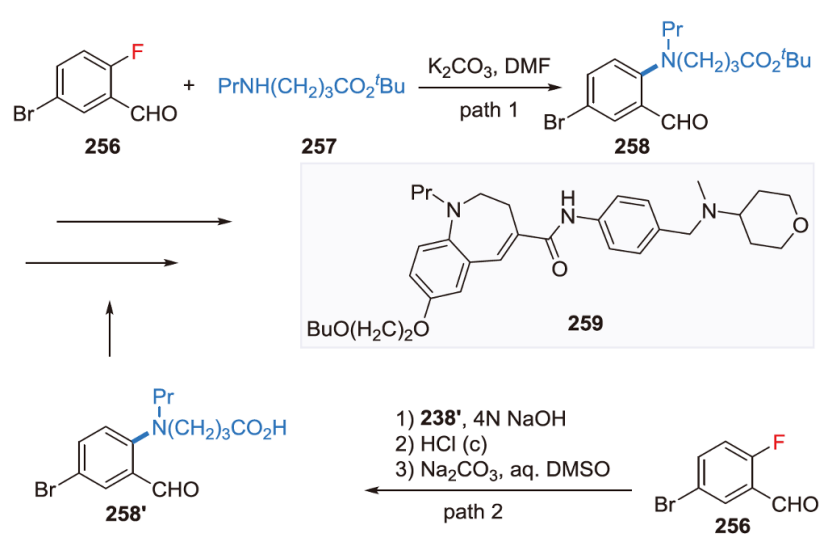

Scheme 48 Practical synthesis of an orally active CCR5 antagonist (color online).

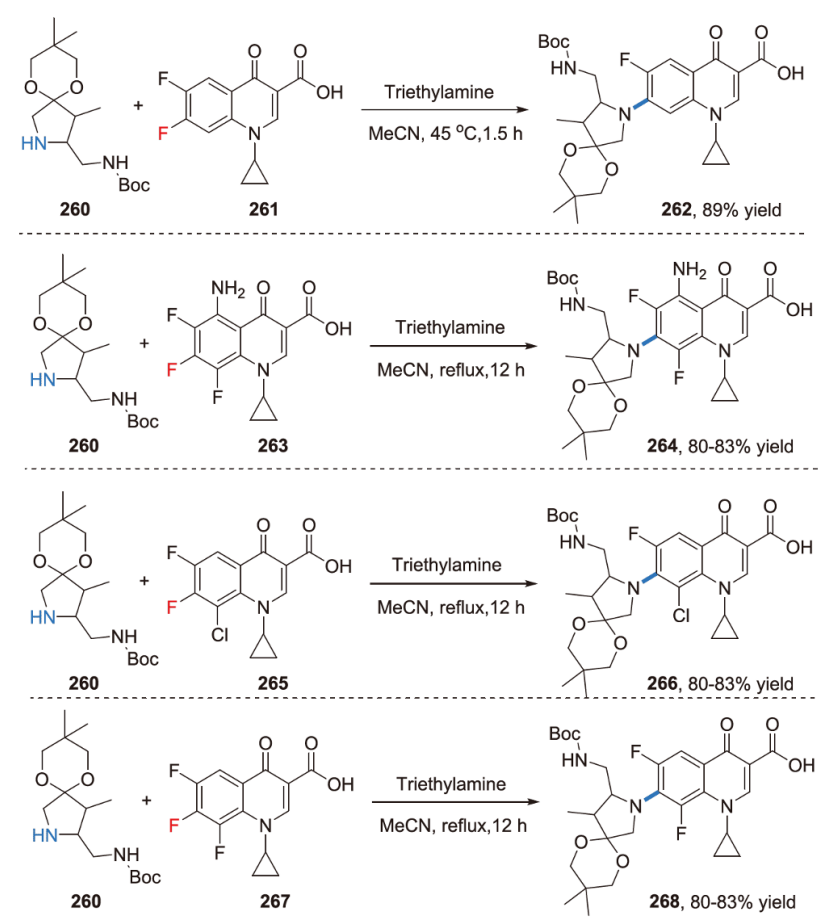

Scheme 49 Syntheses of new fluoroquinolone antibacterial agents via $\mathrm{C}-\mathrm{F}$ bond cleavage (color online).

50). A series of primary and secondary lithium amides could react with 2-fluoropyridine under mild reaction conditions to provide the corresponding aminopyridines (269-276) in moderate to good yields. Notably, this strategy could be successfully applied to synthesize enantiomerically pure aminopyridines (275), which might be utilized as potential ligands for asymmetric synthesis.

Additionally, Hevia and co-workers [140] reported an excellent methodology on amination of pyridine-containing fluoroarenes via magnesium-mediated $\mathrm{C}-\mathrm{F}$ bond activation (Scheme 51). Various $\beta$-diketiminate stabilized mononuclear Mg-containing amines complex as nucleophilic reacted with fluoroarenes under mild reaction conditions to obtain a series 

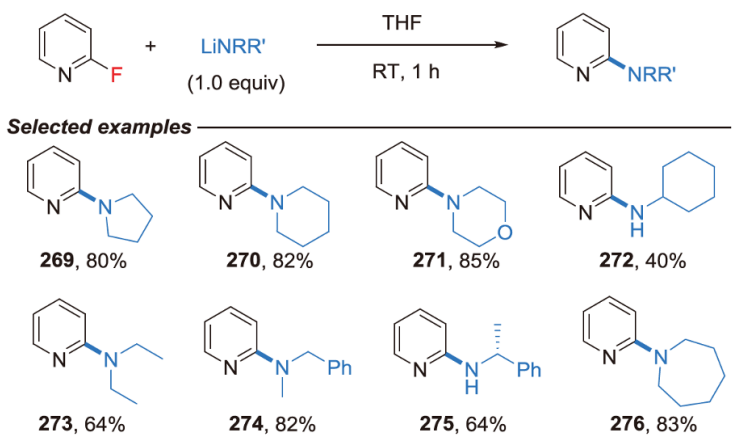

Scheme 50 Synthesis of aminopyridines from 2-fluoropyridine and lithium amides (color online).

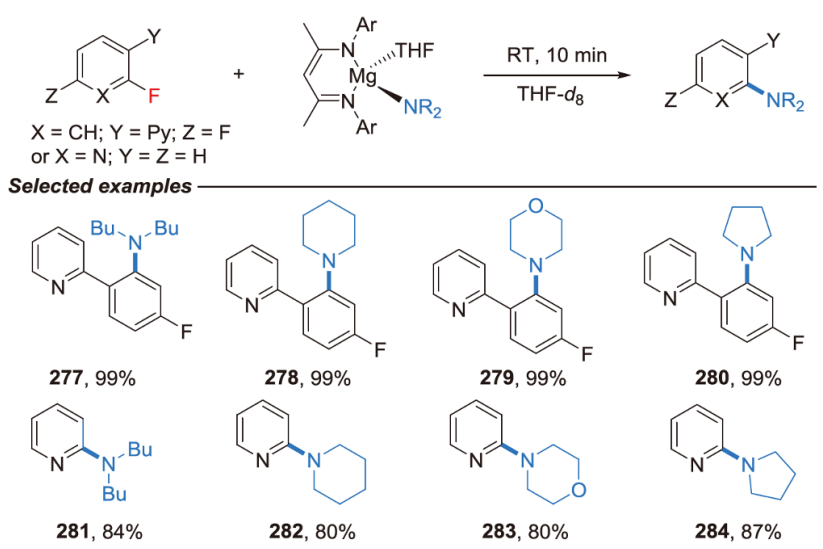

Scheme $51 \beta$-Diketiminate stabilized mononuclear Mg containing anines complexes promote $\mathrm{C}-\mathrm{F}$ bond cleavage (color online).

of aryl amines (277-284) in excellent yield. This reaction promotes the synthetic utility of $\beta$-diketiminate stabilized magnesium complexes and provides a novel route for aminodefluorination via $\mathrm{C}-\mathrm{F}$ bond activation of fluoroarenes.

Ding's group [141] presented a LiH-stimulated C-F bond activation for the formation of the $\mathrm{C}-\mathrm{N}$ bond (Scheme 52). Firstly, lithium-ion coordinates with the nitrogen atom of pyridine moiety. The $\mathrm{C}-\mathrm{F}$ bond would be slightly elongated owing to the $\mathrm{Li}$ ion and $\mathrm{F}$ atom interaction. Subsequently, adding an amine to the benzene ring would result in the formation of a $\mathrm{C}-\mathrm{N}$ bond. Finally, the cleavage of the $\mathrm{C}-\mathrm{F}$ bond in the Meisenheimer complex delivers the corresponding coupling product (285-296). This reaction features good substrate scope, high yields, mild reaction conditions as well as excellent regioselectivity for (difluorophenyl)pyridines.

In addition to aminopyridines, the aminoquinoline derivatives received much attention as important structural motifs in polyolefin catalyst families $[142,143]$. Transitionmetal catalysis is involved in the most known methods for the assembly of aminoquinolines [144-146]. And the transition-metal-free synthesis of aminoquinoline is still yet to be addressed. Taking advantage of the above same strategy,

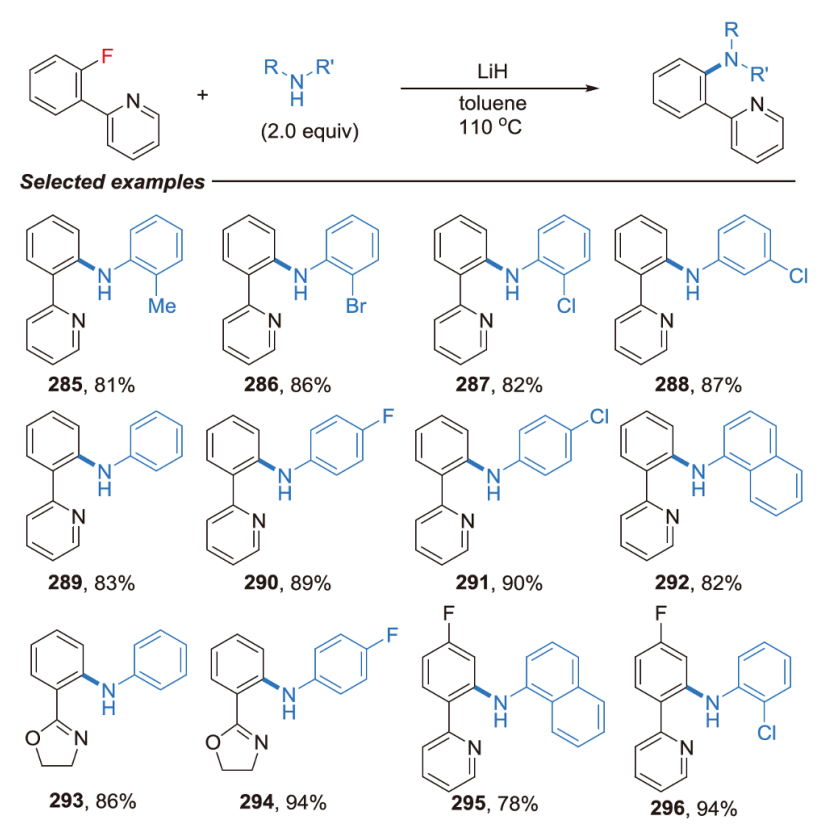

Scheme $52 \mathrm{~N}$-Heterocycle-assisted selective C-F bond cleavage through an $\mathrm{Li} / \mathrm{F}$ interaction (color online).

Ding and co-workers [147] also reported an efficient and general method for the selective synthesis of 8-aminoquinoline derivatives, in which $\mathrm{C}-\mathrm{F}$ bond on the 8 -substituted position was broken in the presence of lithium salts (Scheme 53), and excellent chemo- and regioselectivity was detected for polyfluoroquinolines. For the substrate scope, substituted amines, such as $o$-toluidine, 2,6-dimethylaniline, $p$-toluidine, as well as various polyfluoroquinolines presented good reactivity to generate the corresponding products (297-303) in moderate to good yields in the presence of $\mathrm{LiH}$. As for the reaction mechanism, firstly, lithium ion preferentially coordinates with the lone-pair electrons of the nitrogen atom. Meanwhile, the $\mathrm{C}-\mathrm{F}$ bond is activated and the anilide as nucleophile attacks the benzene ring, leading to the formation of the $\mathrm{C}-\mathrm{N}$ bond. Finally, the cleavage of the $\mathrm{C}-\mathrm{F}$ bond furnishes the targeted product along with releasing $\mathrm{LiF}$ (Scheme 53, bottom).

With respect to lithium-assisted $\mathrm{C}-\mathrm{F}$ bond activation, a novel tandem amination-reduction reaction has been reported by Singaram's group [148] (Scheme 54), in which 2( $\mathrm{N}, \mathrm{N}$-dialkylamino)-benzylamines are generated from 2halobenzonitriles and lithium $N, N$-dialkylaminoborohydride (LAB) reagents in the solution of THF. And the target products ( $N, N$-dialkylamino)benzylamine (305-308) could be easily obtained in good to excellent yields after a simple aqueous workup procedure. This process is believed to proceed through a tandem $\mathrm{S}_{\mathrm{N}} \mathrm{Ar}$ amination-reduction mechanism wherein the LAB reagent promotes the displacement of the fluorine substituents and the cyano groups. This one-pot procedure is an attractive synthetic tool for the nucleophilic aromatic substitution of halobesnzenes with less 

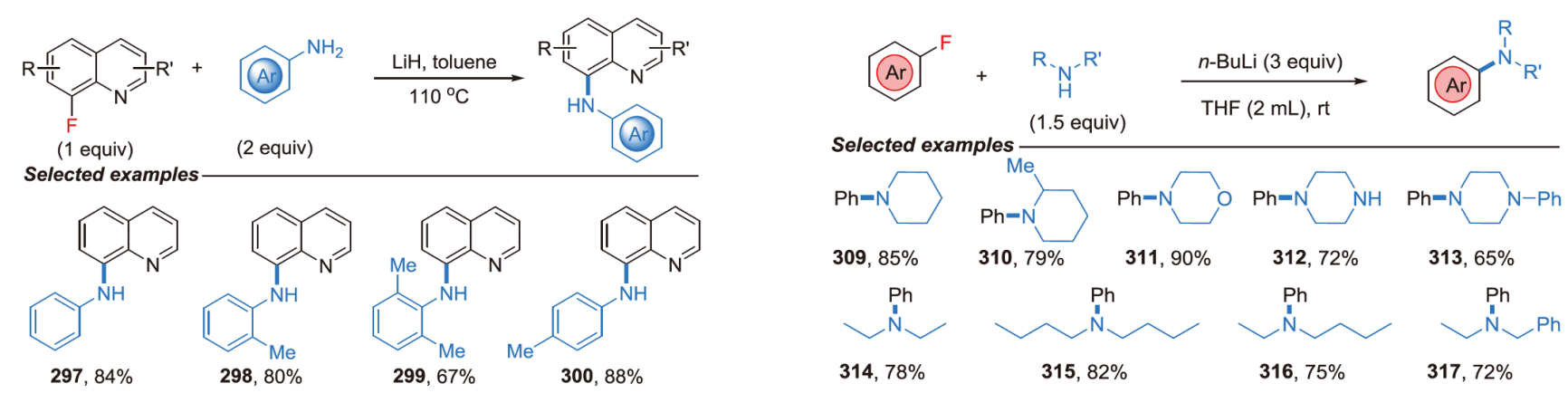
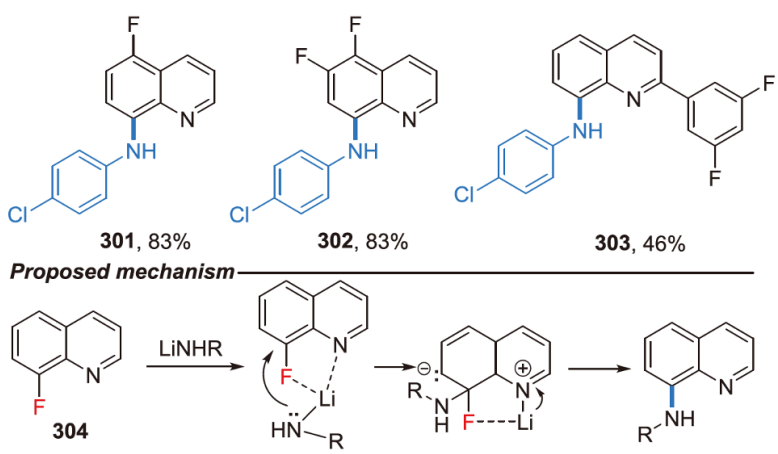

Scheme 53 Site-selective $\mathrm{C}-\mathrm{F}$ bond activation for synthesis of 8 aminoquinolines (color online).

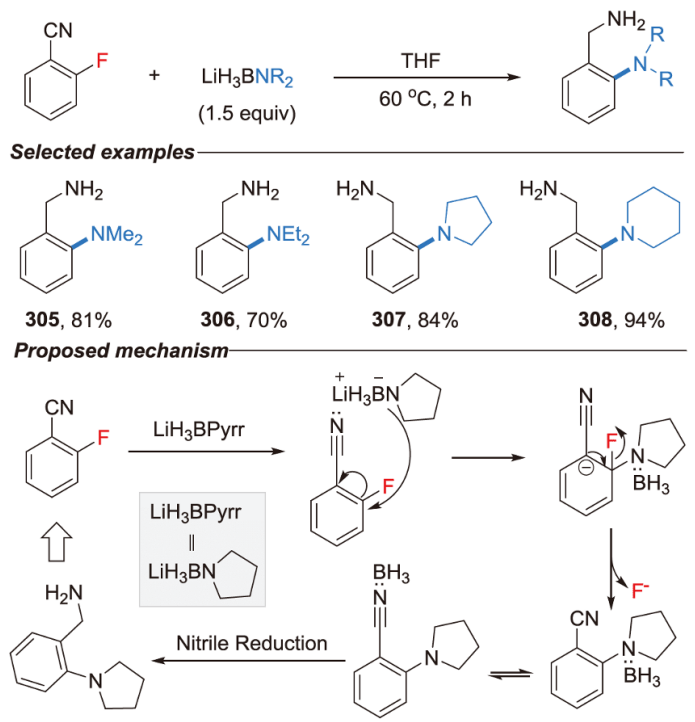

Scheme 54 Tandem $\mathrm{S}_{\mathrm{N}} \mathrm{Ar}$ amination-reduction reactions of 2-halobenzonitriles with lithium $N, N$-dialkylaminoborohydrides (color online).

nucleophilic amines.

Recently, Cao and co-workers [149] disclosed an efficient and mild strategy for the assembly of aromatic tertiary amines by the reactions of fluoroarenes with secondary amines in the presence of $n$-butyllithium at room temperature (Scheme 55). Both cyclic amines (piperidines, morpholines as well as piperazines) and acyclic amines (chain secondary amines) proceeded efficiently to afford a series of aromatic tertiary amines in good yields (309-317). This current
Scheme $55 n$-Butyllithium-mediated synthesis of $N$-aryl tertiary amines by reactions of fluoroarenes with secondary amines (color online).

methodology provides an alternative route to access valuable aromatic tertiary amines from readily available fluoroarenes.

For lithium salts-mediated $\mathrm{C}-\mathrm{F}$ bond activation, Diness and co-workers [150] reported the amination-defluorination of (ploy)fluorobenzenes enabling transition-metal-free $\mathrm{N}$ arylations of aliphatic amines (Scheme 56). The target products (318-325) were obtained in decent yields with high regio- and chemoselectivity. This reaction provided a prominent approach to forge polyfluorine-containing amines, enabling a valid transformation for late-stage functionalization of Vortioxetine.

Regio- and chemoselective amination of polyfluoroarenes has loomed as the popular and intriguing subject, beneficial to pharmaceuticals and agriculture chemistry [151-153]. Although the C-F bond activation of polyfluoroarenes has been flourishing, some challenges still exist for this type of transformation $[23,154,155]$. For example, selective monofunctionalization of polyfluorobenzene and preferentially activation of $\mathrm{C}-\mathrm{F}$ bond over other $\mathrm{C}-\mathrm{X}$ bonds $(\mathrm{X}=\mathrm{H}, \mathrm{Cl}, \mathrm{Br}$, I) are still not established. In addition to $\mathrm{C}-\mathrm{F}$ bond activation of polyfluoroarenes mentioned above, in 2015, Pang's group [156] developed a practical and effective aromatic nucleophilic monosubstitution reaction for the construction of various fluorine-containing aromatic amines (Scheme 57), in which selective cleavage of $\mathrm{C}-\mathrm{F}$ bond of various fluoroarenes (mono-, di-, tri-, tetra-, penta- and perfluorobenzene) proceeded under transition-metal free conditions. Fluoroarenes reacted with primary and secondary aromatic amines to generate target products in good to excellent yields in the presence of the base $(t-\mathrm{BuOK})$. In this $\mathrm{C}-\mathrm{F}$ bond activation, the solvents also controlled the reactivity of substrates. For instance, perfluorobenzene could work smoothly to render the desired products via selective $\mathrm{C}-\mathrm{F}$ bond cleavage by using THF as a reaction medium (326-329), whereas, other substrates could be effectively transformed into target products in good yields in DMSO (330-331) or toluene (332333).

Hevia's group [140] also developed selective cleavage of $\mathrm{C}-\mathrm{F}$ bond of perfluorobenzene under microwave radiation conditions, providing the expected products in higher yields 


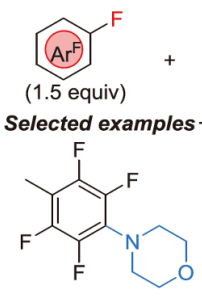

$318,87 \%$

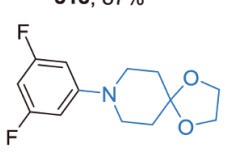

$322,76 \%$

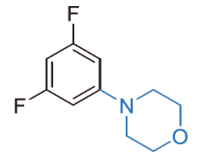

$319,76 \%$

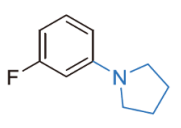

$323,80 \%$

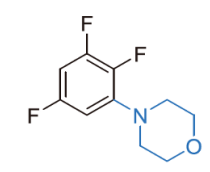

$320,70 \%$

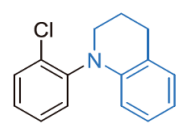

$324,74 \%$

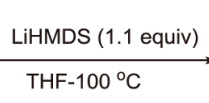

Scheme 56 Amination-defluorination obenzenes (color online).
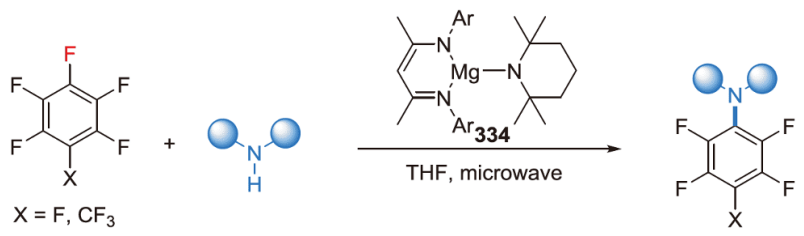

Selected examples

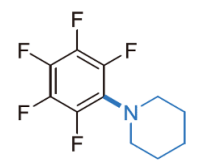

$335,65 \%$

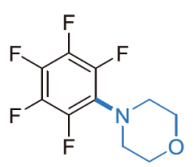

336, $67 \%$

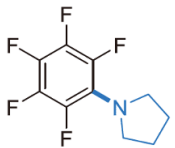

$337,73 \%$

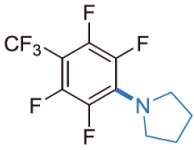

$338,91 \%$
Scheme 58 Microwave radiation promotes C-F bond activation (color online).

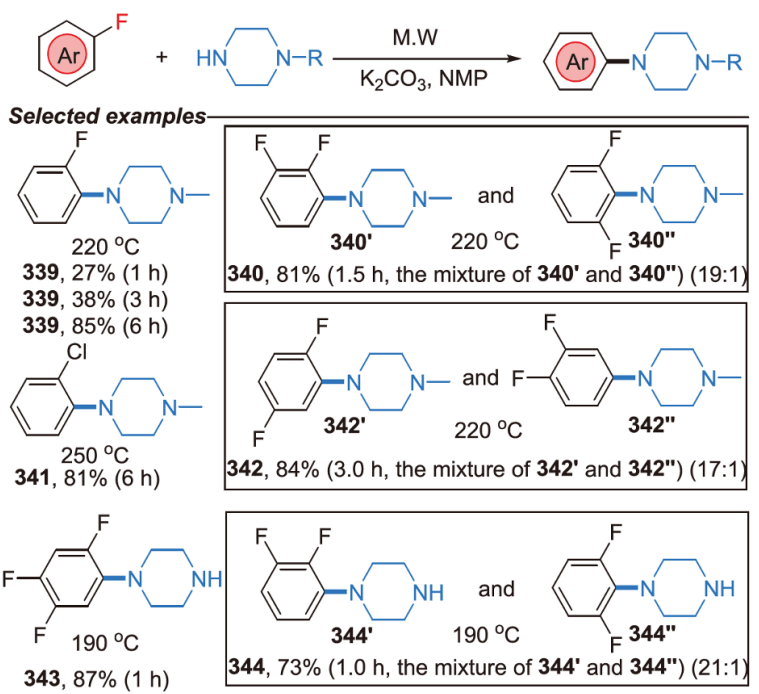

Scheme 59 Amination of fluorobenzenes via microwave irradiation (color online).

was employed as the starting material and reacted with hexafluorobenzene in the presence of an excess of $\mathrm{NaH}$ at $60{ }^{\circ} \mathrm{C}$, rendering the desired coupling product 347 in $24 \%$ yield (Eq. (1)). Subsequently, $N, N$-dihydroacenes 349, 351, 353 were isolated in modest yields using a similar reaction system (Eqs. (2-4)). This reaction allowed the rapid assembly of $\mathrm{N}, \mathrm{N}$-dihydrotetraazapentacene and -heptacene cores in a simple one-step process without transition-metal catalyst. And the targeted products of this transformation have great potential applications in material science.

Besides, Katz and co-workers [159] reported a transitionmetal free one-step synthetic route to afford benzodipyrroles via the successively cleavage of two $\mathrm{C}-\mathrm{F}$ bonds (Scheme 61). $p$-Toluidine was utilized as a nucleophile to react with ((4,6-difluoro-1,3-phenylene)bis(ethyne-2,1-diyl))dibenzene (354) to obtain final product $\mathbf{3 5 7}$ through compounds $\mathbf{3 5 5}$ and 356. This transformation involved two $S_{N} A r$ reactions, leading to a double substitution-anionic cyclization cascade and four $\mathrm{C}-\mathrm{N}$ bonds. 


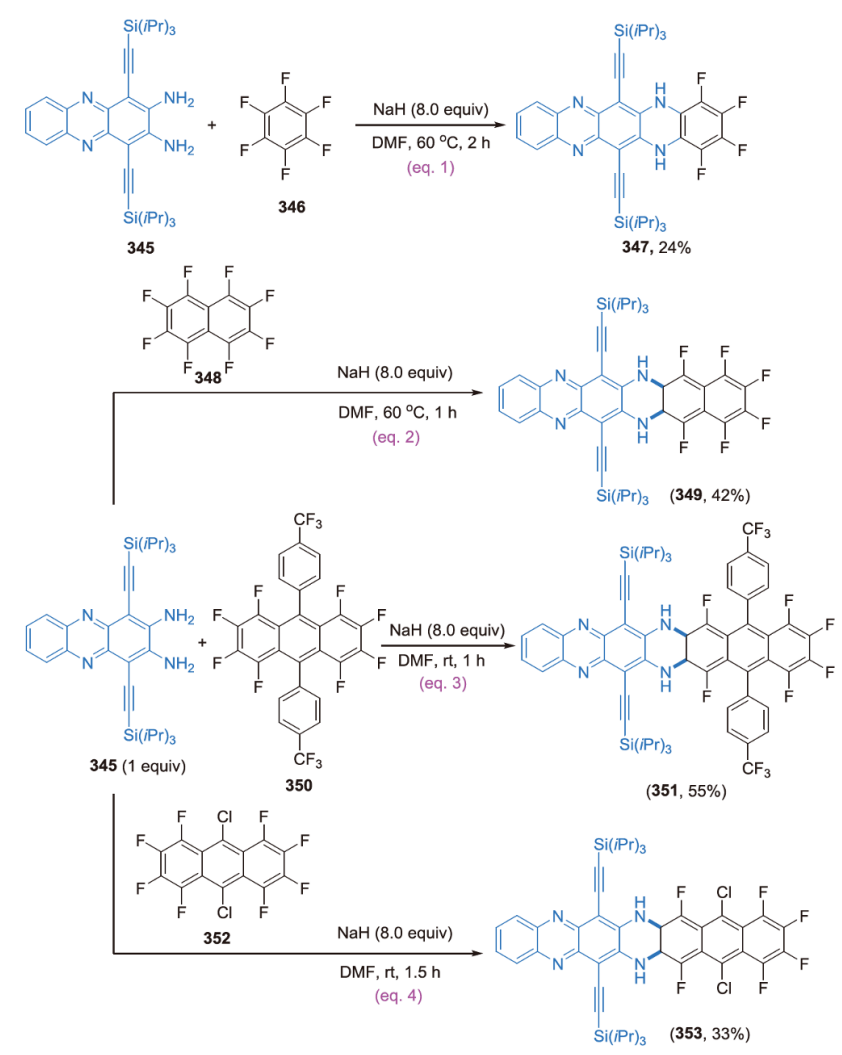

Scheme 60 Partially fluorinated tetraazaacenes by nucleophilic aromatic substitution (color online).

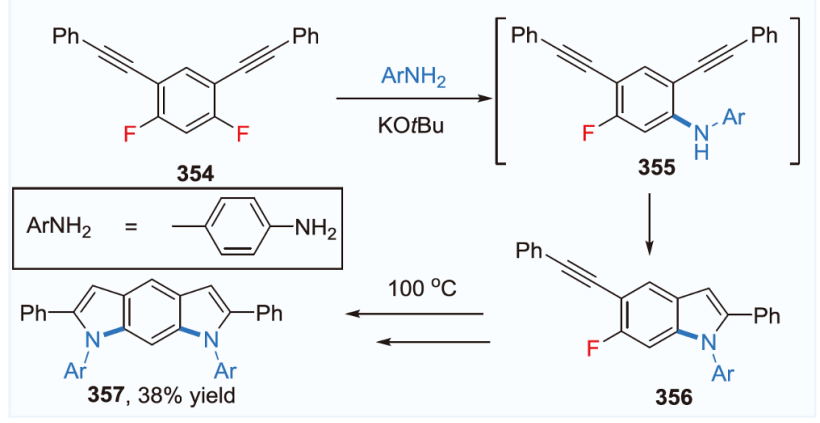

Scheme 61 Synthesis of benzodipyrroles via two C-F bond cleavage (color online).

$\mathrm{N}$-arylation of heteroaromatic compounds is an extremely significant $\mathrm{C}-\mathrm{N}$ bond formation reaction in organic synthesis. For nucleophilic aromatic substitution $\left(\mathrm{S}_{\mathrm{N}} \mathrm{Ar}\right)$ mentioned above, most nucleophilic reagents are aniline and aliphatic amines. In addition, there are some protocols for aromatic nitrogen-containing heterocycles as nucleophilic reagents achieving $\mathrm{C}-\mathrm{F}$ bond cleavage to form $\mathrm{C}-\mathrm{N}$ bond. $\mathrm{In}$ 2012, Diness and Fairlie [160] developed a valuable and very simple catalyst-free $N$-arylation of azole and indole derivatives, which involved direct $S_{N} A r$ of fluorine on unactivated benzene derivatives (Scheme 62). The authors investigated numerous experiments for reaction condition optimization, and then studied the substrate scope under microwave fea-

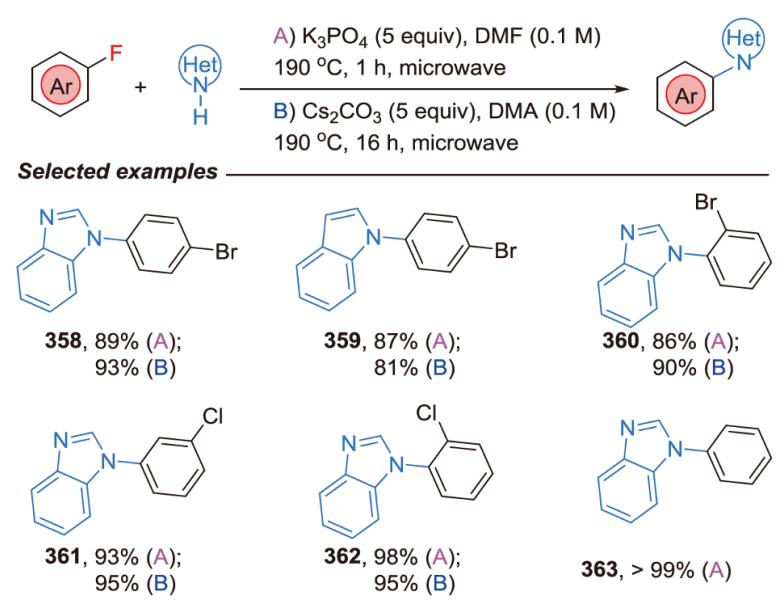

Scheme 62 Catalyst-free involved defluorinative amination of unactivated fluorobenzenes (color online).

turing high conversion of reactants and good tolerance of functional groups (bromo and chloro etc.). As a result, a range of fluorobenzene with various substituents reacted with indole or benzimidazole to afford the defluorinative amination products $(\mathbf{3 5 8}-\mathbf{3 6 3})$ in good to excellent yield in the absence of transition metal.

Recently, Lambert and co-workers [161] developed an electrophotocatalytic $\mathrm{S}_{\mathrm{N}} \mathrm{Ar}$ reaction of unactivated aryl fluorides at ambient temperature without transition-metal catalyst (Scheme 63). This reaction presents a nascent area for the combination of electrical energy and light to promote redox neutral reactions. For substrate scope, various $N$-heterocyclic compounds, such as pyrazole, triazoles, tetrazoles, bioactive molecule derivatives, could react with fluorobenzene to provide the target products (364-369) in moderate to good yields.

\subsection{Hydrogen bonds promote bimolecular nucleophilic substitution $\left(\mathrm{HB}-\mathrm{S}_{\mathrm{N}} 2\right)$}

As its known, fluorine atom can generate hydrogen bond with a hydrogen-bond donor (HBD) solvent, such as water or alcohols [162]. Numerous evidence suggests that the formation of hydrogen bond of organic fluorine is possible in an HBD solvent [163]. In this case, hydrogen bond would be considered as weak interaction of $\mathrm{C}-\mathrm{F}$ bond. During the last decades, hydrogen bond-accelerated cleavage of the $\mathrm{C}-\mathrm{F}$ bond has also drawn extensive attention from organic chemists. In 2013, Paquin and co-workers [163] reported a splendid water-stimulated $\mathrm{C}-\mathrm{F}$ bonds activation, in which alkyl fluorides were also amenable to undergo the substitution reactions via defluorinative amination (Scheme 64, Eq. (1)). DFT calculation reveals that C-F bond activation proceeds via stabilization of the transition state by a stronger hydrogen bond between alkyl fluorine and water, making the $\mathrm{C}-\mathrm{F}$ bond elongated. This finding presents a distinct and 


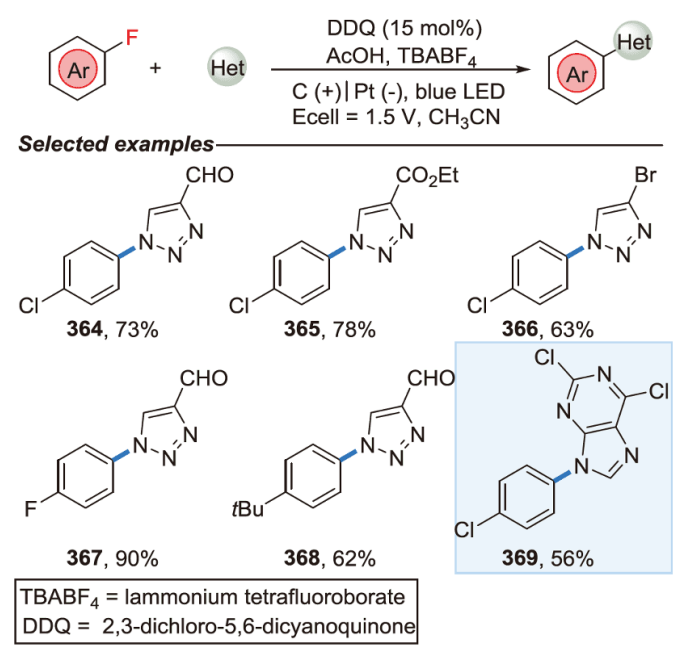

Scheme 63 Electrophotocatalytic $\mathrm{S}_{\mathrm{N}} \mathrm{Ar}$ reactions of unactivated aryl fluorides at ambient temperature and without transition metal (color online).

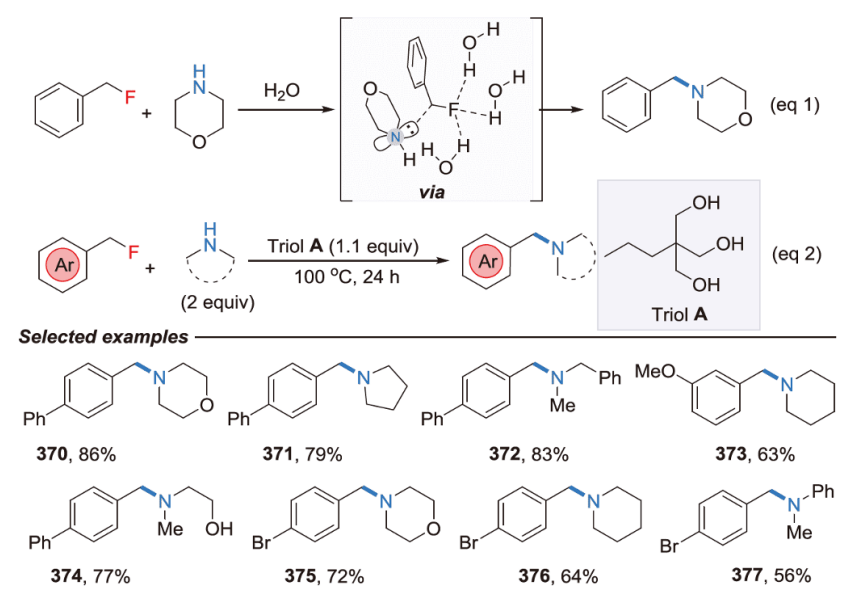

Scheme 64 Hydrogen bonding-promoted C-F bond cleavage to achieve $\mathrm{C}-\mathrm{N}$ bond (color online).

novel strategy for $\mathrm{C}-\mathrm{F}$ bond activation via hydrogen-bonding. Subsequently, the same group [164] reported a procedure using 1,1,1-tris(hydroxymethyl)propane as a hydrogen bond donating agent to achieve $\mathrm{C}-\mathrm{F}$ bond cleavage of benzylic fluorides (Scheme 64, Eq. (2)). The benzylic fluorides with various functional groups could react with secondary amines or anilines to generate benzylic amines (370-377) in $56 \%-86 \%$ yields. Mechanism investigations demonstrated that hydrogen bond-donating solvent promotes the $\mathrm{C}-\mathrm{F}$ bonds activation and hydrogen bond accepting one hinders the cleavage of $\mathrm{C}-\mathrm{F}$ bonds.

In 2014, Paquin's group [165] disclosed another hydrogen bond donating solvent promoted $\mathrm{C}-\mathrm{F}$ bond cleavage (Scheme 65). The reaction mechanism is proposed via DFT calculation and structure-activity analysis. Tricoordination of the triol with the fluorine-containing substrate is disfavored due to conformational constraints. However, the

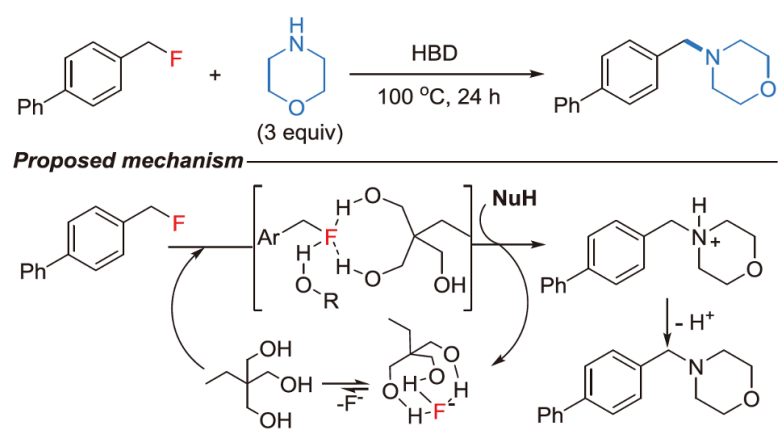

Scheme 65 Triol-promoted C-F bond cleavage (color online).

existence of three hydrogen bonds provides an optimal activation of the $\mathrm{S}_{\mathrm{N}} 2$ process. The author hypothesized that two $\mathrm{OH}$ groups from one triol molecule could coordinate with $\mathrm{F}$ atom and another $\mathrm{OH}$ group from a neighboring triol leads to the triad of activator. This transformation proceeded in the presence of morpholine under highly concentrated conditions.

Similarly, in 2018, O'Hagan's group [166] reported the enantioselective $\mathrm{C}-\mathrm{F}$ activation reaction using an enantiopure isotopomer of benzyl fluoride in a mixed solvent of isopropanol and water (Scheme 66). In this transformation, hydrogen bond promoted $\mathrm{C}-\mathrm{F}$ bond cleavage. Using water/ isopropanol as the activator afforded the benzylated products 379-381 in moderate yields. The ee values of the two products were very close to that of the original benzyl fluoride $((R)-1,95 \%)$, manifesting that a highly associative $\mathrm{S}_{\mathrm{N}} 2$-like route was involved. And the additional nucleophile must have approached on a coordinate anti to the $\mathrm{C}-\mathrm{F}$ bond leading to an inversion of the configuration. In addition, $N$ methylbenzylamine afforded a product $\mathbf{3 8 1}$ in $56 \%$ yields that did not resolve by ${ }^{2} \mathrm{H}$ NMR, so the ee value could not be determined.

\subsection{Other modes promote defluorinative amination/ azidation}

Although a number of methods have been disclosed for breaking $\mathrm{C}\left(\mathrm{sp}^{2}\right)-\mathrm{F}$ bonds in mono- and polyfluorinated aromatics as well as benzylic and allylic fluorides, the efficient cleavage of unactivated $\mathrm{C}\left(\mathrm{sp}^{3}\right)-\mathrm{F}$ bonds under transitionmetal-free conditions remains challenging [167-174]. As an alternative approach, Lewis acid could also prompt the $\mathrm{C}-\mathrm{F}$ bond activation. In 2016, Moran and co-workers [175] demonstrated an expedient $\mathrm{B}\left(\mathrm{C}_{6} \mathrm{~F}_{5}\right)_{3} \cdot \mathrm{H}_{2} \mathrm{O}$-promoted $\mathrm{C}-\mathrm{F}$ bond cleavage to achieve defluorinative azidation and defluorinative amination of tertiary aliphatic fluorides (Scheme 67). Trimethylsilyl azide $\left(\mathrm{TMSN}_{3}\right.$ ) was selected as the nitrogen source and nucleophile to form a new $\mathrm{C}-\mathrm{N}$ bond in this reaction. Subsequently, various tertiary aliphatic fluorides were tested under this reaction system. Homobenzylic 


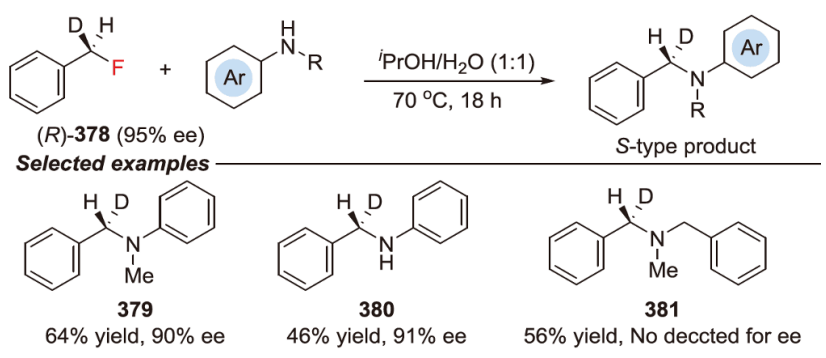

Scheme 66 Enantioselective C-F activation of enantiopure isotopomer of benzyl fluoride (color online).

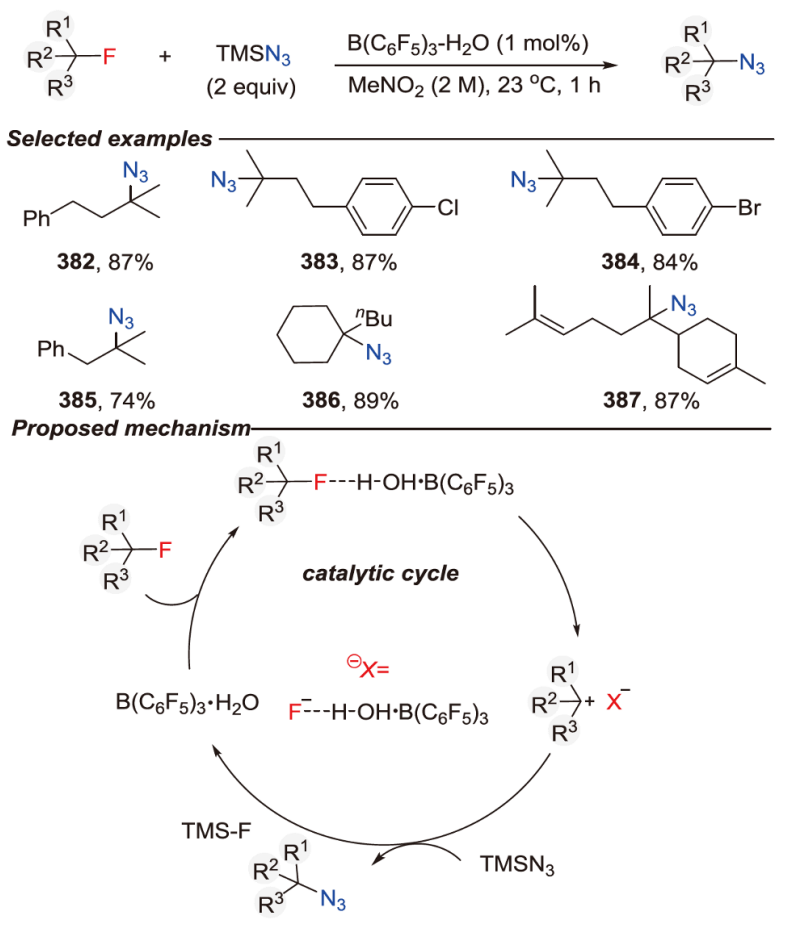

Scheme $67 \quad B\left(\mathrm{C}_{6} \mathrm{~F}_{5}\right)_{3} \cdot \mathrm{H}_{2} \mathrm{O}$-catalyzed defluorinative functionalization of tertiary aliphatic fluorides (color online).

tertiary aliphatic azides (381-386), cyclic aliphatic azide (386) as well as bisabolol-derived azide product (387) were smoothly prepared under the optimized conditions. Firstly, for the reaction mechanism, coordination of $\mathrm{B}\left(\mathrm{C}_{6} \mathrm{~F}_{5}\right)_{3} \cdot \mathrm{H}_{2} \mathrm{O}$ with tertiary aliphatic fluorides through hydrogen bond leads to the $\mathrm{C}-\mathrm{F}$ bond cleavage, generating tertiary aliphatic carbocation and fluoride anion. Then, the fluoride anion is trapped by silicon, providing the target products with decent yields.

For unactivated $\mathrm{C}\left(\mathrm{sp}^{3}\right)-\mathrm{F}$ bonds activation in the absence of transition metal, Terao and co-workers [176] reported a simple method for the cleavage of $\mathrm{C}\left(\mathrm{sp}^{3}\right)-\mathrm{F}$ bonds of alkyl fluorides to obtain various $\mathrm{C}\left(\mathrm{sp}^{3}\right)-\mathrm{X}(\mathrm{X}=\mathrm{Cl}, \mathrm{C}, \mathrm{H}, \mathrm{O}, \mathrm{S}, \mathrm{Se}$, $\mathrm{Te}, \mathrm{N}$ ) bonds using a hexane solution of organoaluminum reagents containing $\mathrm{Al}-\mathrm{X}$ bonds (Scheme 68 ). There was one example that 1-fluorooctane (388) reacted with (diethylamino)diisobutylaluminum (389) to afford aminodefluorination product 390 in $71 \%$ yield.
As its known, 1,4-naphthoquinone derivatives have properties of antioxidant, anti-malarial and so on [177]. Polyfluorinated functionalized 1,4-naphthoquinones could act as inhibitors for the growth of cancer cells [178]. Moreover, 1,4-benzoquinone derivatives exhibit diverse biological activity as well. Similar reactions of polyfluorinated benzoquinones were not investigated. In 2015, Selivanova and coworkers [179] reported the reaction of 2-X-trifluoro-1,4benzoquinones $(\mathrm{X}=\mathrm{F})$ with triphenylphosphane in various solvents (Scheme 69). An addition of a 0.6-equivalent of aniline 393 to a solution prepared by reaction of quinone 391 and $\mathrm{PPh}_{3}$ (1:1) gave [4-fluoro-2-oxido-5-oxo-3-(phenylamino)-6-(phenyl- imino) cyclohexa-1,3-dien-1-yl]triphenylphosphanium 394 in $29 \%$ yield (Eq. (1)). Two C-F bonds were cleaved and two different $\mathrm{C}-\mathrm{N}$ bonds were constructed (C-N single bond and $\mathrm{C}-\mathrm{N}$ double bond). Subsequently, An addition of a 0.6-equivalent of aniline 393 to a solution prepared by reaction of quinone 391 and $\mathrm{PPh}_{3}(1: 1)$ gave the intermediates 395. The addition of an excess of 4-phenoxyaniline 396 to the reaction mixture rendered a new compound \{4-fluoro-2-oxido-5-oxo-3-[(4-phenoxyphenyl)amino]6-(phenylimino)cyclohexa-1,3-dien-1-yl\} triphenylphosphanium (397, Eq. (2)). The addition of $\mathrm{H}_{2} \mathrm{O}$ to a solution prepared by reaction of quinone 391 and $\mathrm{PPh}_{3}(1: 1)$ afforded the intermediates 398, whereas, the addition of an excess of 4-phenoxyaniline 396 to the reaction mixture delivered another new compound \{4-fluoro-2-oxido-3,6-dioxo-5-[(4phenoxyphenyl)amino]cyclohexa-1,4-dien-1-yl $\}$ triphenylphosphanium (399, Eq. (3)).

Intramolecular cyclization reactions have provided a powerful platform to forge cyclic molecules, which are

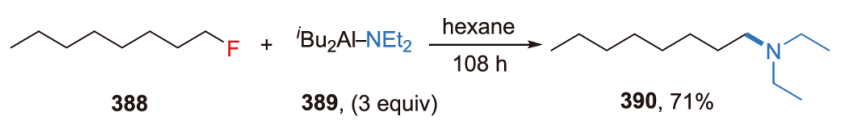

Scheme 68 Conversion of a $\mathrm{C}\left(\mathrm{sp}^{3}\right)-\mathrm{F}$ bond of alkyl fluorides to $\mathrm{C}\left(\mathrm{sp}^{3}\right)-\mathrm{N}$ bonds using organoaluminium reagents (color online).

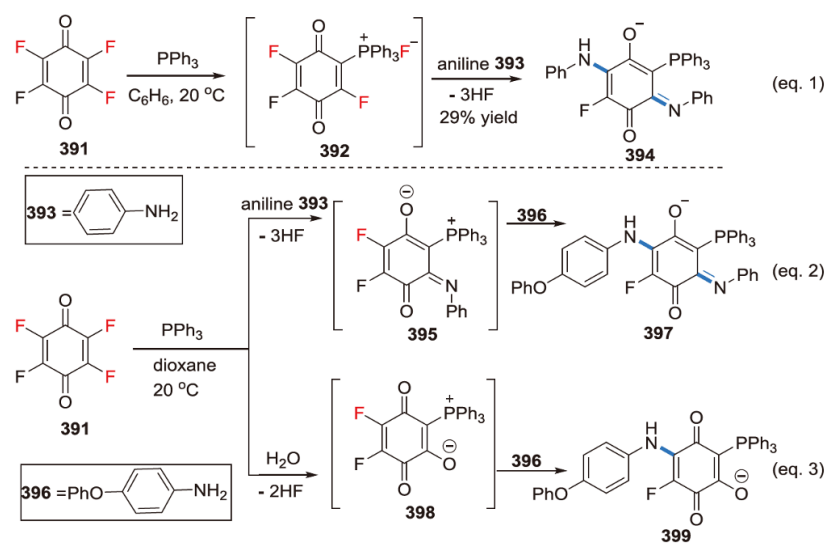

Scheme 69 Triphenylphosphanodefluorination of fluoranil and its derivatives (color online). 
widely present in natural products, pharmaceuticals, etc. Obviously, incorporating fluorine atoms into the target products could improve both the pharmacokinetics and membrane permeability compared with their non-fluorinated congeners [180-183]. Thus, fluorine-containing cyclization products are more significant. Intramolecular selective $\mathrm{C}-\mathrm{F}$ bond activation is a promising tactic for the construction of such compounds. In 2012, Shibata and co-workers [184] firstly disclosed the synthesis of a series of biologically relevant 3,5-diaryl-2-fluoromethyloxazolidin-2-ones scaffold by a conceptually new desymmetrization of unactivated aliphatic di-fluorides with silicon-induced catalytic C-F bond-cleavage as a key step to form $\mathrm{C}-\mathrm{N}$ bond (Scheme 70). Various 2-aryl-1,3-difluoromethyl-2-carbamates with a variety of aromatic ring substituents, such as methyl, fluoro, and chloro, were converted into cyclization products $\mathbf{( 4 0 0 -}$ 403) in good to excellent yields. For the reaction mechanism, firstly, BSA is activated by CsF to generate amido anion $\mathbf{A}$, along with the release of TMSF. Next, anion $\mathbf{A}$ abstracts a proton from reactant to yield the carbamate anion $\mathbf{B}$, along with the formation of $\mathrm{Me}_{3} \mathrm{SiNHCOMe}$. Subsequently, the $\mathrm{C}-\mathrm{F}$ bond of $\mathbf{B}$ is activated by interaction with the silicon atom of BSA, affording transition-state model $\mathbf{C}$ to induce intramolecular $\mathrm{S}_{\mathrm{N}}$ 2-like reaction, which furnishes the target product and TMSF. The amido anion $\mathbf{A}$, regenerated from $\mathbf{C}$, is the true catalyst in this catalytic cycle.

\section{Defluorinative borylation}

Organoborane compounds are ubiquitous in various organic transformations, which are significant synthetic inter-

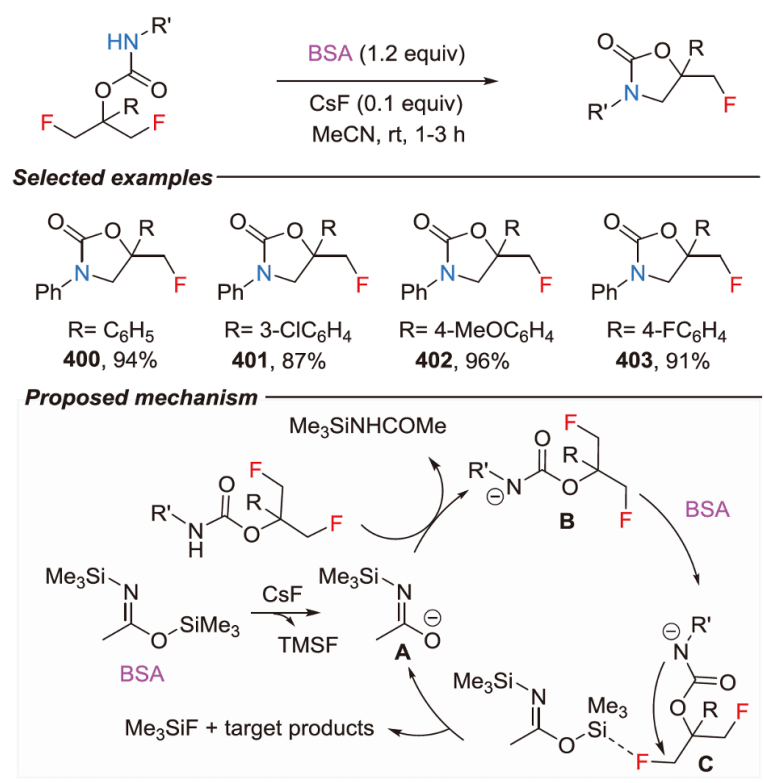

Scheme 70 Synthesis of fluoromethyl-3,5-diaryl-2-oxazolidinones by desymmetrization of 2-aryl-1,3-difluoropropan-2-ols (color online). mediates for the construction of pharmaceuticals, natural products, and organic materials [185-188]. Therefore, the development of simple and convenient methods for the assembly of organoborane compounds has received intensive attention [188]. Among them, direct borylation of nonactivated aromatic hydrocarbon compounds has become the subject of a wealth of landmark studies over the past several decades, as it represents a straightforward conversion from inert raw materials to value-added chemical building blocks. A battery of transition metals has been dominantly employed as a catalyst for defluorinative borylation of arenes [188]. Despite the transition-metal-catalyzed borylations, transition-metal-free borylation reactions have attracted ongoing interest. At present, photocatalysis has witnessed dramatic advances which have enabled previously inaccessible synthetic transformations for defluorinative borylation [189].

In 2016, Larionov and co-workers [190] reported a photoinduced borylation of electron-rich fluoroarenes via defluoration (Scheme 71). This light-induced defluorinative borylation proceeded smoothly under transition-metal-free and additive-free conditions with good functional group tolerance. 4-Fluoroaniline readily produced potassium (4aminophenyl)trifluoroborate (404) in 53\% yield, indicating that certain Ar-F bonds can be harnessed to construct boronic acids without transition metal. Similarly, various electronic-rich fluoroarenes could yield the target product (404-409) in moderate yields under the viable reaction conditions.

Soon after, Li's group [191] also developed a photocatalyzed C-B bond formation reaction via transition metalfree $\mathrm{C}-\mathrm{F}$ bond activation of electron-rich aryl fluorides (Scheme 72). But only three examples were given in this study, and notably, electron-rich aryl chlorides and phenol derivatives were suitable substrates in this reaction as well. 4-Fluorophenol, 4-fluoroaniline as well as 4-fluoro- $N, N$-dimethylaniline were utilized as substrates under continuousflow photolytic conditions, afforded the corresponding products (410-412) in 75\%,75\% and 53\% yields, respectively (Eqs. (1-3)).

Recently, Wu's group [192] developed a photo-mediated hydrogen atom transfer (HAT) process with $\mathrm{NHC}^{-\mathrm{BH}_{3}}$ (Scheme 73). The boryl radical was generated in-situ under mild conditions, which could react with polyfluoroarenes and gem-difluoroalkenes to produce synthetically valuable fluorinated organic boranes. When various polyfluoroarenes with different functional groups were employed as reactants, defluorinative borylation products $(\mathbf{4 1 5}-\mathbf{4 1 7})$ could be isolated in moderate to good yields.

Boryl radical reactions with polyfluoroarenes have been developed in the presence of photocatalysis [192,193]. However, polyfluoroarenes as radical acceptors have been rarely explored under photocatalysis-free conditions. Very recently, Taniguchi and co-workers [194] reported a thermal 


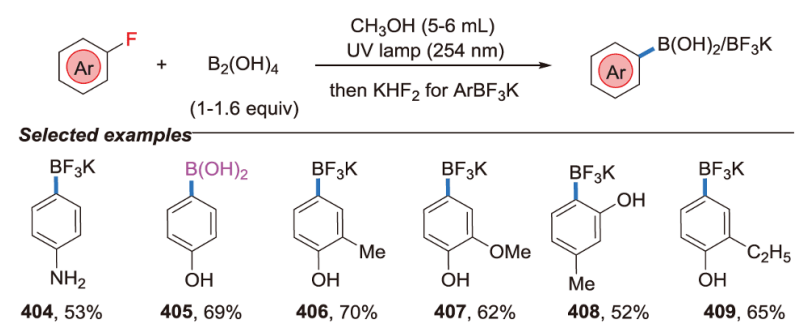

Scheme 71 Photoinduced borylation of electron-rich fluoroarenes via $\mathrm{C}-\mathrm{F}$ bond cleavage (color online).

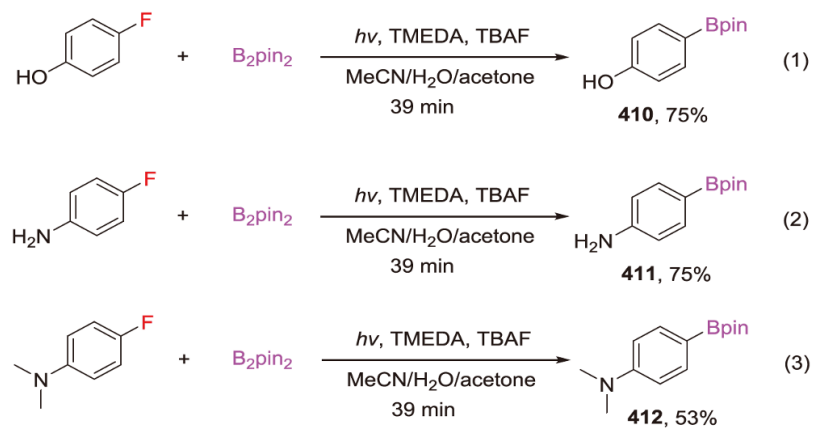

Scheme 72 Metal-free borylation of electron-rich aryl fluorides under continuous-flow photolytic conditions (color online).

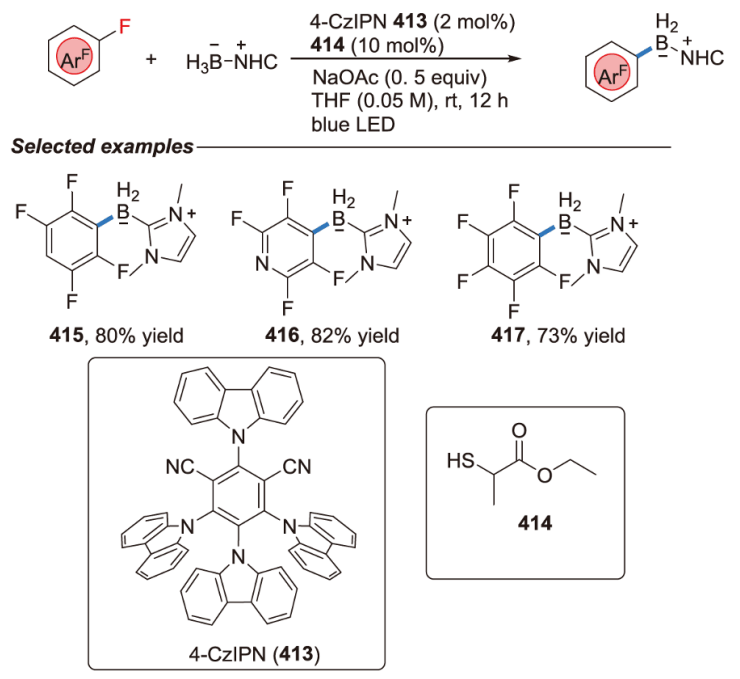

Scheme 73 Borylation of electron-rich aryl fluorides under continuousflow photolytic conditions (color online).

radical reaction of $\mathrm{N}$-heterocyclic carbene boranes (NHCboranes) by using polyfluoroarenes as good radical acceptors in the presence of di-tert-butyl peroxide (Scheme 74). In this process, the $\mathrm{C}-\mathrm{F}$ bond of polyfluoroarenes was substituted with the NHC-boryl group to obtain B-aryl NHC-borane derivatives (418-421). The current synthetic strategy could also be applied to forge new borane-containing liquid crystalline molecules with high thermal stability.

In addition to radical transformations of organoborane compounds to achieve defluorinative borylation, Finze and
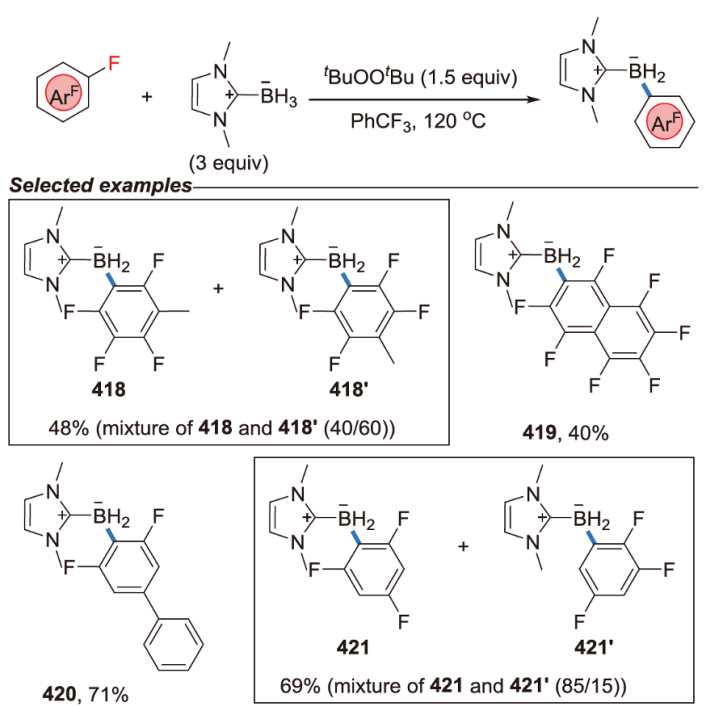

Scheme 74 Revisiting polyfluoroarenes as radical acceptors: radical C-F bond borylation of polyfluoroarenes with $N$-heterocyclic carbene boranes (color online).

co-workers [195] developed borylation of fluorinated arenes using the boron-centered nucleophile $\mathrm{B}(\mathrm{CN})_{3}{ }^{2-}$, a unique entry to aryltricyanoborates (Scheme 75 ). The potassium salt of the boron-centered nucleophile $\mathrm{B}(\mathrm{CN})_{3}{ }^{2-}$ readily reacted with perfluorinated arenes under mild reaction conditions, such as hexafluorobenzene, decafluorobiphenyl, octafluoronaphthalene and pentafluoropyridine, leading $\mathrm{KF}$ and the $\mathrm{K}^{+}$salts of the respective borate anions with one $\mathrm{B}(\mathrm{CN})_{3}$ unit bonded to the (hetero)arene. Pentafluorobenzenes R$\mathrm{C}_{6} \mathrm{~F}_{5}\left(\mathrm{R}=-\mathrm{CN},-\mathrm{OMe},-\mathrm{Me}\right.$, or $\left.-\mathrm{CF}_{3}\right)$ react with boroncentered nucleophile $\mathrm{B}(\mathrm{CN})_{3}{ }^{2-}$ obtained the corresponding products in good yields.

Tetracoordinate triarylboranes have a wide range of applications in organic photoelectronic materials due to their intriguing and unique properties. Song's group [187,196,197] has developed abundant protocols for accessing such compounds and has used such reagents to disclose various transformations. In 2019, Kinjo and co-workers [198] reported a metal-free selective borylation of arenes to afford tetracoordinate boranes via $\mathrm{C}-\mathrm{H} / \mathrm{C}-\mathrm{F}$ bond activation and dearomatization (Scheme 76). Intriguingly, when partially fluorinated arene derivatives were employed as the substrates, chemoselective $\mathrm{C}-\mathrm{F}$ bond activation was detected. Thus, the treatment of $\mathbf{4 2 2}$ with various fluorinated arene derivatives at $80{ }^{\circ} \mathrm{C}$ for $12 \mathrm{~h}$ afforded tetracoordinate boranes (423-425) in moderate to good yields.

\section{Other $\mathrm{C}-\mathrm{X}(\mathrm{X}=\mathrm{P}, \mathrm{O}, \mathrm{S}, \mathrm{Cl}, \mathrm{Br}, \mathrm{I})$ bonds formation reactions}

In addition to the above discussed examples, some innovative $\mathrm{C}-\mathrm{F}$ functionalization protocols such as $\mathrm{C}-\mathrm{P}$ bond, 


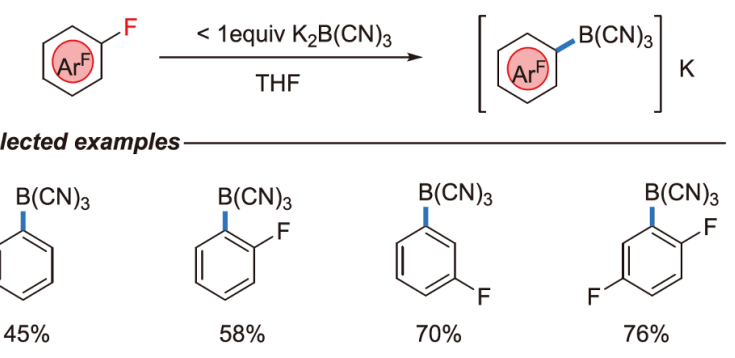

Scheme 75 Boron-centred nucleophile $\mathrm{B}(\mathrm{CN})_{3}{ }^{2-}$ promoted borylation of fluorinated arenes (color online).

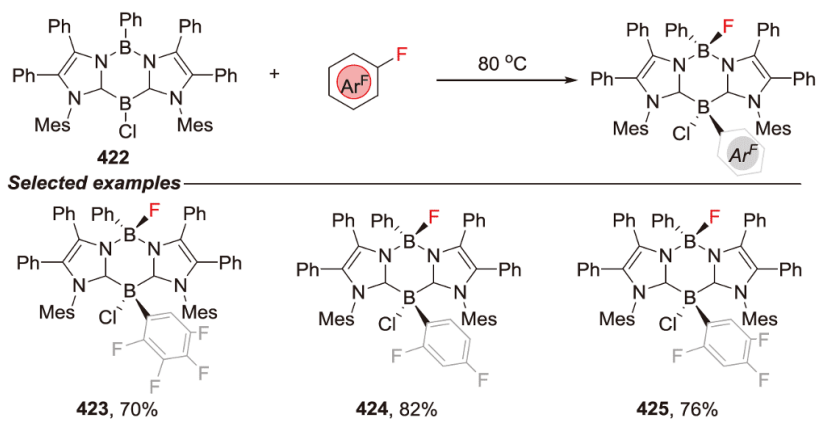

Scheme 76 Selective borylation of arenes by a diazadiborinine via $\mathrm{C}-\mathrm{H} /$ $\mathrm{C}-\mathrm{F}$ bond activation and dearomatization (color online).

$\mathrm{C}-\mathrm{O}$ bond, $\mathrm{C}-\mathrm{S}$ bond, and $\mathrm{C}-$ halogen $(\mathrm{Br}$ or $\mathrm{Cl})$ bond formation were also studied. The Lewis acids (via abstraction of fluorides) and appropriate bases (via nucleophilic substitution) were shown to activate the C-F bond successfully, thereby allowing for the coupling with nucleophiles or exchange with halogens.

Recently, Iwai and Sawamura et al. [199] reported a phosphinylation of non-activated aryl fluorides (Scheme 77). Employing LiHMDS as the base, most of the electronneutral and electron-rich aryl fluorides could participate in the reaction with substantially stabilized anionic P nucleophiles to form the corresponding tertiary phosphine oxides (426-431). However, fluorobenzene was not reactive under the conditions (429). The present protocol, noteworthy, applies to the synthesis of P-chiral tertiary phosphine oxides $(432,434)$. The quantum chemical calculations in the paper suggested a nucleophile-dependent mechanism that involves both concerted and stepwise $\mathrm{S}_{\mathrm{N}} \mathrm{Ar}$ reaction pathways.

Another successful $\mathrm{C}-\mathrm{P}$ bond formation protocol was developed by Young's group $[200,201]$. Utilizing $\mathrm{P}(o-\mathrm{Tol})_{3}$ and $\mathrm{B}\left(\mathrm{C}_{6} \mathrm{~F}_{5}\right)_{3}(\mathrm{BCF})$ as the frustrated Lewis pair, substrates bearing gem-difluoromethyl groups underwent highly selective monodefluorination, and the resulting products were subject to Wittig reaction protocols to provide a variety of monofluoroalkenes (Scheme 78, A). Based on this achievement, they subsequently developed a frustrating Lewispair-meditated selective single fluoride substitution in trifluoromethyl groups (Scheme 78, B). Treatment of trifluoromethyl arenes with 2,4,6-triphenylpyridine (TPPy) and

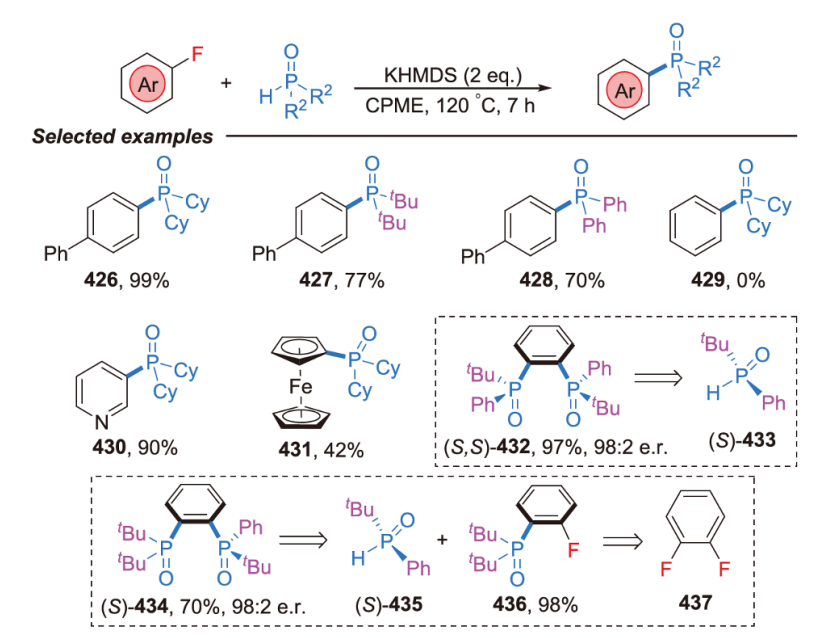

Scheme 77 Phosphinylation of non-activated aryl fluorides (color online).

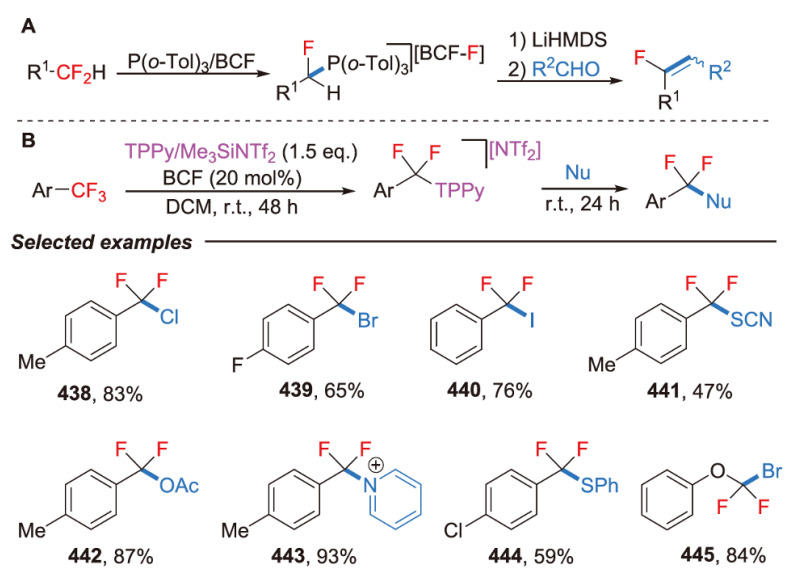

Scheme 78 Frustrated Lewis-pair-meditated selective single fluoride substitution of gem-difluoromethyl and trifluoromethyl groups (color online).

$\mathrm{Me}_{3} \mathrm{SiNTf}_{2}$ allowed for catalytic quantities of BCF (20 mol\%) to be used. In addition, the resulting pyridinium salts could be further functionalized via nucleophilic substitution, photoredox coupling, and electrophilic transfer reactions, delivering a range of difluoromethylene derivatives from such starting materials (438-445).

Based on the previous work (Scheme 70), Shibata's group [202] reported a phosphazene base-catalyzed $\mathrm{C}-\mathrm{O}$ bond formation to synthesize monofluoromethyl-substituted epoxides (Scheme 79). The proposed mechanism shows that the sterically demanding base $\mathbf{P}_{4}{ }^{-}{ }^{t} \mathbf{B u}$ first reacts with the alcohol 446 to afford reactive alkoxide ion pair $A$. Then, with the assistance of the silicon-based trapping reagent $\mathrm{N}(\mathrm{TMS})_{3}$, complex $\mathrm{B}$ provides the product $\mathbf{4 4 7}$ via $\mathrm{C}-\mathrm{F}$ bond cleavage and generates the true catalyst $\mathbf{C}$. The key to this catalytic cycle is the choice of the huge size superbase $\mathbf{P}_{4}{ }^{t} \mathbf{B u}$ $\left(500 \AA^{3}\right)$ [203], which prohibit the intermolecular silylation side reactions to silyl ethers. 


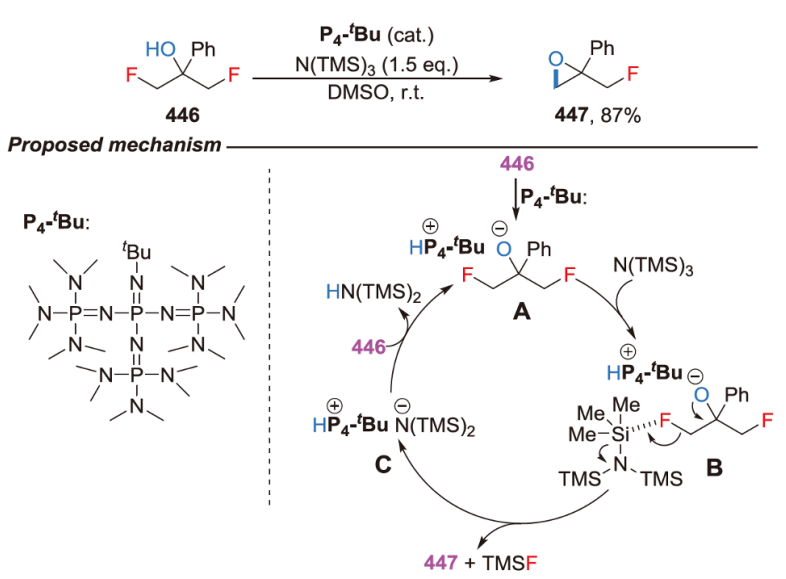

Scheme 79 Phosphazene base-catalyzed $\mathrm{C}-\mathrm{O}$ bond formation (color online).

The defluorinative $\mathrm{C}-\mathrm{Nu}$ bond formation reactions can also be achieved with Lewis acid, as demonstrated by Moran and co-workers [175] (Scheme 80). With $\mathrm{B}\left(\mathrm{C}_{6} \mathrm{~F}_{5}\right)_{3} \cdot \mathrm{H}_{2} \mathrm{O}$ as the catalyst, the tertiary aliphatic fluorides were efficiently converted into the corresponding $\mathrm{C}\left(\mathrm{sp}^{3}\right)-\mathrm{Nu}\left(\mathrm{Nu}=\mathrm{N}_{3}\right.$, SR, $\mathrm{OR}, \mathrm{NHR}$, and $\left.\mathrm{CH}_{2} \mathrm{R}\right)$ compounds $(\mathbf{4 4 8}-\mathbf{4 5 5})$. The protocol is remarkably general, and only alkyl fluorides reacted chemoselectively in the presence of other halides.

Zhu and Jiang's group [204] developed a defluorinative ipso-functionalization reaction of (trifluoromethyl)alkenes with oximes through an anionic $\mathrm{S}_{\mathrm{N}} 2$-type substitution pathway (Scheme 81$)$. Under the base conditions, various $O-(1,1-$ difluoroallyl)oxime ethers $(\mathbf{4 5 6}-\mathbf{4 6 1})$ were synthesized. The interesting aspect of this work is that the gem-difluoroalkene products it obtained contain two $\mathrm{C}\left(\mathrm{sp}^{3}\right)-\mathrm{F}$ bonds rather than $\mathrm{C}\left(\mathrm{sp}^{2}\right)-\mathrm{F}$ bonds.

Nucleophilic substitution reactions of pentafluoroarenes occurred firstly on the $\mathrm{C} 4$ position, then on the $\mathrm{C} 2 / \mathrm{C} 6$ and $\mathrm{C} 3 / \mathrm{C} 5$ positions if the nucleophilic reagents are overloading. This pattern was demonstrated by Ouyang and Yang's work [205]. As shown in Scheme 82, a DMF solution of sodium thiophenolate (462) and pentafluorobenzene (463) (1:1) were stirred overnight, generating 1-phenylthio-2,3,5,6-tetrafluorobenzene in $65 \%$ yield; the employment of excess $\mathbf{4 6 2}$, however, giving product 1,2,4-tris(phenylthio)-3,6-difluorobenzene (465).

The aluminum-based Lewis acids have shown to activate the $\mathrm{C}-\mathrm{F}$ bond (vide supra), and it can also be served as alkylation reagents to convert $\mathrm{C}\left(\mathrm{sp}^{3}\right)-\mathrm{F}$ bond into $\mathrm{C}-\mathrm{Nu}(\mathrm{Nu}$ $=\mathrm{Cl}, \mathrm{C}, \mathrm{H}, \mathrm{O}, \mathrm{S}, \mathrm{Se}, \mathrm{Te}, \mathrm{N}$ ) bonds by Terao's group [176] (Scheme 83). In the competitive experiments designed by the authors, only alkyl fluorides reacted chemoselectively in the presence of other alkyl halides.

In a similar manner, Young and co-workers [206] reported a catalytic halodefluorination of aliphatic $\mathrm{C}-\mathrm{F}$ bonds (Scheme 84). The halosilanes, in conjunction with aluminum catalysts, can convert alkyl fluorides into alkyl halides (466-

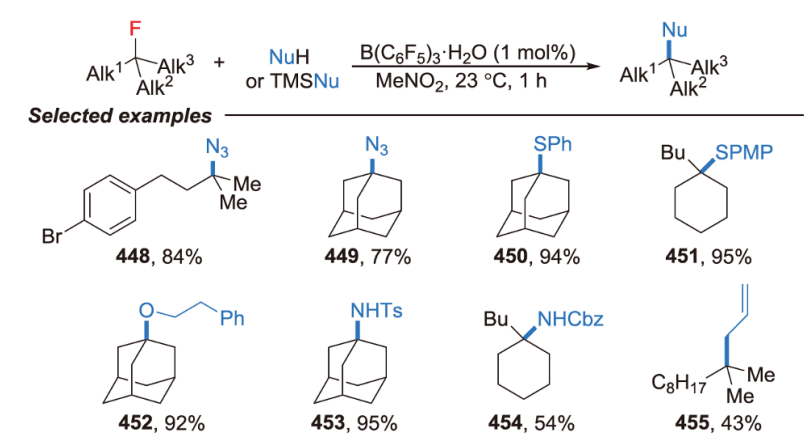

Scheme 80 Defluorinative functionalization of tertiary aliphatic fluorides (color online).

$$
\text { Selected examples }
$$

Scheme 81 Defluorooxylation of (trifluoromethyl)alkenes with oximes (color online).

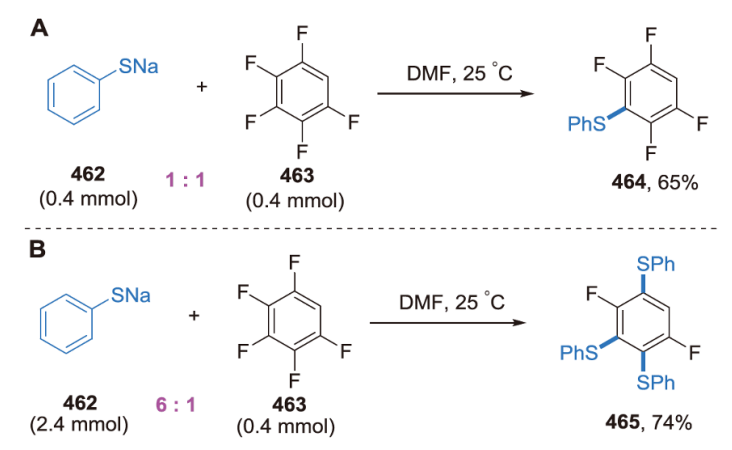

Scheme 82 Thiolation of pentafluorobenzene (color online).

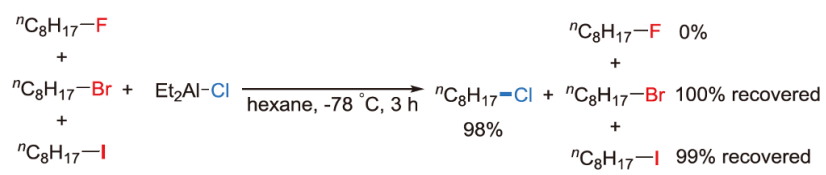

Scheme 83 Competitive reaction of alkyl halides with $\mathrm{Et}_{2} \mathrm{AlCl}$ (color online).

474) under mild conditions.

A $o$-hydrosilyl-assisted C-F transformation was developed by Yoshida's group [207] (Scheme 85). Utilizing in-situ generated trityl cation as the activator and the counter cation as the nucleophile, various transformations from $\mathrm{C}-\mathrm{F}$ to $\mathrm{C}-\mathrm{Cl}, \mathrm{C}-\mathrm{Br}, \mathrm{C}-\mathrm{OTs}$, and $\mathrm{C}-\mathrm{SCN}$ were achieved (475-480). 


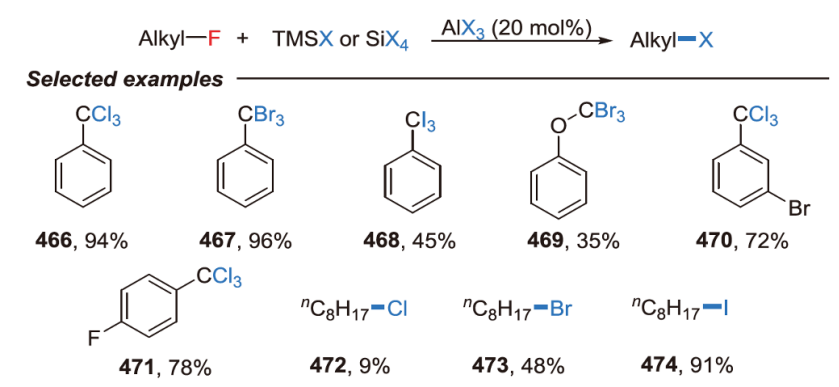

Scheme 84 Halodefluorination of aliphatic C-F bonds (color online).

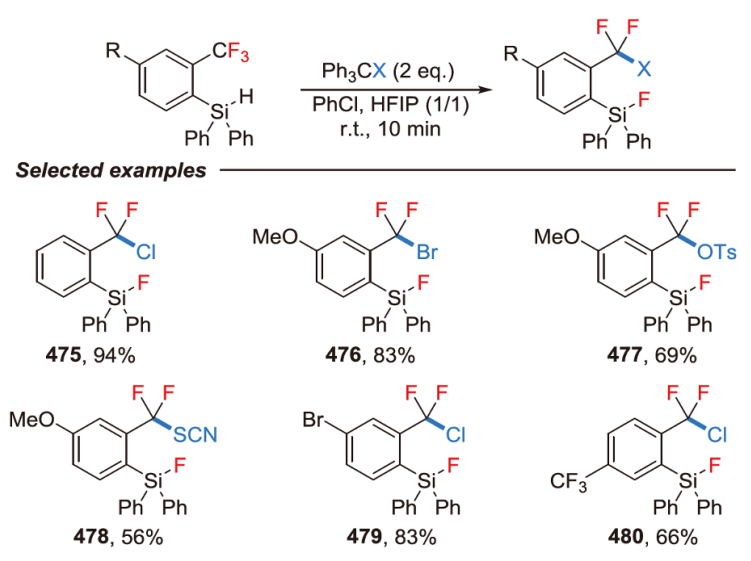

Scheme $85 o$-Hydrosilyl assisted C-F transformations (color online).

The $o$-hydrosilyl makes this transformation selectively (480). In addition to that, various ortho-functionalized difluorobenzyl chlorides and ortho-bromo-substituted difluorobenzyl chlorides were synthesized by the authors (not shown).

\section{Summary and outlooks}

Overall, the past decades has witnessed the significant advances in transition-metal-free $\mathrm{C}-\mathrm{F}$ bond activation of fluorine molecules. Most of the strategies used in the process are the capture of fluorine atoms with Lewis acid and the nucleophilic substitution reactions with strong bases. Some other approaches, such as the generation of radical intermediates by light or free radical initiators, have also been developed as alternatives to these strategies. There are also various types of reactions in which the $\mathrm{C}-\mathrm{F}$ bonds have been successfully converted into $\mathrm{C}-\mathrm{H}, \mathrm{C}-\mathrm{C}, \mathrm{C}-\mathrm{B}, \mathrm{C}-\mathrm{N}, \mathrm{C}-\mathrm{Si}, \mathrm{C}-$ $\mathrm{O}, \mathrm{C}-\mathrm{S}$ bonds etc. under transition-metal-free conditions. Meanwhile, research on selective $\mathrm{C}-\mathrm{F}$ bond activation is even more exciting and will certainly receive more attention in the near future.

The efficiency of these reactions is remarkable. However, from the substrate point of view, there is a high preference for $\mathrm{C}\left(\mathrm{sp}^{3}\right)-\mathrm{F}$ bonds. In addition to this, most of the articles published to date involving $\mathrm{C}\left(\mathrm{sp}^{2}\right)-\mathrm{F}$ bond are basic nucleophilic substitution reactions or activation of polyfluoroarenes, which are limited by the restricted substrates and/or harsh reaction conditions. This is attributed to the excessive BDE of the $\mathrm{C}\left(\mathrm{sp}^{2}\right)-\mathrm{F}$ bond. In this regard, light or electricity might be able to play a key role to achieve the expected breakthrough. Undoubtedly, selective C-F bond functionalization continues to be an attractive yet challenging topic. Selective activation of trifluoromethyl and polyfluorinated compounds remains one of the key research directions in this field. With continuing efforts, we expect to see the development of more useful approaches in this rapidly evolving research area.

\section{Conflict of interest The authors declare no conflict of interest.}

Open Access This article is distributed under the terms of the Creative Commons Attribution 4.0 International License (http://creativecommons. org/licenses/by/4.0/), which permits unrestricted use, distribution, and reproduction in any medium, provided you give appropriate credit to the original author(s) and the source, provide a link to the Creative Commons license, and indicate if changes were made.

1 Uneyama K. Organofluorine Chemistry. New York: John Wiley \& Sons, 2008

2 Gladysz JA, Curran DP, Horvath IT, Eds. Handbook of Fluorous Chemistry. Weinheim: Wiley-VCH, 2004

3 Kirsch P. Modern Fluoroorganic Chemistry: Synthesis, Reactivity, Applications. Weinheim: Wiley-VCH, 2004

4 Hiyama T. Organofluorine Compounds: Chemistry and Applications. Berlin: Springer, 2000

5 Purser S, Moore PR, Swallow S, Gouverneur V. Chem Soc Rev, 2008, 37: 320-330

6 Wang J, Sánchez-Roselló M, Aceña JL, del Pozo C, Sorochinsky AE, Fustero S, Soloshonok VA, Liu H. Chem Rev, 2014, 114: 2432-2506

7 Reichenbächer K, Süss HI, Hulliger J. Chem Soc Rev, 2005, 34: 22 30

8 O’Hagan D. Chem Soc Rev, 2008, 37: 308-319

9 Stahl T, Klare HFT, Oestreich M. ACS Catal, 2013, 3: 1578-1587

10 Arora A, Weaver JD. Acc Chem Res, 2016, 49: 2273-2283

11 Amii H, Uneyama K. Chem Rev, 2009, 109: 2119-2183

12 Hamel JD, Paquin JF. Chem Commun, 2018, 54: 10224-10239

13 Clot E, Eisenstein O, Jasim N, Macgregor SA, McGrady JE, Perutz RN. Acc Chem Res, 2011, 44: 333-348

14 Fujita T, Fuchibe K, Ichikawa J. Angew Chem Int Ed, 2019, 58: 390402

15 Eisenstein O, Milani J, Perutz RN. Chem Rev, 2017, 117: 8710-8753

16 Hunter L. Beilstein J Organ Chem, 2010, 6: 38

17 Ahrens T, Kohlmann J, Ahrens M, Braun T. Chem Rev, 2015, 115 : 931-972

18 Whittlesey MK, Peris E. ACS Catal, 2014, 4: 3152-3159

19 Shen Q, Huang YG, Liu C, Xiao JC, Chen QY, Guo Y. J Fluorine Chem, 2015, 179: 14-22

20 Liu C, Zhang B. Chem Record, 2016, 16: 667-687

21 Kameo H, Yamamoto H, Ikeda K, Isasa T, Sakaki S, Matsuzaka H, García-Rodeja Y, Miqueu K, Bourissou D. J Am Chem Soc, 2020, 142: 14039-14044

22 Senaweera S, Weaver JD. J Am Chem Soc, 2016, 138: 2520-2523

23 Choi J, Wang DY, Kundu S, Choliy Y, Emge TJ, Krogh-Jespersen K, 
Goldman AS. Science, 2011, 332: 1545-1548

24 Mo Z, Zhang Q, Deng L. Organometallics, 2012, 31: 6518-6521

25 Luo YR. Comprehensive Handbook of Chemical Bond Energies. Boca Raton: CRC press, 2007

26 Blanksby SJ, Ellison GB. Acc Chem Res, 2003, 36: 255-263

27 Lemal DM. J Org Chem, 2004, 69: 1-11

28 Ravishankara AR, Turnipseed AA, Jensen NR, Barone S, Mills M, Howard CJ, Solomon S. Science, 1994, 263: 71-75

29 Krause JR, Lampe FW. J Phys Chem, 1977, 81: 281-286

30 Scott VJ, Celenligil-Cetin R, Ozerov OV. J Am Chem Soc, 2005, 127: $2852-2853$

31 Douvris C, Ozerov OV. Science, 2008, 321: 1188-1190

32 Douvris C, Nagaraja CM, Chen CH, Foxman BM, Ozerov OV. J Am Chem Soc, 2010, 132: 4946-4953

33 Douvris C, Stoyanov ES, Tham FS, Reed CA. Chem Commun, 2007, 1145

34 Körbe S, Schreiber PJ, Michl J. Chem Rev, 2006, 106: 5208-5249

35 Panisch R, Bolte M, Müller T. J Am Chem Soc, 2006, 128: 96769682

36 Caputo CB, Stephan DW. Organometallics, 2012, 31: 27-30

37 Parks DJ, Blackwell JM, Piers WE. J Org Chem, 2000, 65: 30903098

38 Gevorgyan V, Rubin M, Benson S, Liu JX, Yamamoto Y. J Org Chem, 2000, 65: 6179-6186

39 Yang J, White PS, Schauer CK, Brookhart M. Angew Chem Int Ed, 2008, 47: 4141-4143

40 Stahl T, Klare HFT, Oestreich M. J Am Chem Soc, 2013, 135: 12481251

41 Chitnis SS, Krischer F, Stephan DW. Chem Eur J, 2018, 24: 65436546

42 Kikushima K, Grellier M, Ohashi M, Ogoshi S. Angew Chem Int Ed, 2017, 56: 16191-16196

43 Ahrens M, Scholz G, Braun T, Kemnitz E. Angew Chem Int Ed, 2013, 52: 5328-5332

44 Calvo B, Wuttke J, Braun T, Kemnitz E. ChemCatChem, 2016, 8: 1945-1950

45 Krahl T, Kemnitz E. J Fluorine Chem, 2006, 127: 663-678

46 Petrov VA, Krespan CG, Smart BE. J Fluorine Chem, 1996, 77: 139142

47 Krahl T, Stösser R, Kemnitz E, Scholz G, Feist M, Silly G, Buzaré JY. Inorg Chem, 2003, 42: 6474-6483

48 Klahn M, Fischer C, Spannenberg A, Rosenthal U, Krossing I. Tetrahedron Lett, 2007, 48: 8900-8903

$49 \mathrm{Gu}$ W, Haneline MR, Douvris C, Ozerov OV. J Am Chem Soc, 2009, 131: $11203-11212$

50 Jaeger AD, Ehm C, Lentz D. Chem Eur J, 2018, 24: 6769-6777

51 Phillips NA, White AJP, Crimmin MR. Adv Synth Catal, 2019, 361: 3351-3358

52 Meißner G, Kretschmar K, Braun T, Kemnitz E. Angew Chem Int Ed, 2017, 56: 16338-16341

53 Hayatifar A, Borrego A, Bosek D, Czarnecki M, Derocher G, Kuplicki A, Lytle E, Padilla J, Paroly C, Tubay G, Vyletel J, Weinert CS Chem Commun, 2019, 55: 10852-10855

54 Lu J, Khetrapal NS, Johnson JA, Zeng XC, Zhang J. J Am Chem Soc, 2016, 138: 15805-15808

55 Bauzá A, Mooibroek TJ, Frontera A. ChemPhysChem, 2015, 16 : 2496-2517

56 Vogt DB, Seath CP, Wang H, Jui NT. J Am Chem Soc, 2019, 141: 13203-13211

57 Sap JBI, Straathof NJW, Knauber T, Meyer CF, Médebielle M, Buglioni L, Genicot C, Trabanco AA, Noël T, Am Ende CW, Gouverneur V. J Am Chem Soc, 2020, 142: 9181-9187

58 Munoz SB, Ni C, Zhang Z, Wang F, Shao N, Mathew T, Olah GA, Prakash GKS. Eur J Org Chem, 2017, 2017(16): 2322-2326

59 Jaeger AD, Walter R, Ehm C, Lentz D. Chem Asian J, 2018, 13: 2908-2915

60 Arévalo A, Tlahuext-Aca A, Flores-Alamo M, García JJ. J Am Chem
Soc, 2014, 136: 4634-4639

61 Facundo AA, Arévalo A, Fundora-Galano G, Flores-Álamo M, Orgaz E, García JJ. New J Chem, 2019, 43: 6897-6908

62 Schoch TD, Mondal M, Weaver JD. Org Lett, 2021, 23: 1588-1593

63 Yu YJ, Zhang FL, Peng TY, Wang CL, Cheng J, Chen C, Houk KN, Wang YF. Science, 2021, 371: 1232-1240

64 Li J, Griffith WP, Davis I, Shin I, Wang J, Li F, Wang Y, Wherritt DJ, Liu A. Nat Chem Biol, 2018, 14: 853-860

65 Jin J, MacMillan DWC. Nature, 2015, 525: 87-90

66 Beckwith ALJ, Crich D, Duggan PJ, Yao Q. Chem Rev, 1997, 97: 3273-3312

67 Kiso Y, Tamao K, Kumada M. J Organomet Chem, 1973, 50: C12C14

68 Xiong Y, Wu J, Xiao S, Xiao J, Cao S. J Org Chem, 2013, 78: 45994603

69 Dai W, Shi H, Zhao X, Cao S. Org Lett, 2016, 18: 4284-4287

70 Lu F, Sun H, Du A, Feng L, Li X. Org Lett, 2014, 16: 772-775

71 Sun Y, Sun H, Jia J, Du A, Li X. Organometallics, 2014, 33: 10791081

72 Dutta T, Woody KB, Watson MD. J Am Chem Soc, 2008, 130: 452453

73 Wang Y, Watson MD. J Am Chem Soc, 2006, 128: 2536-2537

74 Sanji T, Motoshige A, Komiyama H, Kakinuma J, Ushikubo R, Watanabe S, Iyoda T. Chem Sci, 2015, 6: 492-496

75 Jin G, Zhang X, Cao S. Org Lett, 2013, 15: 3114-3117

76 Ji X, Huang T, Wu W, Liang F, Cao S. Org Lett, 2015, 17: 50965099

77 Liu C, Zhao H, Zhao H, Wang Z, Zhang B. RSC Adv, 2015, 5: 31993-31997

78 Shigeno M, Okawa T, Imamatsu M, Nozawa-Kumada K, Kondo Y. Chem Eur J, 2019, 25: 10294-10297

79 Allemann O, Duttwyler S, Romanato P, Baldridge KK, Siegel JS. Science, 2011, 332: 574-577

80 Allemann O, Baldridge KK, Siegel JS. Org Chem Front, 2015, 2: 1018-1021

81 Amsharov KY, Kabdulov MA, Jansen M. Angew Chem Int Ed, 2012, 51: 4594-4597

82 Amsharov KY, Merz P. J Org Chem, 2012, 77: 5445-5448

83 Steiner AK, Amsharov KY. Angew Chem Int Ed, 2017, 56: 1473214736

84 Akhmetov V, Feofanov M, Troyanov S, Amsharov K. Chem Eur J, 2019, 25: 7607-7612

85 Suzuki N, Fujita T, Amsharov KY, Ichikawa J. Chem Commun, 2016, 52: 12948-12951

86 Shao B, Bagdasarian AL, Popov S, Nelson HM. Science, 2017, 355: 1403-1407

87 Zhu J, Pérez M, Caputo CB, Stephan DW. Angew Chem Int Ed, 2016, 55: 1417-1421

88 Ikeda M, Matsuzawa T, Morita T, Hosoya T, Yoshida S. Chem Eur J, 2020, 26: 12333-12337

89 Theodoridis G. Tetrahedron Lett, 1998, 39: 9365-9368

90 Prakash GKS, Hu J, Simon J, Bellew DR, Olah GA. J Fluorine Chem, 2004, 125: 595-601

91 Yoshida S, Shimomori K, Kim Y, Hosoya T. Angew Chem Int Ed, 2016, 55: 10406-10409

92 Luo C, Bandar JS. J Am Chem Soc, 2019, 141: 14120-14125

93 Chitnis SS, LaFortune JHW, Cummings H, Liu LL, Andrews R, Stephan DW. Organometallics, 2018, 37: 4540-4544

94 Wang H, Jui NT. J Am Chem Soc, 2018, 140: 163-166

95 Discekici EH, Treat NJ, Poelma SO, Mattson KM, Hudson ZM, Luo Y, Hawker CJ, Read de Alaniz J. Chem Commun, 2015, 51: 1170511708

96 Pearson RM, Lim CH, McCarthy BG, Musgrave CB, Miyake GM. $J$ Am Chem Soc, 2016, 138: 11399-11407

97 Fuchibe K, Hatta H, Oh K, Oki R, Ichikawa J. Angew Chem Int Ed, 2017, 56: 5890-5893

98 Nishimine T, Fukushi K, Shibata N, Taira H, Tokunaga E, Yamano 
A, Shiro M, Shibata N. Angew Chem Int Ed, 2014, 53: 517-520

99 Nishimine T, Taira H, Tokunaga E, Shiro M, Shibata N. Angew Chem Int Ed, 2016, 55: 359-363

100 Okusu S, Okazaki H, Tokunaga E, Soloshonok VA, Shibata N. Angew Chem Int Ed, 2016, 55: 6744-6748

101 Nishimine T, Taira H, Mori S, Matsubara O, Tokunaga E, Akiyama H, Soloshonok VA, Shibata N. Chem Commun, 2017, 53: 1128-1131

102 Ichikawa J, Yokota M, Kudo T, Umezaki S. Angew Chem Int Ed, 2008, 47: 4870-4873

103 Fuchibe K, Jyono H, Fujiwara M, Kudo T, Yokota M, Ichikawa J. Chem Eur J, 2011, 17: 12175-12185

104 Fuchibe K, Mayumi Y, Zhao N, Watanabe S, Yokota M, Ichikawa J. Angew Chem Int Ed, 2013, 52: 7825-7828

105 Suzuki N, Fujita T, Ichikawa J. Org Lett, 2015, 17: 4984-4987

106 Fuchibe K, Imaoka H, Ichikawa J. Chem Asian J, 2017, 12: 23592363

107 Wang J, Ogawa Y, Shibata N. iScience, 2019, 17: 132-143

108 Wang J, Ogawa Y, Shibata N. Sci Rep, 2019, 9: 1-9

109 Jaiswal AK, Goh KKK, Sung S, Young RD. Org Lett, 2017, 19: 1934-1937

110 Fujimoto H, Kodama T, Yamanaka M, Tobisu M. J Am Chem Soc, 2020, 142: 17323-17328

111 Fuchibe K, Fushihara T, Ichikawa J. Org Lett, 2020, 22: 2201-2205

112 Parasram M, Gevorgyan V. Acc Chem Res, 2017, 50: 2038-2053

113 Franz AK, Wilson SO. J Med Chem, 2013, 56: 388-405

114 Patai S, Rappoport Z. The Chemistry of Organic Silicon Compounds. New York: Wiley, 2000

115 Jana A, Samuel PP, Tavcar G, Roesky HW, Schulzke C. J Am Chem Soc, 2010, 132: 10164-10170

116 Azhakar R, Roesky HW, Wolf H, Stalke D. Chem Commun, 2013, 49: $1841-1843$

117 Mallick S, Xu P, Würthwein EU, Studer A. Angew Chem Int Ed, 2019, 58: 283-287

118 Liu XW, Zarate C, Martin R. Angew Chem Int Ed, 2019, 58: 2064 2068

119 Coates G, Tan HY, Kalff C, White AJP, Crimmin MR. Angew Chem Int $E d, 2019,58: 12514-12518$

120 Evano G, Blanchard N, Toumi M. Chem Rev, 2008, 108: 3054-3131

121 Hartwig JF. Nature, 2008, 455: 314-322

122 Ma D, Cai Q. Acc Chem Res, 2008, 41: 1450-1460

123 Cho SH, Kim JY, Kwak J, Chang S. Chem Soc Rev, 2011, 40: 50685083

124 Louillat ML, Patureau FW. Chem Soc Rev, 2014, 43: 901-910

125 Bariwal J, Van der Eycken E. Chem Soc Rev, 2013, 42: 9283-9330

126 Hartwig JF. Acc Chem Res, 1998, 31: 852-860

127 Wolfe JP, Wagaw S, Marcoux JF, Buchwald SL. Acc Chem Res, 1998, 31: 805-818

128 Beletskaya IP, Cheprakov AV. Coord Chem Rev, 2004, 248: 2337 2364

129 Hartwig JF. Acc Chem Res, 2008, 41: 1534-1544

130 Brown DG, Boström J. J Med Chem, 2016, 59: 4443-4458

131 Boström J, Brown DG, Young RJ, Keserü GM. Nat Rev Drug Discov, 2018, 17: 709-727

132 MacDonald AA, DeWitt SH, Hogan EM, Ramage R. Tetrahedron Lett, 1996, 37: 4815-4818

133 Seto M, Aramaki Y, Okawa T, Miyamoto N, Aikawa K, Kanzaki N, Niwa SI, Iizawa Y, Baba M, Shiraishi M. Chem Pharm Bull, 2004 , 52: $577-590$

134 Ikemoto T, Ito T, Nishiguchi A, Miura S, Tomimatsu K. Org Process Res Dev, 2005, 9: 168-173

135 Choi DR, Shin JH, Yang J, Yoon SH, Jung YH. Bioorg Med Chem Lett, 2004, 14: 1273-1277

136 Koga H, Itoh A, Murayama S, Suzue S, Irikura T. J Med Chem, 1980, 23: 1358-1363

137 Tian J, Hustad PD, Coates GW. J Am Chem Soc, 2001, 123: 5134 5135

138 Arriola DJ, Carnahan EM, Hustad PD, Kuhlman RL, Wenzel TT.
Science, 2006, 312: 714-719

139 Pasumansky L, Hernández AR, Gamsey S, Goralski CT, Singaram B. Tetrahedron Lett, 2004, 45: 6417-6420

140 Bole LJ, Davin L, Kennedy AR, McLellan R, Hevia E. Chem Commun, 2019, 55: 4339-4342

141 Chen J, Huang D, Ding Y. Eur J Org Chem, 2017, 2017: 4300-4304

142 Wang T, Magnin DR, Hamann LG. Org Lett, 2003, 5: 897-900

143 Puthiaraj P, Pitchumani K. Chem Eur J, 2014, 20: 8761-8770

144 Liu D, Luo Y, Gao W, Cui D. Organometallics, 2010, 29: 1916-1923

145 Fontaine PP, Ueligger S, Klosin J, Hazari A, Daller J, Hou J. Organometallics, 2015, 34: 1354-1363

146 Fontaine PP, Klosin J, McDougal NT. Organometallics, 2012, 31: 6244-6251

147 Chen J, Huang D, Ding Y. Tetrahedron Lett, 2017, 58: 4240-4242

148 Thomas S, Collins CJ, Cuzens JR, Spiciarich D, Goralski CT, Singaram B. J Org Chem, 2001, 66: 1999-2004

149 Lin Y, Li M, Ji X, Wu J, Cao S. Tetrahedron, 2017, 73: 1466-1472

150 Jacobsen CB, Meldal M, Diness F. Chem Eur J, 2017, 23: 846-851

151 Nie J, Guo HC, Cahard D, Ma JA. Chem Rev, 2011, 111: 455-529

152 Kühnel MF, Lentz D. Angew Chem Int Ed, 2010, 49: 2933-2936

153 Fuchibe K, Ohshima Y, Mitomi K, Akiyama T. Org Lett, 2007, 9: 1497-1499

154 Melero C, Herrera R, Guijarro A, Yus M. Chem Eur J, 2007, 13: 10096-10107

155 Ooi T, Uraguchi D, Kagashima N, Maruoka K. Tetrahedron Lett, 1997, 38: 5679-5682

156 Kong X, Zhang H, Xiao Y, Cao C, Shi Y, Pang G. RSC Adv, 2015, 5: $7035-7048$

157 Meng X, Cai Z, Xiao S, Zhou W. J Fluorine Chem, 2013, 146: 70-75

158 Engelhart JU, Lindner BD, Tverskoy O, Rominger F, Bunz UHF. $J$ Org Chem, 2013, 78: 10832-10839

159 Bizier NP, Wackerly JW, Braunstein ED, Zhang M, Nodder ST, Carlin SM, Katz JL. J Org Chem, 2013, 78: 5987-5998

160 Diness F, Fairlie DP. Angew Chem Int Ed, 2012, 51: 8012-8016

161 Huang H, Lambert TH. Angew Chem Int Ed, 2020, 59: 658-662

162 Kamlet MJ, Abboud JLM, Abraham MH, Taft RW. J Org Chem, 1983, 48: 2877-2887

163 Champagne PA, Pomarole J, Thérien MÈ, Benhassine Y, Beaulieu S, Legault CY, Paquin JF. Org Lett, 2013, 15: 2210-2213

164 Champagne PA, Saint-Martin A, Drouin M, Paquin JF. Beilstein J Org Chem, 2013, 9: 2451-2456

165 Champagne PA, Drouin M, Legault CY, Audubert C, Paquin JF. $J$ Fluorine Chem, 2015, 171: 113-119

166 Keddie NS, Champagne PA, Desroches J, Paquin JF, O'Hagan D. Beilstein J Org Chem, 2018, 14: 106-113

167 Ma X, Mai S, Zhou Y, Cheng GJ, Song Q. Chem Commun, 2018, 54: 8960-8963

168 Deng S, Chen H, Ma X, Zhou Y, Yang K, Lan Y, Song Q. Chem Sci, 2019, 10: 6828-6833

169 Ma X, Zhou Y, Song Q. Org Lett, 2018, 20: 4777-4781

170 Yu X, Zhou Y, Ma X, Song Q. Chem Commun, 2019, 55: 8079-8082

171 Ma X, Deng S, Song Q. Org Chem Front, 2018, 5: 3505-3509

172 Ma X, Su J, Zhang X, Song Q. iScience, 2019, 19: 1-13

173 Su J, Ma X, Ou Z, Song Q. ACS Cent Sci, 2020, 6: 1819-1826

174 Ma X, Yu X, Huang H, Zhou Y, Song Q. Org Lett, 2020, 22: 52845288

175 Dryzhakov M, Richmond E, Li G, Moran J. J Fluorine Chem, 2017, 193: 45-51

176 Terao J, Begum SA, Shinohara Y, Tomita M, Naitoh Y, Kambe N. Chem Commun, 2007, 855-857

177 Sunassee SN, Davies-Coleman MT. Nat Prod Rep, 2012, 29: 513535

178 Ham SW, Choe JI, Wang MF, Peyregne V, Carr BI. Bioorg Med Chem Lett, 2004, 14: 4103-4105

179 Zhivetyeva SI, Selivanova GA, Goryunov LI, Bagryanskaya IY, Shteingarts VD. J Fluorine Chem, 2015, 180: 21-32

180 Ma X, Xuan Q, Song Q. Acta Chim Sin, 2018, 76: 972-976 
181 Yu C, Su J, Ma X, Zhou Y, Song Q. Asian J Org Chem, 2019, 8: 694-697

182 Ma X, Huang H, Su J, Song Z, Nakano T, Song Q. Chin J Chem, 2020, 38: 63-68

183 Ma X, Song Q. Chem Soc Rev, 2020, 49: 9197-9219

184 Haufe G, Suzuki S, Yasui H, Terada C, Kitayama T, Shiro M, Shibata N. Angew Chem Int Ed, 2012, 51: 12275-12279

185 Yang K, Song Q. Org Lett, 2016, 18: 5460-5463

186 Yang K, Song Q. Green Chem, 2016, 18: 932-936

187 Yang K, Zhang G, Song Q. Chem Sci, 2018, 9: 7666-7672

188 Kuang Z, Yang K, Zhou Y, Song Q. Chem Commun, 2020, 56: 64696479

189 Xia PJ, Ye ZP, Hu YZ, Xiao JA, Chen K, Xiang HY, Chen XQ, Yang H. Org Lett, 2020, 22: 1742-1747

190 Mfuh AM, Doyle JD, Chhetri B, Arman HD, Larionov OV. J Am Chem Soc, 2016, 138: 2985-2988

191 Chen K, Cheung MS, Lin Z, Li P. Org Chem Front, 2016, 3: 875879

192 Xu W, Jiang H, Leng J, Ong HW, Wu J. Angew Chem Int Ed, 2020, 59: 4009-4016

193 Xia PJ, Song D, Ye ZP, Hu YZ, Xiao JA, Xiang HY, Chen XQ, Yang H. Angew Chem Int Ed, 2020, 59: 6706-6710

194 Takahashi K, Shimoi M, Watanabe T, Maeda K, Geib SJ, Curran DP, Taniguchi T. Org Lett, 2020, 22: 2054-2059
195 Landmann J, Hennig PT, Ignat'ev NV, Finze M. Chem Sci, 2017, 8: 5962-5968

196 Yang K, Zhang F, Fang T, Zhang G, Song Q. Angew Chem Int Ed, 2019, 58: 13421-13426

197 Gao G, Yan J, Yang K, Chen F, Song Q. Green Chem, 2017, 19: $3997-4001$

198 Su Y, Huan Do DC, Li Y, Kinjo R. J Am Chem Soc, 2019, 141: 13729-13733

199 You Z, Higashida K, Iwai T, Sawamura M. Angew Chem Int Ed, 2021, 60: 5778-5782

200 Mandal D, Gupta R, Young RD. J Am Chem Soc, 2018, 140: 1068210686

201 Mandal D, Gupta R, Jaiswal AK, Young RD. J Am Chem Soc, 2020, 142: $2572-2578$

202 Wang J, Tanaka J, Tokunaga E, Shibata N. Asian J Org Chem, 2019, 8: 641-645

203 Luo C, Bandar JS. J Am Chem Soc, 2018, 140: 3547-3550

204 Zeng H, Zhu C, Jiang H. Org Lett, 2019, 21: 1130-1133

205 Liu Z, Ouyang K, Yang N. Org Biomol Chem, 2018, 16: 988992

206 Goh KKK, Sinha A, Fraser C, Young RD. RSC Adv, 2016, 6: 4270842712

207 Idogawa R, Kim Y, Shimomori K, Hosoya T, Yoshida S. Org Lett, 2020, 22: 9292-9297 